\title{
Neural Mechanisms and Psychology of Psychedelic Ego Dissolution
}

\author{
Devon Stoliker ${ }^{1}$, Gary F. Egan ${ }^{1,2}$, Karl J. Friston ${ }^{3}$, Adeel Razi ${ }^{1,2,3,4}$ \\ ${ }^{1}$ Turner Institute for Brain and Mental Health, Monash University, Clayton, VIC \\ ${ }^{2}$ Monash Biomedical Imaging, Monash University, Clayton, VIC \\ ${ }^{3}$ Wellcome Centre for Human Neuroimaging, UCL, London, United Kingdom \\ ${ }^{4}$ CIFAR Azrieli Global Scholar, CIFAR, Toronto, Canada \\ Corresponding author: \\ Devon Stoliker (devon.stoliker@monash.edu)
}

\begin{abstract}
Neuroimaging studies of psychedelics have advanced our understanding of hierarchical brain organisation and the mechanisms underlying their subjective and therapeutic effects. The primary mechanism of action of classic psychedelics is binding to serotonergic 5HT2A receptors. Agonist activity at these receptors leads to neuromodulatory changes in synaptic efficacy that can have a profound effect on hierarchical message passing in the brain. Here, we review the cognitive and neuroimaging evidence for the effects of psychedelics; in particular, their influence on selfhood and subject-object boundaries-known as ego dissolution-surmised to underwrite their subjective and therapeutic effects. Agonism of $5 \mathrm{HT} 2 \mathrm{~A}$ receptors, located at the apex of the cortical hierarchy, may have a particularly powerful effect on sentience and consciousness. These effects can endure well after the pharmacological half-life, suggesting that psychedelics may have long-term effects on neural plasticity that may play a role in their therapeutic efficacy. Psychologically, this may be accompanied by a surrender of ego resistance that increases the repertoire of perceptual hypotheses, including those that undergird selfhood. We consider the interaction between serotonergic neuromodulation and sentience through the lens of hierarchical predictive coding, which speaks to the value of psychedelics in understanding how we make sense of the world-and specific predictions about effective connectivity in cortical hierarchies that can be tested using functional neuroimaging.
\end{abstract}

Keywords: ego dissolution; resistance; brain connectivity; 5HT2A receptors; psychedelics; psilocybin; LSD; free energy principle; metacognition; meditation; consciousness; predictive coding; hierarchy; sentience. 


\section{Table of Contents}

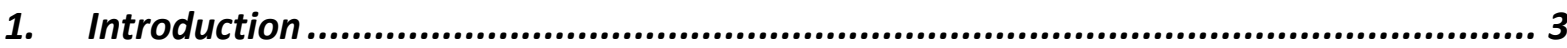

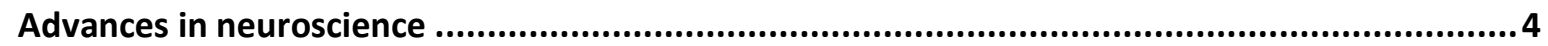

Importance of brain connectivity for subjective experience .......................................................5

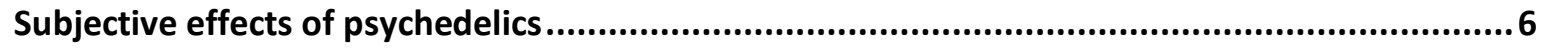

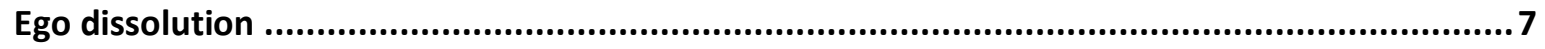

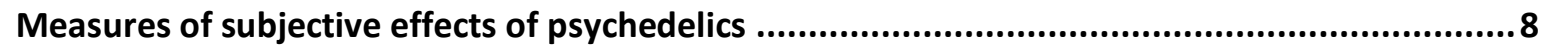

Psychedelic subjective experience and therapeutic outcomes ..................................................9

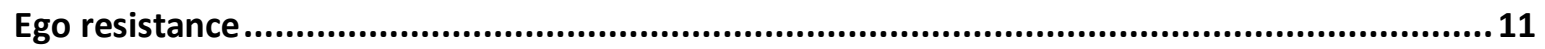

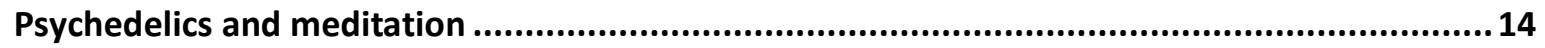

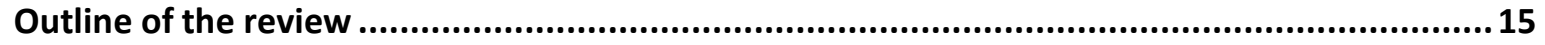

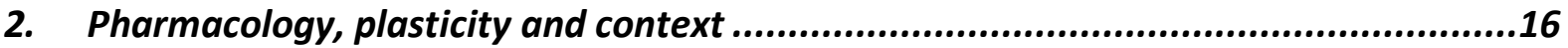

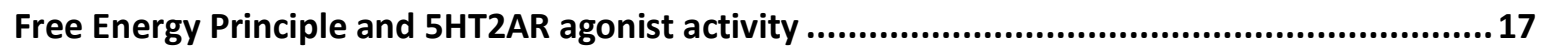

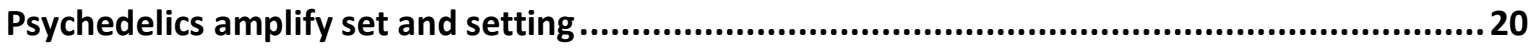

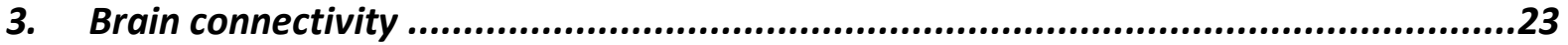

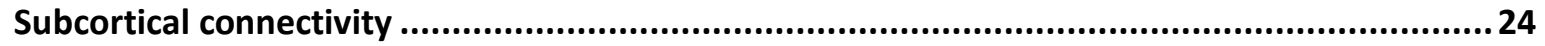

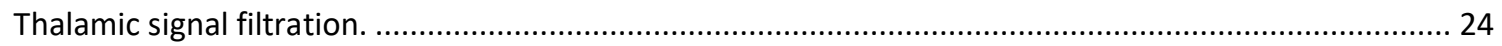

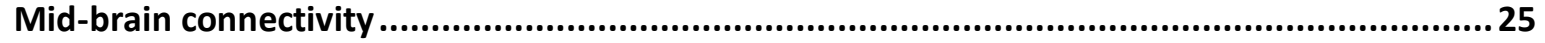

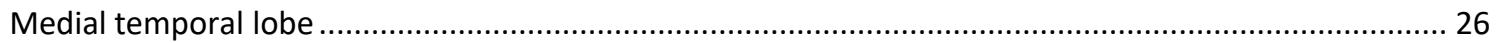

Connectivity within large-scale resting state networks ........................................................32

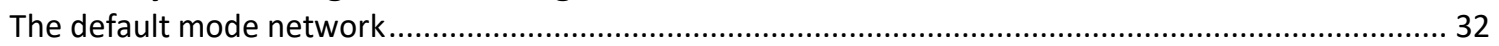

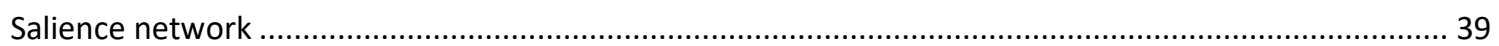

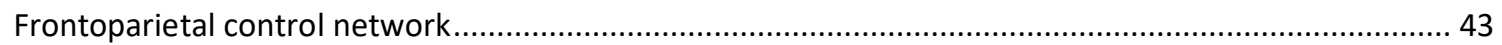

Connectivity between large-scale resting-state networks .......................................................46

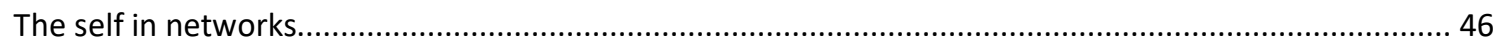

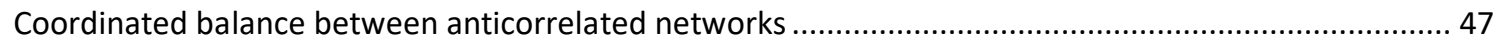

4. Cautions and methodological considerations ................................................50

Conflicts within psychedelics findings ................................................................................50

Conflicts between psychedelic findings and non-psychedelic findings....................................52

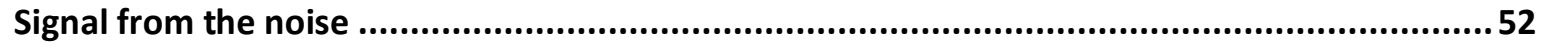

5. Unifying neuroimaging evidence of psychedelic mechanisms ...............................54

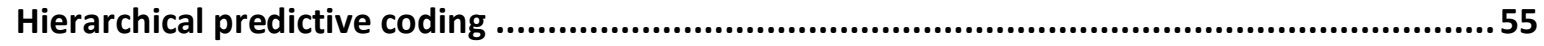

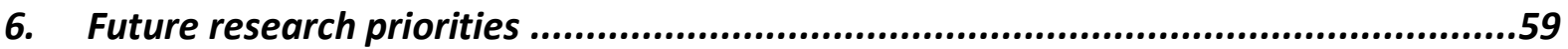

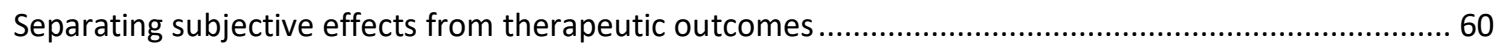

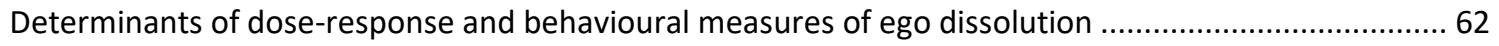

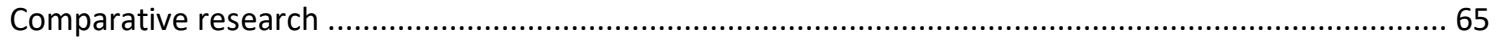

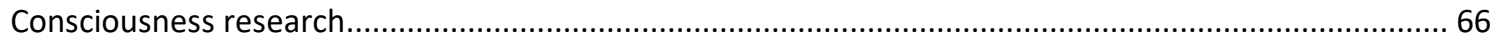

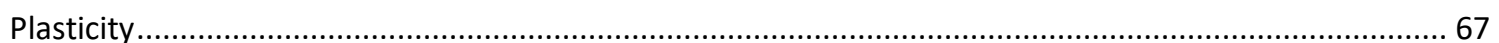

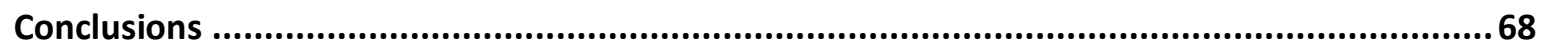




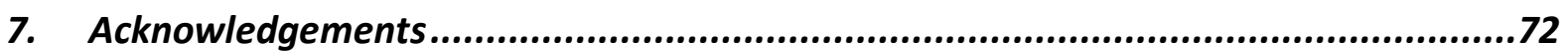

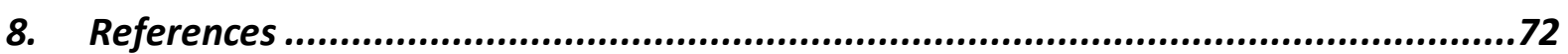

\section{Introduction}

Psychedelics constitute a class of drugs with origins in certain plants and fungi that have a long history of human use. Records indicate that their consumption in ancient cultural ritualsto achieve altered states of consciousness and spiritual insight-date back millennia (Byock, 2018; Carod-Artal, 2015; Wasson, 1978). Their inception into mainstream Western culture in the 1960s followed the synthesis of a novel psychedelic, lysergic acid diethylamide (LSD) (Nichols, 2016). This compound, which mimics the activity of its naturally derived counterparts, attracted the interest of scientists and clinical researchers who perceived similarities between the effects of psychedelics and psychosis, and considered their potential therapeutic applications (Swanson, 2018). Meanwhile, unregulated mainstream use of psychedelics, particularly LSD, challenged indoctrinated societal values and political agendas, leading to restrictions on psychedelic research by regulatory institutions (Dyck, 2005). Shortly after, psychedelics were designated by the US government, then globally, as illicit substancespossessing no medicinal or therapeutic value-and psychedelic research ground to a halt (Nichols, 2016). However, interest in the neurobiological basis of psychosis continued, and reference to early psychedelic research led to studies in healthy adults before the turn of the millennium, see (Hermle et al., 1992; Strassman, Qualls, Uhlenhuth, \& Kellner, 1994; F. X. Vollenweider et al., 1997).

In the new millennium, restrictions on psychedelic research proceeded to relax. Experimentation in the US on healthy adults resumed, showing that psychedelics can produce lasting changes in a personal sense of meaning and spiritual significance (R. R. Griffiths, Richards, McCann, \& Jesse, 2006), a notion resonant with their ancient use for spiritual guidance. In the past decade, there have been further developments in psychedelic research, 
in the setting of psychiatry. For example, the application of psychedelics to cancer-related distress marked a crucial milestone, showing that psychedelics could produce a clinically significant reduction in depression and anxiety, where alternative treatments failed (Roland R. Griffiths et al., 2016).

\section{Advances in neuroscience}

Meanwhile, neuroscience advanced considerably over the period of restricted psychedelic research. Techniques such as positron emission tomography (PET)-developed in the late 1970s, and functional magnetic resonance imaging (fMRI) in the 1990s-improved our understanding of the molecular and physiological processes in the central nervous system (Cowan, Harter, \& Kandel, 2000). The predominant conceptualisation of functional neuroanatomy at the time characterised structure-function relationships within the brain in terms of segregated areas of brain activation; namely, the principle of functional segregation (S Zeki, 2005 ). Subsequently, neuroimaging addressed the coordination of distributed neuronal responses-among functionally segregated regions-in terms of functional integration (K. J. Friston, 2011; Razi \& Friston, 2016; S Zeki \& Shipp, 1988).

Our current understanding of network activity in the brain is informed, at least in part, by the identification of resting-state brain networks; namely, intrinsic networks that remain active in the absence of goal-directed attention (Raichle, 2015). Early research, in the resting state, found that the metabolic consumption of the brain at rest requires $20 \%$ of the body's energy, while brain consumption during tasks increased this value a mere 5\% (Fox \& Raichle, 2007). Findings of this sort led to the notion that resting state activity may be an important measure of functional integration and led to the eventual identification of multiple resting state networks. Their role in mentation was confirmed by research showing that activity in resting state networks could predict cognitive performance (Arieli, Sterkin, Grinvald, \& Aertsen, 1996; Tavor 
et al., 2016; Tsodyks, Kenet, Grinvald, \& Arieli, 1999) and identify psychopathology (Fox \& Raichle, 2007).

The reintroduction of psychedelics to scientific research offers neuroscience new opportunities to use brain imaging to further our understanding of neurological processes underlying brain function and mental health. Evidence for therapeutic outcomes, following psychedelic treatment, suggests that therapeutic outcomes will be accompanied by measurable changes in brain connectivity. Moreover, measurement of acute changes in connectivity under psychedelics may offer an opportunity to better understand the neural correlates of sentience and consciousness.

\section{Importance of brain connectivity for subjective experience}

The fundamental importance of brain connectivity for perception may appear obvious, however a close analysis serves as a reminder that objective reality is quintessentially subjective. This is demonstrated by brain disorders and injuries that impair perception such as face perception, known as prosopagnosia (Wolland \& Hagelsteen, 1991), and personality, described in the famous case of Phineas Gage (O'Driscoll \& Leach, 1998). Damage to the brain can also alter representations of space and time (Buzsáki \& Llinás, 2017) with dramatic consequences for perception, such as in cases of akinetopsia (S. Zeki, 1991). Disorders such as schizophrenia and psychosis provide complementary evidence, suggesting that altered patterns of brain connectivity can affect perception and selfhood (Griffa et al., 2019; Li et al., 2019). Research has also identified brain regions and connectivity underlying altered subjective experience; including out of body experiences (Blanke \& Arzy, 2005), spiritual experiences (Miller et al., 2019), experiences of self-transcendence (Urgesi, Aglioti, Skrap, \& Fabbro, 2010), self-awareness (Ham et al., 2014) and self-reference (Bréchet, Grivaz, Gauthier, \& Blanke, 2018). These examples provide a strong parallel to the subjective effects of psychedelics. 


\section{Subjective effects of psychedelics}

Classic psychedelics are a group of perception-altering drugs classified as entheogens (Ruck, Bigwood, Staples, Ott, \& Wasson, 1979). Entheogens are substances that, when ingested, manifest an altered state of consciousness considered to have spiritual or existential significance (Winkelman \& Hoffman, 2015). This differentiates them from related empathogen drugs such as 3,4-methylenedioxymethamphetamine (MDMA, commonly known as Ecstasy) known to induce effects related to empathy, such as emotional warmth and connectedness (Bedi, Hyman, \& de Wit, 2010; Byock, 2018; Wagner et al., 2017).

Classic psychedelics exist in multiple forms, both from natural sources and chemically synthesised. The most widely recognised of these include psilocybin, mescaline, N,Ndimethyltryptamine (DMT) and LSD. Psilocybin, considered the prototypical psychedelic, is the prodrug of the psychoactive compound psilocin that occurs naturally in the psychoactive Psilocybe and related genera of mushrooms, known colloquially as magic mushrooms. Like DMT, the psychoactive ingredient in the psychedelic brew, ayahuasca, and mescaline, found in some species of cacti, psilocybin has a long history of use in some human cultural contexts (Nichols, 2016). The contemporary counterpart to plant and fungi-based psychedelics, LSD, which is technically semi-synthetic ${ }^{1}$, has been of primary interest in modern psychedelic culture and research. These entheogenic drugs share the capacity to alter the sense of identity and perspective in addition to causing hallucinations at sufficient doses (Swanson, 2018). Cognitive and metacognitive effects include shifts in attention and perspective, reframing of the relationship of self with the world and an altered perception of time and space, sense of transcendence, clarity and insight, positive emotional state and an increased sense of unity (Byock, 2018; Alexander V. Lebedev et al., 2015; K. H. Preller et al., 2015; Tagliazucchi, Carhart-Harris, Leech, Nutt, \& Chialvo, 2014; Zamaria, 2016). Psychedelic experiences have

\footnotetext{
${ }^{1}$ LSD-25 (LysergSäure-Diethylamid) was the $25^{\text {th }}$ compound synthesised by Albert Hofmann in 1938 in a drug discovery program, and its psychedelic effects were later accidentally discovered by Hofmann in 1943.
} 
been characterised as mystical-type experiences (R. R. Griffiths et al., 2006) and peak experiences (Cummins \& Lyke, 2013). However, no characterisation subsumes and epitomises the psychedelic experience more precisely than the fundamental alteration of self, known as ego dissolution.

\section{Ego dissolution}

Ego dissolution is the definitive quality of the subjective experience resulting from ingesting a sufficient dose of a classic psychedelic. The term ego dissolution emerged in the mid-20th century to denote the effects of LSD and mescaline (Millière, 2017) and has been variously described as: the disintegration of boundaries between one's self and the world; a breakdown of one's sense of self (Lebedev et al., 2015); cognition unbounded by models of the self (Letheby \& Gerrans, 2017); a compromised sense of an integrated and distinct identity (Tagliazucchi et al., 2016); and antithetical to self-assuredness (Nour, Evans, Nutt, \& CarhartHarris, 2016). Stanislav Grof-well known for his early studies of LSD and its effects on the psyche-similarly expressed ego dissolution as "an ecstatic-state, characterised by the loss of boundaries between the subjective and the objective world, with ensuing feelings of unity with other people, nature, the entire Universe, and God" (Grof, 1980). Despite its high level of abstraction, ego dissolution has been validated as an independent and measurable construct (Nour et al., 2016), with evidence suggesting that therapeutic outcomes are tied to its occurrence (R. L. Carhart-Harris \& Goodwin, 2017; Garcia-Romeu, Griffiths, \& Johnson, 2014; Roseman, Nutt, \& Carhart-Harris, 2018; Yaden \& Griffiths, 2020) although this is debated (R. L. Carhart-Harris \& Goodwin, 2017; Olson, 2020).

Ego dissolution overlaps with other descriptions of psychedelic experience, such as the aforementioned mystical-type experiences (R. R. Griffiths et al., 2006) or peak experiences (Cummins \& Lyke, 2013) and remains somewhat confounded with other facets of the psychedelic experience, such as the unitive state, which is described as a sense of oneness (R. L. Carhart-Harris, Erritzoe, Haijen, Kaelen, \& Watts, 2018; Nour, Evans, Nutt, \& Carhart- 
Harris, 2016). The early onset of ego dissolution and overlap with peak experiences suggest it is a prerequisite to access the range and richness of psychedelic experiences. Interestingly, ego dissolution is thought to be a binary state-an all or nothing phenomenon (R. L. CarhartHarris \& Friston, 2019), suggesting a dose-response related threshold that, once surpassed, realises a phenomenological shift in consciousness. PET research endorses the notion of a nonlinear dose-response relationship, through investigation of plasma psilocin (the metabolite of psilocybin) occupancy in the brain and reported subjective intensity of experience (Madsen et al., 2019). However, the ego dissolution dose-response relationship is relatively unexplored. It is influenced by external stimuli, particularly music (Kaelen et al., 2018) and some psychedelics, such as psilocybin, are reported to produce ego dissolution more reliably than others, such as LSD (Liechti, 2017). Following the onset of ego dissolution, psychedelic experiences are thought to catalyse spiritual and personal insight through introspection (Letheby \& Gerrans, 2017; Malone et al., 2018; (R. G. Dos Santos \& Hallak, 2020). The ensuing insights can result in lasting therapeutic effects and transformation of self-related beliefs (Lyons \& Carhart- Harris, 2018).

\section{Measures of subjective effects of psychedelics}

Acute subjective effects of psychedelics have been measured in experimental studies using the Hallucinogen Rating Scale (HRS) (Strassman et al., 1994), the Mystical Experiences Questionnaire (MEQ) (Maclean, Leoutsakos, Johnson, \& Griffiths, 2012) and the Abnormal Mental States (APZ) questionnaire, renamed the Altered States of Consciousness Rating Scale (OAV) (Studerus, Gamma, \& Vollenweider, 2010) and revised as the 5 Dimensions of Altered States of Consciousness scale (5D-ASC) (Dittrich, 1998). This scale includes three primary dimensions: oceanic boundlessness (OBN) or positively felt ego dissolution, dread of ego dissolution (DED) or negatively felt ego dissolution, and visionary restructuralisation (VRS) or visual alterations and altered meaning of precepts. The two other (secondary) dimensions are referred to as auditory alterations (AA) and a vigilance state (VIR). 
Subsequent psychometric evaluation of the OAV/5D-ASC revealed eleven factors that correlated with the original OAV scales: spiritual experience, changed meaning of percept, insightfulness, anxiety, impaired control and cognition, experience of unity, complex imagery, elementary imagery, blissful state, audio-visual synaesthesia, and disembodiment (Nichols, 2016; Studerus et al., 2010). A more recent (and less widely explored) inventory-that validates ego dissolution as an independent construct-focuses on the phenomenon of ego dissolution and is aptly named the Ego Dissolution Inventory (EDI) (Nour et al., 2016). While the precise phenomenological classification or taxonomy of the subjective effects of psychedelic drugs are incomplete, the factors identified in the HPS and 5D-ASC provide formal deconstructions of the subjective experience under classic psychedelics. Ego dissolution, however, as exemplified by the development of the EDI and emphasis in the 5DASC, is perhaps the best characterisation of the primary subjective effect of psychedelics.

\section{Psychedelic subjective experience and therapeutic outcomes}

Interest in psychedelics has been renewed in part due to their tolerability (Rafael G. dos Santos, Carlos Bouso, Angel Alcazar-Corcoles, \& Hallak, 2018), safety (Studerus et al., 2010), and the provision of guidelines for safe administration (M. Johnson, Richards, \& Griffiths, 2008). This interest includes considering psychedelics as an adjunct to psychotherapy. Unlike traditional psychotherapy, which relies primarily on interpersonal discourse, psychedelic treatment relies on an introspective, endogenous, experience as the patient typically lays silent, blindfolded and in a state of rest (Nutt \& Carhart-Harris, 2020). After the therapeutic session, patients are usually invited to a more traditional psychotherapeutic session coined integration, in which the patient and therapist, often a psychiatrist or psychologist discuss the experience in the hope of deriving insights that may help cement positive life changes. Under supportive conditions, changes to patterns of thought and behaviour-underlying psychiatric conditions such as anxiety, addiction and depression—can show significant improvement from 
as little as one or just a few psychedelic sessions (Griffiths et al., 2011; Nichols et al., 2016; (Letheby \& Gerrans, 2017a).

The transformative experience psychedelics produce offers a therapeutic tool that differs from traditional pharmacotherapy, which relies on neurochemical mechanisms to produce therapeutic effects. Although there is some evidence that certain psychiatric conditions, such as obsessive compulsive disorder (OCD) (Majic, Schmidt, \& Gallinat, 2015), may respond more to the physiological effects of psychedelics, the clinical literature suggests that the psychological experience elicited by psychedelics is integral to transformative therapeutic change (Nutt \& Carhart-Harris, 2020; Roseman, Nutt, et al., 2018; Yaden \& Griffiths, 2020). This is reflected in the dependence of lasting therapeutic outcomes upon the spiritual or personally meaningful quality of ego dissolution, here read as mystical experiences (Bogenschutz et al., 2015; R. L. Carhart-Harris, Bolstridge, et al., 2016; Garcia-Romeu et al., 2014; Roland R. Griffiths et al., 2016; Roland R. Griffiths et al., 2011; Hartogsohn, 2018; M. W. Johnson, Garcia-Romeu, Cosimano, \& Griffiths, 2014; Roseman, Nutt, et al., 2018; Ross et al., 2016; Yaden \& Griffiths, 2020).

Transformative outcomes from the psychedelic experience can be linked to the early $19^{\text {th }}$ century psychological theory known as filtration theory. Filtration theory suggests information entering consciousness is winnowed by selection processes that filter the subconscious material (Marshall, 2005; Swanson, 2018). The term psychedelic-which originates from the

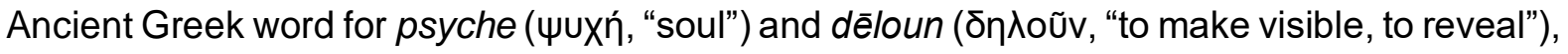
translating to "mind-manifesting"-was inspired by this theory to describe the inhibition of filtration processes in the brain, relaxing constraints on perception, emotions, thought, and sense of self (Osmond, 1957; Swanson, 2018). Under this theory, psychedelics may be considered to facilitate and synergise with the psychoanalytic process. In the patientpractitioner relationship, as in the relationship with oneself, some thoughts and feelings may be openly identified or disclosed while others are inhibited or avoided. The disinhibition of the 
normal constraints of mentation by psychedelics may allow activity-usually supressed to the subconscious and constrained by conscious processes-to surface into conscious awareness. The description of this process accords with modern accounts of therapeutic applications of psychedelics. For example, the introspective experiences of addicts treated with psychedelics featured personally relevant emotional content, otherwise inhibited from conscious awareness, to surface, and the resulting nuanced personal insight led to reduced substance-dependency (Malone et al., 2018). Similar accounts are also documented in the unmonitored use of psychedelics (Zamaria, 2016). Although the merit of insights derived under psychedelics warrants some scepticism (see Letheby 2016 (Letheby, 2016)), evidence that ego dissolution targets the most abstract levels of processing-responsible for selecting channels that provide input to conscious awareness and their capacity to produce therapeutic insight (Hartogsohn, 2018)—endorses filtration theory as a rudimentary model of the action of psychedelics that is consistent with changes in hierarchical connectivity, as we will see below.

\section{Ego resistance}

Psychedelic phenomenology has recently been described under the free energy principle (FEP). The FEP explains the innate tendency of living organisms to minimise surprise and will be explored later in detail. Elaborations of the FEP, applied to psychoanalysis, suggest the tendency to minimise surprise can lead to maladaptive beliefs that are resistant to change ( $R$. L. Carhart-Harris \& Friston, 2019) and that psychedelics may dissolve recalcitrant beliefs. Psychedelic ego dissolution appears to feature in this [therapeutic] process by removing a hurdle and relaxing psychological resistance. The mechanism of resistance-release is thought to be a central feature of ego dissolution associated with the therapeutic effects of psychedelics.

The term psychological resistance is typically used to denote psychotherapeutic exchanges in which patients avoid particular uncomfortable topics. However, resistance may be 
fundamental to mental wellbeing and is taken more broadly as the trigger of ego defences; namely, invoking beliefs and attitudes in response to perceived threats. Freudian psychoanalytic theory helps frame this process as the ego being maintained in a solved condition rather than an unsolved state (Solms, 2018). Freud described this preference as the source of resistance that elicits defence (Solms, 2018). Therefore, occurrences of resistance may be considered as the departure of the ego from the solved state. The ego, wishing to return to the solved state, may manifest self-beliefs that provide a form of emotional catharsis, or the best attempt at the solved state, in lieu of more authentic solutions, reminiscent of Freud's wish fulfilment.

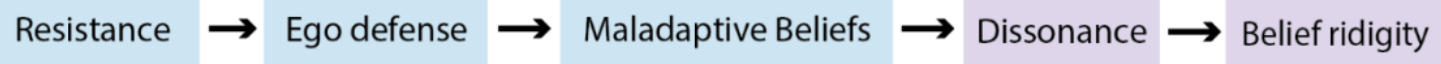

Figure 1. Elementary model of resistance, leading to rigid or inflexible beliefs. Resistance that leads to ego defense may be accompanied by rationalizations in the form of higher order beliefs. Higher order beliefs that are maladaptive may lead to further experiences of resistance that evoke dissonance between emotions and experiences, which fortify maladaptive beliefs leading to belief rigidity.

If the beliefs are maladaptive, they may lead to further experiences of resistance that produces incongruence, or dissonance, between the innate desire for positive emotional self-worth and the experiences. This incongruence or dissonance maintains the unsolved state and is similar to Festinger's cognitive dissonance that describes the discomfort of conflict between action and cognition (Festinger, 1962). Here, the concept of dissonance is extended to describe the conflict between emotional self-worth and experiences that the ego resolves through beliefs.

Maladaptive beliefs are those which fail to reduce experiences of resistance and instead may perpetuate dissonance that in turn perpetuates resistance, leading to the development of rigid, ingrained patterns of thinking, see Figure 1. In more extreme cases, these unhelpful beliefs 
may develop into symptoms of psychopathological disorders; the exact presentation of disorder may resemble how the dissonance between experiences and emotion is resolved through the cognition. In the case of depression, a bullied individual may cathartically solve dissonance between damaging experiences and the innate emotional desire for self-worth by forming beliefs about their inferiority; thereby enabling the ego to enter a proxy solved state. In the case of narcissism, dissonance between emotional self-worth and conflicting experiences may be solved through belief in the inferiority of others. Alternately a more straightforward instance of resistance may be evinced in the exaggerated emotional response to disappointment, characteristic of borderline personality disorder (Houben, Claes, Sleuwaegen, Berens, \& Vansteelandt, 2018).

Although dissonance between self-worth and experience is also underlined in this scenario, resistance can also be seen as resistance to experience (and implicitly, belief updating). In all cases, resistance leads the ego into an unsolved state that is dissonant and can form maladaptive beliefs that are characterised by further resistance. Psychedelics target this psychological ego resistance and introduce a permissive flexibility in cognition that dissolves rigid patterns of thinking and is reflective of the psychoanalytic process ${ }^{2}$. From the perspective of FEP, psychedelics dissolve precise belief states, where that dissolution can be quantified as an increase in entropy or decrease in precision (R. L. Carhart-Harris, 2018a). Technically, the precision of a (probabilistic or Bayesian) belief corresponds to its negative entropy. This dissolution has been connected to the personality trait of openness (A. V. Lebedev et al., 2016) and is arguably antithetical to resistance. The dissolving of resistance through successful ego dissolution therefore appears to be commensurate with the relaxation of precise (priors) beliefs and ego defences that enable deeply ingrained patterns of thoughts to be critically evaluated and revised in therapeutic settings (R. L. Carhart-Harris et al., 2014).

\footnotetext{
${ }^{2}$ Working through resistance in psychotherapeutic exchanges between practitioner and patient and in successful psychedelic ego dissolution experiences contributes to lasting therapeutic changes (LaFarge, 2011; Roseman, Nutt, et al., 2018).
} 


\section{Psychedelics and meditation}

The elusion of ego resistance is also elemental to the practice of non-judgemental awareness through meditation. Like the use of psychedelics, meditation is another practice rooted in spirituality, dating back millennia. Meditation is a non-pharmacological intervention with a wealth of evidence supporting positive therapeutic outcomes (Keng, Smoski, \& Robins, 2011). Meditation and psychedelics share similar processes of dissolving the self and attaining insight, and both demonstrate the capacity to inspire insights leading to personal growth and improved wellbeing (Roland R. Griffiths et al., 2018). Similarities in the brain connectivity patterns underlying states of meditation and psychedelic states have been reported, particularly in resting-state networks (Brewer et al., 2011; R. L. Carhart-Harris, Erritzoe, et al., 2012; Heuschkel \& Kuypers, 2020; Alexander V. Lebedev et al., 2015). Recent studies of psychedelics and meditation suggest the practice of meditation may be beneficial to the psychedelic experience under psilocybin by reducing anxiety (Smigielski, Kometer, et al., 2019). Similarly, psychedelics may enhance meditation and spiritual practice.

Single doses of psilocybin administered to expert meditators were found to improve depth of meditation and long-term wellbeing beyond the beneficial effects of exclusive long-term meditation practice (Smigielski, Scheidegger, Kometer, \& Vollenweider, 2019). Another study exploring psilocybin-in conjunction with (non-secular) spiritual activities-suggested their combination boosts lasting prosocial attitudes and sustains personal spiritual practice (Roland R. Griffiths et al., 2018). Further studies suggest a complementary effect of psilocybin and mindfulness meditation in the treatment of depression (Heuschkel \& Kuypers, 2020). Reference to meditation in psychedelic research is both common and valuable by helping to define similarities and differences between psychedelic phenomenology and brain connectivity. One of the most prominent differences is the phenomenological richness of the psychedelic experience; see Millière, Carhart-Harris, Roseman, Trautwein \& Berkovich- 
Ohana, 2018 for an in depth review and exploration of their potential synergy; Heuschkel \& Kuypers, 2020 for a review of meditation complementing psilocybin in the treatment of depression; and Payne, Chambers \& Liknaitzky for a review of clinical synergies (Payne, Chambers, \& Liknaitzky, 2021). The effectiveness of meditation through non-judgement and non-resistance may reflect an innate self-corrective tendency of the mind towards the reduction of psychological resistance to engender wellbeing. This tendency often fails under ordinary control of the ego and appears to be facilitated in a similar fashion by both psychedelics and meditation.

\section{Outline of the review}

A comprehensive understanding of psychedelics requires consideration of historical, cultural, sociological, spiritual, philosophical, psychological and physiological perspectives. Our focus will be on the effects of psychedelics on brain connectivity and the mediation of subjective effects, particularly ego dissolution. Psychedelic studies that used non-invasive brain imaging including fMRI, PET, electroencephalography (EEG) and magnetoencephalography (MEG) are surveyed with a special emphasis on how modelling and estimating brain connectivity may shed light on the neural mechanisms that underlie the psychedelic experience.

The remainder of this review begins with the molecular and pharmacological action of psychedelics, and their effect on cognition. The effects of psychedelics on neuroreceptors are central to formulations of their action under hierarchical models of brain connectivity. These models explain the ability of psychedelics to increase cognitive flexibility and speak to the importance of context and administration setting. Building on the pharmacological action at the neuronal level, the role of neuronal populations in subcortical structures is reviewed. In particular, the thalamus is a highly connected structure attributed with a gating function that filters information exchanged with the cortex (Torrico \& Munakomi, 2019). Its function under psychedelics is well aligned with filtration theory. Next, we consider medial temporal lobe 
(MTL) connectivity. MTL regions are involved in memory and emotion (Stein et al., 2000; Steriade \& Llinas, 1988; Voets et al., 2015) and may share an association with ego dissolution and the therapeutic engagement of emotion under psychedelics (R. L. Carhart-Harris et al., 2017; Kraehenmann et al., 2015). Moving to the cortex, altered 'resting-state' brain connectivity across distinct networks —-typically involved in abstract and executive functionsare considered. Finally, integration of these systems and unifying theories of psychedelic effects are explored before considering future research directions.

\section{Pharmacology, plasticity and context}

Classic psychedelics such as LSD, psilocybin, mescaline and DMT are characterised by agonist activity at the serotonin (5HT) 2A receptor (Robin Lester Carhart-Harris et al., 2014; (Dinis-Oliveira, 2017; Nichols, 2016). These psychedelic compounds share a common affinity for the 5HT2A receptor (5HT2AR) (Ly et al., 2018). The binding kinetics and occupancy of the 5HT2AR predict their subjective effects (Glennon, Teitler, \& Sanders-Bush, 1992; (Madsen et al., 2019). While the molecular structures of psychedelics vary ${ }^{3}$-and many act at non-5HT receptors (David E. Nichols, 2016; Tyls et al., 2014)—the importance of the 5HT2AR has been established using antagonists such as ketanserin, which blocks the receptor and inhibits subjective effects (Katrin H. Preller et al., 2018; Quednow, Kometer, Geyer, \& Vollenweider, 2012). Subjective psychedelic effects may also be precluded by the rapid desensitisation of the 5HT2AR following exposure to psychedelics (Vann Jones \& O'Kelly, 2020). The 5HT2AR is a metabotropic serotonin receptor located post-synaptically on the apical dendrites of layer 5 pyramidal neurons (E. T. Weber \& Andrade, 2010). Pyramidal neurons act as the primary output from cortical regions projecting to distal cortical and subcortical regions (Spruston, 2008). Layer 5 pyramidal neurons require agonist activity to reach an action potential threshold to communicate with other neurons (Varley, Carhart-Harris, Roseman, Menon, \& Stamatakis,

\footnotetext{
${ }^{3}$ For detailed review of neurobiology of psychedelic drugs see (Franz X. Vollenweider \& Preller, 2020).
} 
2019). Psychedelic binding to the 5HT2AR depolarises the target cell to increase the likelihood of firing (Andrade, 2011). This changes the postsynaptic sensitivity of deep pyramidal neurons and consequent neuronal message passing in cortical and subcortical hierarchies that may underwrite the effects of psychedelics on distributed neuronal activity; e.g., neuronal oscillations (Varley et al., 2019). See Figure 2 for basic pharmacology of classic psychedelics.

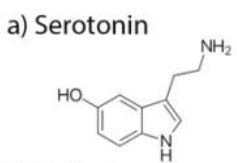

b) Psilocin

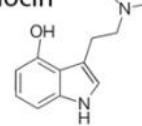

c) $5 \mathrm{HT} 2 \mathrm{~A}$ receptor

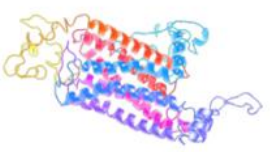

d) Neuron

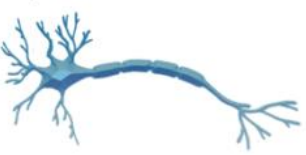

e) Canonical microcircuit

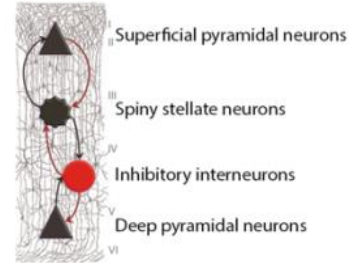

f) Brain

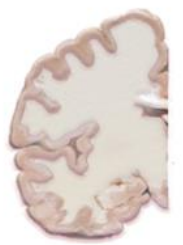

Figure 2. Basic pharmacology of classic psychedelics. Similarity between a) serotonin and classic psychedelic molecular structure, such as b) psilocybin (the prodrug of psilocin) enables binding to c) 5HT2A receptors (5HT2AR). Binding results in agonist action of the receptor, reducing the action potential threshold of d) the neuron. Specifically, psychedelics target layer 5 deep pyramidal neurons demonstrated in e) the canonical microcircuit, which is a column of the tissue that composes f) the brain and illustrates the temporal progression of forward connections.

\section{Free Energy Principle and 5HT2AR agonist activity}

Altered serotonin transmission has been linked to neuronal responses to unpredicted or surprising events (Matias, Lottem, Dugué, \& Mainen, 2017). Learning from unexpected outcomes is important for the survival of living organisms and confers a clear evolutionary advantage. The FEP provides a framework to understand responses to surprise-and the influence of psychedelics. The FEP posits that all living systems survive by minimising the difference between sensory input and predictions based upon an internal (generative) model of the world (K. Friston, 2009, 2010). This difference is described as self-information, surprisal or more simply, surprise, which can also be written as a (log) model evidence in Bayesian statistics (for which the free energy is an upper bound). This means that minimising free energy is the same as maximising model evidence, sometimes known as self-evidencing (Hohwy, 2016). 
A popular way of understanding the minimisation of free energy is in terms of minimising prediction error or, more precisely, precision weighted prediction error. Precision refers to the predictability of some random variable. This means prediction errors should only be treated as surprising if they convey precise information. In other words, a prediction error is not very useful if something is not predictable. The encoding of predictability or precision is thought to be mediated by synaptic efficacy; in other words, the sensitivity of gain of postsynaptic responses to predictions and prediction errors. Because psychedelics have a selective and profound effect on postsynaptic gain, they can be understood as rebalancing the influence of sensory evidence and prior beliefs during belief updating (mediated by neuronal message passing), in response to sensory input.

In this framework, 5HT2AR modulation by psychedelics is understood to alter the sensitivity to prediction errors at various hierarchical levels in the cortical hierarchy. In brief, psychedelics are thought to relax the precision of high-level prediction errors so that lower level (e.g., sensory) prediction errors have a greater influence. This allows multiple hypotheses and explanations for sensory input to be entertained by the brain; endowing the implicit self evidencing with a greater flexibility (R. L. Carhart-Harris, 2018a). Neurophysiological measures can express this in terms of a greater entropy or complexity of neuronal activity that underwrites belief updating.

In probability theory, entropy is a measure of uncertainty and, in some settings, complexity. Agonist activity at $5 \mathrm{HT} 2 \mathrm{AR}$ is thought to increase neuronal entropy (Bestmann et al., 2008). It serves as a metric of neuronal activity that has been used to quantify the influence of psychedelics via modulation of 5HT2AR neurotransmission (Bouso, Dos Santos, AlcazarCorcoles, \& Hallak, 2018; R. L. Carhart-Harris, 2018a; R. L. Carhart-Harris et al., 2014). Recent findings suggest that the increase in the entropy of neuronal activity is topographically organised, exhibiting increases in some brain regions and decreases in others under 
psychedelics (Herzog et al., 2020). Psychedelic induced entropy changes in the brain has also been read as a state of increased plasticity (R. L. Carhart-Harris \& Nutt, 2017; Ly et al., 2018) manifesting as cognitive flexibility (Matias et al., 2017) and an increased repertoire of possible brain states (Atasoy et al., 2017). The psychedelic enabling of neural plasticity is a key mechanism used to explain the therapeutic effects of psychedelics (Inserra, De Gregorio, \& Gobbi, 2021).

Under the FEP, psychopathology is usually interpreted in terms of false inference. For example, inferring something is present when it is not (e.g., hallucinations and delusions) or inferring something is not present when it is (e.g., agnosia and neglect syndromes). Usually, this is interpreted as inference under aberrant precision. In other words, too much or too little precision is afforded by sensory prediction errors, relative to prediction errors higher in the hierarchy that mediate prior beliefs. If prior precision is unduly high, inference about states of affairs causing sensations may proceed under inflexible priors that are maladaptive $(R$. L. Carhart-Harris \& Friston, 2019). 5HT2AR modulation under psychedelics is thought to relax overly precise prior precision and enable the revision of maladaptive priors through experience-dependent learning (e.g., of the sort that may be leveraged in psilocybin assisted psychotherapy).

The ensuing effects on 5HT2AR signalling has been suggested to mediate active coping with distress and support cognitive-emotional revision (R. L. Carhart-Harris \& Nutt, 2017). The notion of inflexible, maladaptively precise, priors is compatible with psychoanalytic theory in which rigid priors may be thought of as manifesting strong resistance. Overlap between the distributions of high-density $5 \mathrm{HT} 2 \mathrm{AR}$ and the cortical regions considered to underlie selfrelated beliefs also speaks to the notion that psychedelics may target the substrate of high order (Bayesian) beliefs (Robin Lester Carhart-Harris et al., 2014; (Beliveau et al., 2017; Komorowski et al., 2017). This formulation offers a neuronally plausible account of neuromodulation and plasticity in sentient behaviour that is supported by the FEP (R. L. 
Carhart-Harris, 2018a; Roland R. Griffiths et al., 2011; Letheby \& Gerrans, 2017a, 2017b; Tagliazucchi et al., 2014). Increased flexibility following psychedelic induced plasticity also explains the context sensitivity that individuals exhibit under psychedelics (R. L. Carhart-Harris et al., 2015).

\section{Psychedelics amplify set and setting}

Contextual factors such as states of the environment, individual traits and psychological variables are crucial in shaping the psychedelic experience (Carhart-Harris and Nutt, 2017; Robin L. Carhart-Harris et al., 2018; Carhart- Harris et al., 2014; (Tylš, Palenicek, \& Horacek, 2016) Robin L. Carhart-Harris et al., 2018; Roseman, Nutt, \& Carhart-Harris, 2018). Some early experiments with psychedelics created conditions that elicited adverse and traumatic reactions from participants (Robin L. Carhart-Harris et al., 2018). Such outcomes may have contributed to early Western characterisation of psychedelics as psychotomimetic, referring to properties that mimic psychosis (Felix Müller, Dolder, Schmidt, Liechti, \& Borgwardt, 2018). The evolving understanding of psychedelics nuanced this terminology, later describing them as hallucinogens (Nichols et al., 2016). However, neither term recognised their potential merit in therapeutic settings-leading to the evolution of the modern term psychedelic. This term encompasses the mind-manifesting nature of psychedelics as "non-specific amplifiers of consciousness" (Grof, 1980) and speaks to the importance of set and setting. This is consistent with the effect of psychedelics on the synaptic encoding of precision in terms of synaptic efficacy or postsynaptic gain, where increases in the sensitivity to prediction errors at various levels in cortical hierarchy has been associated with attentional selection. Or the instantiation of a particular attentional set (V. Ainley, Apps, Fotopoulou, \& Tsakiris, 2016; V Ainley, Tajadura-Jiménez, Fotopoulou, \& Tsakiris, 2012; Feldman \& Friston, 2010; Fotopoulou \& Tsakiris, 2017). 
Psychedelics, through 5HT2AR agonist activity, may amplify exogenous influences of the setting and endogenous influences of attentional set. The ensuing state of plasticity can be likened to feeling "lost" (e.g., in the forest) which, depending on mindset and setting, can manifest opposing (emotional) responses, see Figure 3. Recognition of the influence of set and setting has led to the development of guidelines for the safe administration of psychedelics. These include measures for screening, preparation, and supportive environmental conditions (M. Johnson et al., 2008). Under such guidelines, subjects typically respond well to even high psychedelic doses, evidenced by the large number of modern clinical trials and applications of psychedelics. To date there are no reports of serious adverse reactions (M. W. Johnson, Griffiths, Hendricks, \& Henningfield, 2018). However, even in supportive settings, challenging experiences should be anticipated. These may be viewed as part of the therapeutic self-discovery process: under the premise that challenging contents and experiences manifest from the disinhibition of ego restraints-that usually preclude therapeutically relevant emotion and cognition from entering conscious awareness (Carbonaro et al., 2016).

However, unregulated use of psychedelics remains potentially hazardous: phenomenological studies of unregulated ingestion show that users generally prepare both mindset and environmental settings before the dose intake by ensuring supportive environmental conditions generally (Zamaria, 2016). This preparation reflects the tenets of psychedelic assisted therapy and the customs of ancient ritualistic use that involve care and preparation.

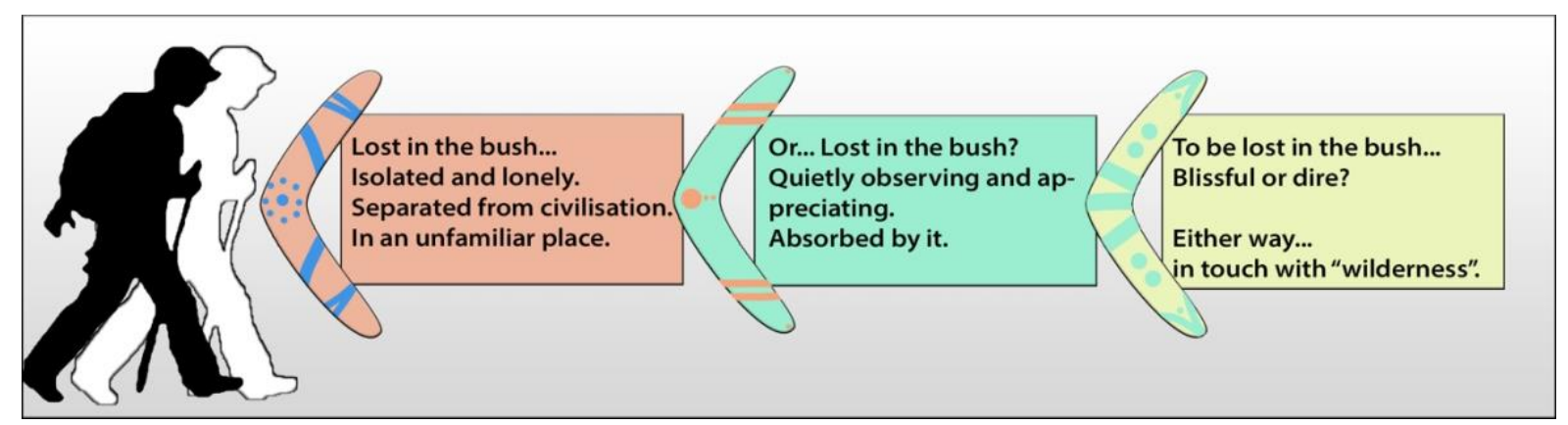


Figure 3. Lost in the bush (forest). This schematic illustrates the opposing psychological responses to psychedelic induced uncertainty dependent on context of mindset and setting. Adapted from photo taken at the rainforest gallery, Warburton, Victoria, Australia.

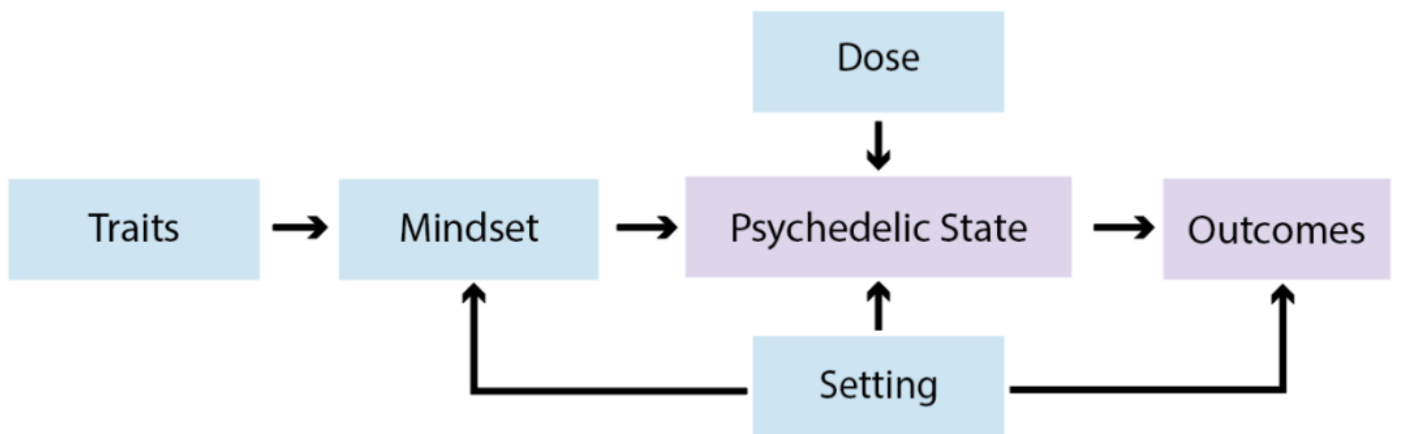

Figure 4. Extra-pharmacological model. Traits and setting influence mindset prior to administration. Mindset, setting (environment) and dosage contribute to the psychedelic experience (state) and subsequent therapeutic outcomes. Purple colour boxes represent psychedelic influenced states. Adapted from extra-pharmacological model by Carhart-Harris and Nutt, 2017.

Set and setting are crucial factors when explaining negative psychotomimetic and positive therapeutic reactions to psychedelics. Identifying the influence of set and setting is an important subfield of research: see Figure 4.

Manipulation and measurement of the impact that preparatory methods and environmental stimuli have upon psychedelic experiences has yet to be widely explored. For example, the influence of music on the psychedelic experience has been found to affect the induction of ego dissolution and insightfulness (Kaelen et al., 2018) and enhance these experiences (Frederick S. Barrett, Robbins, Smooke, Brown, \& Griffiths, 2017). Other visual manipulations-such as comparing brain activity between eyes-closed and eyes-open states-speaks to the mediation of the psychedelic experience by exogenous stimuli (Mediano et al., 2020). Furthermore, recent studies of expert meditators suggests that meditation may be an effective preparation for psychedelic experiences (Smigielski, Kometer, et al., 2019). Meditators are trained to cultivate an open, unattached and non-judgemental 
awareness of internal mentation. This practice of non-resistance and release resembles the clinical guidelines in psychedelic therapy (Wolff et al., 2020). Meditation training may reduce challenging experiences in the state of uncertainty accompanying ego dissolution and may offer a promising mode of preparation for therapeutic applications. The link between the quality of psychedelic experiences and therapeutic outcomes (Roseman, Nutt, et al., 2018) suggests that mindset preparation through meditation may be an effective means to moderate anxiety.

The evidence of contextual influences under psychedelics offers another research opportunity to explore neural plasticity and cognitive flexibility relating to 5HT2AR agonist activity. Investigating different combinations of set and setting may help direct interventions and inform clinical procedures related to set and setting. How 5HT2AR-dependent changes in distributed neuronal activity are mediated by changes in connectivity between subcortical systems and the cortex is based on insights obtained from functional neuroimaging of healthy adults, as reviewed next.

\section{Brain connectivity}

Different parts of the brain are connected to realise certain functions such as cognition, perception and decision-making. The subjective effects of psychedelics on perception suggest psychedelics may preferentially target brain regions and connections involved in associative functions related to sentience and perception. This conjecture is supported by the high densities of 5HT2AR expressed throughout associative brain regions (Pazos, Probst, \& Palacios, 1987). However, cortico-subcortical networks and limbic structures also express 5HT2AR. 5HT2AR mediated change of subcortical circuitry by psychedelics alter their functions and may account for aspects of the psychedelic subjective experience. Evidence from imaging studies can, in principle, be used to test hypotheses for the mechanism of action of psychedelics. 


\section{Subcortical connectivity}

\section{Thalamic signal filtration}

The early understanding of psychedelic mechanisms - as described in filtration theorysuggests consciousness is winnowed by selection processes that exclude subconscious material (Marshall; Swanson, 2018). The thalamus is thought to serve such a function by filtering sensory information reaching the cortex. The thalamus is an information filter and relay hub located in the centre of the brain, with projections in all directions, responsible for conveying signals between the brain and body (Torrico \& Munakomi, 2019). The thalamus is also involved in the regulation of conscious level; including sleep, wakefulness, alertness and sensorimotor arousal (Torrico \& Munakomi, 2019). It shares connectivity profiles with limbic structures (Stein et al., 2000; Steriade \& Llinas, 1988; Voets et al., 2015) and is implicated in medial temporal lobe epilepsy (Barron, Tandon, Lancaster, \& Fox, 2014) which produces psychedelic-like sensory illusions and déjà vu experiences. Disruptions to the thalamuswithin the cortico-striato-thalamo-cortical (CSTC) loop-speak to the role of the thalamus in schizophrenia (Behrendt \& Young, 2004; Halberstadt, 2015), sometimes referred to as a cognitive dysmetria. Certain portions of the thalamus (e.g., the pulvinar) have been associated with setting the precision of cortical message passing, of the sort implicated in selective attention and feature from ground separation in the visual system (R. Kanai, Y. Komura, S. Shipp, \& K. Friston, 2015).

Commonalities-between the positive symptoms of schizophrenia and hallucinations produced under psychedelics—suggest the CSTC circuitry may be involved in hallucinations and mediate increased bottom-up connectivity from the thalamus to the cortex (Geyer \& Vollenweider, 2008; Tylš et al., 2016). Examinations of functional connectivity (FC), under LSD, confirm an increase in correlations between the thalamus and cortical hubs (Felix Müller et al., 2018; Tagliazucchi et al., 2016). FC analysis under LSD also found increases in correlated activity between the thalamus and insula and fusiform gyrus, thought to be related 
to the perceptual effects of psychedelics (F. Müller et al., 2017). Functional connectivity refers to correlations between neuronal activity in different systems that is mediated by effective connectivity; namely the directed influence that one neuronal system exerts over another.

Dynamic causal modelling (DCM) for resting-state fMRI (K. J. Friston, Kahan, Biswal, \& Razi, 2014; Razi, Kahan, Rees, \& Friston, 2015; Razi et al., 2017), quantified the effective connectivity of the thalamus connectivity under LSD. The results showed increased effective connectivity from the thalamus to the posterior cingulate cortex (PCC) and decreased topdown connectivity from the PCC to the thalamus (Katrin H. Preller et al., 2019). These findings support the general notion of psychedelic enabled increase in bottom-up effects on the cortex (Franz X. Vollenweider \& Preller, 2020). This is consistent with a relaxation of prior precision in high levels of cortical hierarchies, to which the thalamus projects. In short, the thalamus appears to play a key role in enabling ascending or bottom-up connectivity under psychedelics, by opening a 'thalamic filter', see Figure 5.
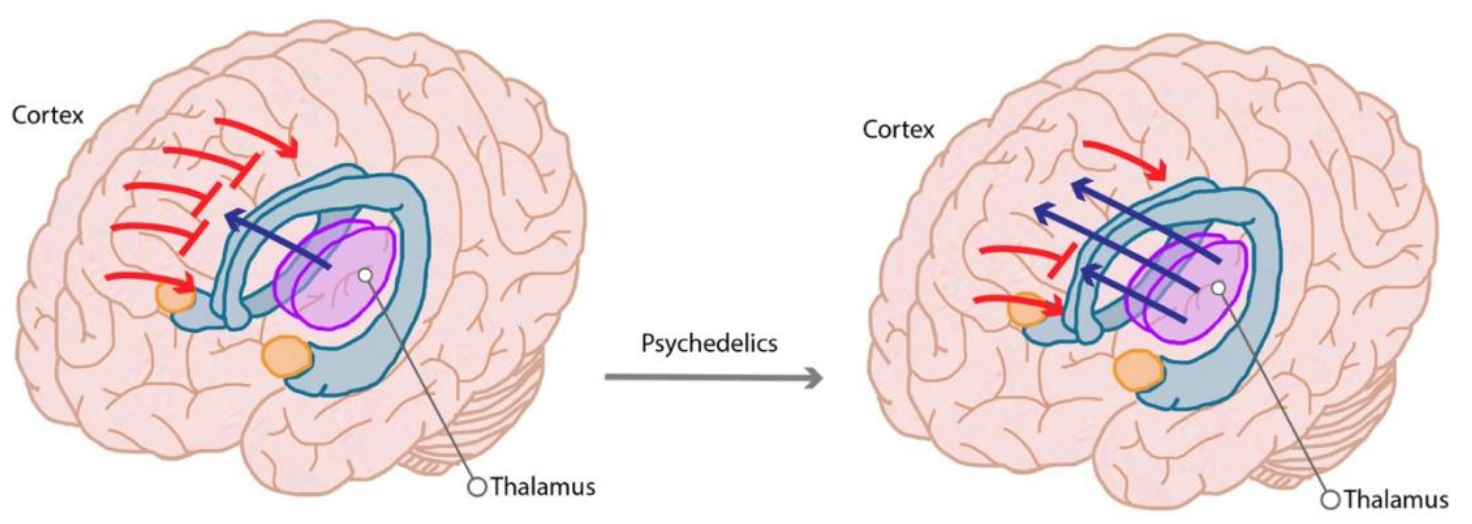

Figure 5. Opening the thalamic filter under psychedelics. Flat heads represent top down inhibition of bottom up signals, arrow heads represent uninhibited signals. Reduced top down inhibition from the cortex enables increased bottom up connectivity to the cortex. 
The MTL regions involve limbic structures that are associated with emotion and memory, which may relate to the therapeutic effects of psychedelics (R. Carhart-Harris, 2007; Ritchey, Wang, Yonelinas, \& Ranganath, 2019). Cortical midline structures such as the PCC, anterior cingulate cortex (ACC) and medial prefrontal cortex (mPFC) are connected to MTL structures including the parahippocampus (PHC), retrosplenial cortex (RSC) and amygdala (AMG) (Andrews-Hanna, Smallwood, \& Spreng, 2014; Amit Etkin, Egner, \& Kalisch, 2011; Milad \& Quirk, 2002). Like the thalamus, increased signalling from the MTL following psychedelics is thought to affect the influence of MLT on higher associative areas and subsequently conscious awareness (R. L. Carhart-Harris et al., 2014). Abnormal MTL activity can produce dreamy states and sense of depersonalisation, resembling psychedelic subjective effects (Halgren, Walter, Cherlow, \& Crandall, 1978; Lemche et al., 2016). This suggests psychedelic-induced alterations of the MTL may produce similar effects. However, the MTL's importance in memory and emotion point to its connectivity with other cerebellar and cortical regions that may play a role in acute and lasting therapeutic effects. Moreover, decoupling between cortical regions and the MTL has been suggested to play a role in ego dissolution (Alexander V. Lebedev et al., 2015). Understanding connectivity changes of the MTL under psychedelics may help discern its contribution to subjective and therapeutic effects. Partitioning of the MTL into its constituent systems, including the AMG, PHC and RSC, provides a more granular view of their role under psychedelics.

\section{Amygdala}

The AMG is involved in perception and emotional processing (Kraehenmann et al., 2015; Phelps \& LeDoux, 2005) and identification of emotionally salient stimuli. Activity of the AMG can be summarised as eliciting experiences of heightened emotion mediated by top-down connectivity. Moreover, the AMG may play a role in mediating visual salience. This emotional 
processing circuitry can be sensitive to the effects of psychedelics. The AMG receives topdown projections from midline cortical regions, like the ACC with indirect connections to the mPFC (Amit Etkin et al., 2011; Kraehenmann et al., 2016; Robinson et al., 2013). For example, top-down connectivity between the ventral ACC and AMG influences emotional regulation and reappraisal (Amit Etkin et al., 2011). AMG circuitry is thought to respond to threats to both beliefs and physical safety (Kaplan, Gimbel, \& Harris, 2016). This implicates the AMG in ego resistance and defences that may require resolution to produce ego dissolution experiences. However, the AMG's association with anxiety and fear should not overshadow its more general role in the regulation of emotions (Price, Cole, \& Goodwin, 2009) and detection of emotional salience (Adolphs, 2010; Santos, Mier, Kirsch, \& Meyer-Lindenberg, 2011). AMG activation does not determine the emotional valence of subjective experiences. For example, increased AMG activation was found after treating (treatment-resistant) depression with psilocybin (Roseman, Demetriou, Wall, Nutt, \& Carhart-Harris, 2018). This effect was attributed to acceptance and emotional reconnection reported by patients (Roseman, Demetriou, et al., 2018; Watts, Day, Krzanowski, Nutt, \& Carhart-Harris, 2017). Conversely, another psilocybin study associated positive mood change in healthy subjects with decreased AMG response measured acutely (Kraehenmann et al., 2015). This suggests AMG deactivation was associated with a reduction in negative emotion and aligns with other psilocybin investigations showing a reduction of cerebral blood flow in the AMG that correlates with a reduction of depressive symptoms (R. L. Carhart-Harris et al., 2017).

Such contradictory associations between AMG activation and behavioural outcomes are evident throughout imaging literature. The reduced structural volume of the AMG in meditators is thought to represent decreased negative affect (Gotink et al., 2018) while the same finding in borderline personality disorder is believed to indicate decreased emotional regulation (Weniger, Lange, Sachsse, \& Irle, 2009). These findings suggest the structural volume and activation of the AMG in isolation do not disambiguate the quality of psychological experience. More accurate associations between the AMG and behaviour require attention to the subject's 
experience during measurement and the time when AMG activity is assessed: the AMG appears to deactivate under acute psychedelic effects in response to negative emotional stimuli in participants in supportive conditions. Increased activation of the AMG however appears in the period following clearance of the drug.

Understanding top-down connectivity may help disambiguate the changes in AMG relating to psychological function and therapeutic outcomes. Top-down connectivity generally has an inhibitory influence on bottom-up projections. Disruption caused by psychedelics to top-down connectivity is seen clearly in changes in the visual-limbic-prefrontal network involved in detecting visual threat cues. This circuitry is thought to evince increased top-down influences in pathological conditions (Disner, Beevers, Haigh, \& Beck, 2011). Under acute effects of psilocybin, reduced top-down threat processing in healthy adults is demonstrated by decreased effective connectivity from the AMG to the primary visual cortex (Kraehenmann et al., 2016). Cortical connectivity to the AMG also mediates emotional threat processing and LSD reduces AMG responses to fearful faces in healthy adults, under acute effects (Mueller et al., 2017).

As previously mentioned, an opposite pattern of increased AMG activity is seen using similar task conditions one day after psilocybin in the (right) AMG (Roseman, Demetriou, et al., 2018) while individuals with borderline personality disorder - which is characterised by over-reactive responses to threat-show hyper AMG activation to emotional face tasks (Donegan et al., 2003). This signifies the important role of top-down connectivity when interpreting AMG activity. Follow-up research measuring psilocybin effects one day after intake suggests AMG activity increases are accompanied by decreased connectivity between the prefrontal cortex and the AMG (Mertens et al., 2020). Although this research did not determine the direction of influence, the authors propose connectivity involving the AMG may be an important mechanism underlying previously reported therapeutic outcomes of emotional reconnection (Watts et al., 2017). 
AMG and affect responses to negative faces have also been measured up to one month post psilocybin in healthy participants. This research shows sustained reduction of AMG responses to negative faces lasting up to one month. Moreover, this research indicated increased positive responses to emotionally conflicting (resistance arousing) stimuli, lasting up to one week, were associated with cortical projections to the dorsal lateral prefrontal and medial orbitofrontal cortex (F. S. Barrett, Doss, Sepeda, Pekar, \& Griffiths, 2020). Positive responses to emotionally conflicting (resistance arousing) stimuli-involving the cortex and changes to the AMG-are evidence that psychedelics can have enduring effects on neuroplasticity, lasting well beyond the half-life of psilocybin. These results also speak to the importance of emotional regulation of the AMG by the cortex in long-lasting therapeutic outcomes. Reduced acute activation of the AMG by negative stimuli, under psychedelics, may contribute to reduced ego resistance and positively felt ego dissolution.

\section{Parahippocampus and hippocampus}

The PHC is an associative hub of the MTL involved in memory retrieval, associative memory, contextual processing and detection of familiarity (Aminoff, Kveraga, \& Bar, 2013). The hippocampus $(\mathrm{HC})$ is involved in decision-making, learning and memory consolidation and can be further divided into the dentate gyrus, the hippocampus proper, and the subiculum (Anand \& Dhikav, 2012; Fogwe LA, 2021). The PHC is connected with the HC and may mediate HC connections with the associative cortex (Millière, 2017; Ward et al., 2014). This connectivity is relevant in limbic-cortical dysregulation impacting mood regulation (Zeng et al., 2012) and increased FC between the HC and prefrontal cortex (e.g., mPFC) is seen in depression (Kaiser, Andrews-Hanna, Wager, \& Pizzagalli, 2015). Decreased resting state FC between the PHC and prefrontal cortex (the PFC) was identified under psilocybin and was predictive of treatment response in a study of psilocybin for treatment-resistant depression ( $R$. L. Carhart-Harris et al., 2017). Moreover, these connectivity changes also predicted ego dissolution (R. L. Carhart-Harris et al., 2017). Dysconnectivity of the PHC is also implicated in medial temporal lobe epilepsy and deja-vu experiences (IIIman, Butler, Souchay, \& Moulin, 
2012), suggesting, under psychedelics, PHC activity in the detection of familiarity may contribute to disruption of the narrative self (discussed later). Following this notion, information theoretic analysis of fMRI brain connectivity identified that reduced connectivity (as measured by lower diversity coefficient) between the anterior PHC and cortical regions increased the likelihood of experiencing ego dissolution under psilocybin (Alexander V. Lebedev et al., 2015). Speculatively, reduced connectivity may sensitise neuronal message passing to the effects of $5 \mathrm{HT} 2 \mathrm{AR}$ activation.

Music also influences PHC connectivity with cortical regions under psychedelics and may relate to $\mathrm{PHC}$ function in associative memory and contextual processing. A study using DCM for $\mathrm{fMRI}$ data reported increased connectivity of the PHC to visual regions and attenuated topdown connectivity under LSD (Kaelen et al., 2016). This suggests that psychedelic-induced dissolution of hierarchical processing may decouple the PHC and the visual system (Kaelen et al., 2016). It also suggests free interactions lower in the cortical hierarchy-labelled anarchy (R. Carhart-Harris, Kaelen, \& Nutt, 2014).

Oscillatory changes are also seen in psychedelic states. Cortical oscillations are rhythmic electrical activity or waves that are produced by interactions among neurons. Synchronous patterns of neuronal firing are commonly associated with states of arousal and sleep (Poulet \& Crochet, 2019). EEG recordings under psilocybin show decreased PHC oscillations-also observed in coherence between the ACC and PCC (Kometer, Pokorny, Seifritz, \& Volleinweider, 2015). These findings suggest changes to cortical FC and the PHC contribute to the psychedelic experience. The PHC also contributes to spatial navigation functions of the retrosplenial cortex (RSC) (Epstein, 2008; Vann, Aggleton, \& Maguire, 2009).

\section{Retrosplenial cortex}

The RSC supports a sense of head orientation and cognitive capacities such as visualisation, autobiographical memory, future-oriented thinking, retrieval of memory, and navigation. RSC's role in navigation enables awareness of self-location, relative to spatial context, and the 
translation of spatial context to cognitive maps, which are independent of self-reference (Vann et al., 2009). The role of RSC in generating reference independent self-orientation has also been suggested to extend to representations of permanence; both of environmental features (Auger, Mullally, \& Maguire, 2012) and representations of permanence more generally (Auger \& Maguire, 2018; Kim \& Maguire, 2018). These representations may underwrite a sense of self, time and space that are affected by ego dissolution. A study of LSD, integrating fMRI, MEG and arterial spin labelling (ASL) MRI, showed decreased communication between the RSC and PHC, which correlated with subjective measures of ego dissolution and the maintenance of a sense of self (R. L. Carhart-Harris, Muthukumaraswamy, et al., 2016). Desynchronisation of delta band oscillations, measured between RSC, PHC and lateral orbitofrontal areas, were also seen under psilocybin: this desynchronisation was associated with spiritual experience and insight, which may be considered aspects of ego dissolution (Kometer et al., 2015). It was conjectured that the implicit effects on connectivity may facilitate insight-enhanced reprocessing of autobiographical memories (Kometer et al., 2015). Conversely, memory retrieval tasks under psilocybin do not support this supposition (R. L. Carhart-Harris, Leech, et al., 2012). However, it is worth noting that the memory retrieval studies included only a few subjects $(n=10)$ and did find lasting therapeutic effects of psilocybin (R. L. Carhart-Harris, Leech, et al., 2012). Despite the absence of clear evidence for the functional correlates of psychedelic effects on the RSC, its role in spatial orientation and representation of permanence suggests a role in ego dissolution.

\section{Subcortical summary}

Evidence relating hierarchical connectivity changes between subcortical limbic regions and the cortex under psychedelics is consistent across studies and may relate to ego dissolution and therapeutic effects. However, further investigations are needed to identify the specific effects on directed (effective) connectivity and their functional role in sentience and ego dissolution. The effects on thalamic connectivity appear to be an increase in bottom-up 
connectivity, which may facilitate hallucinations/hallucinosis through reduced sensory filtration. The role of the thalamus role in ego dissolution and the cognitive aspects of psychedelic experiences is uncertain and may be indirect. The connectivity of the AMG with cortical and visual regions speak to an effect of psychedelics on emotional regulation. This connectivity also appears to be rendered increasingly plastic by psychedelics. In positively felt ego dissolution, connectivity between the AMG and cortical regions may facilitate reduced threat responses (defences) and ameliorate ego resistance required for therapeutic outcomes. The effect of psychedelics on associative processes in the PHC may have a role in ego dissolution and mediate therapeutic effects through routing of neuronal signals between limbic structures and the cortex. Psychedelics may also decouple the PHC from regions lower in the hierarchy, due to reduced top-down inhibition from higher levels of the cortex. The ensuing flexibility, or anarchy, may also account for increased context-sensitivity experienced by subjects under psychedelics. A role of the RSC in the dissolution of ego permanence is conjectured. The RSC is related to sense of permanence and may mediate some of the therapeutic effects of psychedelics.

Changes in subcortical connectivity, with higher cortical areas, indicate the importance of hierarchical message passing under psychedelics. Cortical regions support high-level processes including self-related thinking, salience and attention and are responsible for topdown connectivity, which influences bottom-up projections from subcortical regions. The major cortical networks — that feature in the psychedelic imaging literature-are considered next.

\section{Connectivity within large-scale resting state networks}

The default mode network

The default mode network (DMN) is a brain network that shows increased endogenous fluctuations when an individual is awake but not engaged in a task requiring attention 
(Buckner, Andrews-Hanna, \& Schacter, 2008). It was originally identified using PET and fMRI which showed decrease in activity from baseline-in certain brain regions-during goal directed behaviour. This decreases regional activity in the absence of attentional and stimulusbound processing (Raichle et al., 2001). Advances in our understanding of the role of DMN rests on its connectivity to other parts of the association cortex (Bonnelle et al., 2012; Hagmann et al., 2008). DMN connectivity is believed to emerge over the course of human evolution and at a developmental timescale over the lifespan (Supekar et al., 2010; Van Essen \& Dierker, 2007).

The free energy principle, and associated Bayesian brain hypotheses, rests on the notion that the brain is trying to fit or invert an internal model that generates the sensory consequences of hidden or latent states in the world. On this view, the brain embodies a hierarchical generative model of how sensations are generated. In terms of evolution, hierarchical brain organisation is thought to reflect recently evolved high-level associative functions that have a predictive, supervisory and directive role over subordinate primal regions (Holmes \& Nolte, 2019). The notion of hierarchy suggests an increased complexity of representations from sensory to associative regions of the brain (Felleman \& Van Essen, 1991). Furthermore, complex sensory events involving longer sequences of representations are processed in superordinate levels of the hierarchy capable of generating ordinal sequences and narratives (Kiebel, Daunizeau, \& Friston, 2008). In predictive coding formulations of the Bayesian brain, the highest levels of the hierarchy are thought to generate top-down predictions of sensory information. The DMN is believed to be situated at the top of the cortical hierarchy and may control bottom up (prediction error) signals from lower levels of the hierarchy (e.g., the MTL) (Andrews-Hanna, Reidler, Sepulcre, Poulin, \& Buckner, 2010; Menon, 2011). The cycle of updating top-down predictions based on the flow of bottom-up prediction errors is referred to as hierarchical predictive coding (K. Friston, 2018; Ryota Kanai, Yutaka Komura, Stewart Shipp, \& Karl Friston, 2015; Rao \& Ballard, 1999). 


\section{DMN function and priors (beliefs)}

The function of the DMN centres on adaptive and internally oriented mental processes that allow humans to be aware of environmental threats such as predators, in the absence of goal directed attention (Raichle et al., 2001). The DMN's role in directing attention inward-that manifests as self-oriented thoughts—also suggests its relationship to personal identity and the construct of narrative self, which is closely related to the Freudian concept of ego (Buckner et al., 2008; R. L. Carhart-Harris \& Friston, 2010; Alexander V. Lebedev et al., 2015).

The DMN is rich in 5HT2AR (Beliveau et al., 2017). Mounting evidence suggests psychedelics decrease DMN connectivity; see Table 1, which provides a summary of connectivity changes under various psychoactive compounds (Ruban \& Kolodziej, 2018). Acute decrease in DMN connectivity is observed under both psilocybin and LSD (R. L. Carhart-Harris, Erritzoe, et al., 2012; Felix Müller et al., 2018). Decreased DMN connectivity likely relates to ego dissolution and may involve the dissolution of self-related priors (beliefs) sustained by the DMN. These priors may support a narrative sense of self that disintegrates during ego dissolution. Selfrelated priors in the DMN are also thought to control the top down inhibition of prediction errors from subordinate hierarchical levels (Dillon \& Pizzagalli, 2007) (R. L. Carhart-Harris \& Friston, 2010) (R. L. Carhart-Harris et al., 2014). If top down inhibitory mechanisms are disengaged by psychedelics, then DMN regions may become more receptive to bottom up influences (e.g., from the MTL) that enable revision and updating of beliefs (R. L. Carhart-Harris \& Friston, 2019).

Table 1. DMN (default mode network) connectivity under classic psychedelics, inspired by Ruban \& Kolodziej, 2018. Decreased DMN activity under psychedelics is a common finding across psychedelic literature. For DMN functional connectivity with other regions see (Ruban \& Kolodziej, 2018).

\begin{tabular}{|c|c|c|c|}
\hline Author & $\frac{\text { Within-DMN }}{\text { connectivity }}$ & Substance & $\underline{\text { Time of scanning }}$ \\
\hline $\begin{array}{l}\text { Carhart-Harris et al., } \\
2016\end{array}$ & Decreased & LSD & Under psychedelic \\
\hline
\end{tabular}




\begin{tabular}{|c|c|c|c|}
\hline Author & Within-DMN & $\underline{\text { Substance }}$ & Time of scanning \\
\hline & connectivity & & \\
\hline Muller et al., 2018 & Decreased & LSD & Under psychedelic \\
\hline $\begin{array}{l}\text { Palhano-Fontes et } \\
\text { al., } 2015\end{array}$ & Decreased & Ayahuasca & Under psychedelic \\
\hline $\begin{array}{l}\text { Carhart-Harris et al., } \\
2012\end{array}$ & Decreased & Psilocybin & Under psychedelic \\
\hline $\begin{array}{l}\text { Carhart-Harris et al., } \\
2017\end{array}$ & Increased* & Psilocybin & Day after psychedelic administration \\
\hline
\end{tabular}

\section{DMN and priors in mental health}

Maladaptive priors are surmised to be part of ego resistance. Disinhibition of ascending prediction errors by psychedelics may reduce ego resistance and play a permissive and foundational role in the updating of priors. Reported psychedelic therapeutic outcomes and increased communication between the subcortex and cortex is reflected in their role in the cognitive control of emotion (Ochsner \& Gross, 2005). The interaction between cognition and emotion may help disarm maladaptive ego defences. In the context of psychedelic therapy, supportive preparation and prior application of behavioural therapies may also disarm maladaptive ego defences and help guide positively felt ego dissolution and facilitate cognitive-emotional engagement (R. L. Carhart-Harris et al., 2018), emotional reconnection (Watts et al., 2017) and a sense of being attuned with one's emotions (Roseman, Demetriou, et al., 2018).

The importance of DMN connectivity in mental wellbeing is evidenced in psychopathology research. For example, connectivity variability between the mPFC and PCC is greater in depressed individuals than in healthy controls (Wise et al., 2017) and increased connectivity of the DMN is associated with ruminative thought, low mood and depression (Speth et al., 2016). Furthermore, reduced task-related mPFC suppression and increased functional connectivity of the DMN has been identified in schizophrenia (Whitfield-Gabrieli \& Ford, 2012; 
Whitfield-Gabrieli et al., 2009) and decreased anterior and increased posterior DMN connectivity is reported in addiction (Zhang \& Volkow, 2019).

These reports speak to the importance of DMN connectivity for psychological wellbeing. For example, decreases in DMN functional connectivity is related to reduced references to the past by subjects post-LSD in task-based studies, suggesting that the influence of psychedelics on DMN function may relate to rumination (Speth et al., 2016). This could mean psychedelics—similar to meditation-cultivate a more present-centred focus (Brewer et al., 2011). Moreover, this present-centred focus may represent reduced defence mechanisms. Investigation of the brain regions that form DMN and their relationship with self-related processes may help discern mechanisms of action of psychedelics that mediate ego dissolution and therapeutic outcomes.

\section{Posterior cingulate cortex}

Within the DMN, a cardinal structure associated with the self is the PCC. The PCC absorbs around $20 \%$ more metabolic energy than most other brain regions (Buckner et al., 2008; Raichle et al., 2001). Its prominent role may be reflected in its structurally fortified location and access to ample blood supply (Raichle et al., 2001). The PCC remains active: automatically and continuously alert to surroundings (Raichle et al., 2001) and there is robust evidence of PCC involvement in processes of self-reflection (B. A. Vogt \& Laureys, 2005) and a sense of self (Sampedro et al., 2017). This suggests its activity underwrites a narrative sense of self (the ego) (R. L. Carhart-Harris, Erritzoe, et al., 2012; R. L. Carhart-Harris \& Friston, 2010). The PCC is also a hub that routes and regulates neuronal message passing throughout the brain and has been shown to be abnormal in schizophrenic patients (Calhoun, 2011).

The importance of the PCC in the preservation of ego was suggested by an fMRI study of regional decreases of cerebral blood flow in the PCC (by up to $20 \%$ ) that were correlated with 
ego dissolution induced by psilocybin (R. L. Carhart-Harris, Erritzoe, et al., 2012). The relationship between the PCC and ego dissolution was further characterised by effective connectivity analysis of MEG data using DCM. DCM analysis demonstrated that psychedelics desynchronised cortical oscillatory rhythms of the PCC by decreasing alpha power (R. L. Carhart-Harris, Muthukumaraswamy, et al., 2016; Muthukumaraswamy et al., 2013). This finding suggested that the alpha $(8-13 \mathrm{~Hz})$ frequency band of the PCC may be a correlate of ego integrity (R. L. Carhart-Harris et al., 2014). This observation was reinforced by later findings, from fMRI under LSD, which also detected reduced alpha power in the PCC in association with ego dissolution (R. L. Carhart-Harris, Muthukumaraswamy, et al., 2016).

PCC functionality is differentiated by its ventral and dorsal segments. The ventral PCC shares connections with the DMN during internally focused states and is thought to orient attention towards the self, while the dorsal PCC - a node within multiple resting state networks -is involved in the dynamic coordination of attentional focus between internal and external thoughts by altering the metastability of resting state networks (Leech \& Sharp, 2014; Katrin H. Preller et al., 2019). The connectivity of the dorsal and ventral PCC with other brain regions-in conjunction with evidence of its alteration by psychedelics-suggests it may be important to track these pathways to understand the mechanisms of psychedelic effects. Evidence of connectivity between the PCC and another cardinal node of the DMN, the mPFC, has also been highlighted in accounts of ego dissolution.

\section{Connectivity to the medial prefrontal cortex}

The mPFC is implicated in executive cognitive functions, involving memory and decision making (Euston, Gruber, \& McNaughton, 2012), and contextual associations between memory and responses by relating times, places, and events to adaptive emotional and physical responses (Euston et al., 2012). The mPFC integrates bottom-up internal and external information, conveyed by connectivity to the PCC, which may play a self-reflective role in 
decision making (Brewer et al., 2011; Gusnard, Akbudak, Shulman, \& Raichle, 2001; Northoff et al., 2006). Relatedly, functional connectivity between the PCC and mPFC is identified in post-decision dissonance (Tompson, Chua, \& Kitayama, 2016). The mPFC also has a role in selecting which bottom-up signals reach the DMN, which points to its significance in emotional processing (Gusnard et al., 2001; Ochsner \& Gross, 2005). Under LSD, evidence of mPFC emotional mediation is demonstrated by reduced reactivity of right $\mathrm{mPFC}$ and left AMG functional connectivity in response to fearful faces (Mueller et al., 2017). Neuroimaging studies support the role of the mPFC in the suppression of limbic signals (e.g., in post traumatic stress disorder) (R. L. Carhart-Harris \& Friston, 2010; Davidson, Putnam, \& Larson, 2000; A. Etkin, Egner, Peraza, Kandel, \& Hirsch, 2006; Hariri, Bookheimer, \& Mazziotta, 2000; Hopper, Frewen, van der Kolk, \& Lanius, 2007; Milad \& Quirk, 2002; Phillips, Drevets, Rauch, \& Lane, 2003; Shin et al., 2004). In short, changes in the mPFC facilitation of bottom-up signals, under psychedelics, may be important in ego dissolution and therapeutic outcomes.

Mindfulness research has identified reduced mPFC and PCC connectivity in meditators following training, compared to control subjects (Brewer et al., 2011; Simon \& Engström, 2015). Similarities between psychedelic experiences and meditation practice provides a reference for psychedelic-induced connectivity changes and therapeutic outcomes, and moreover suggest a potential synergy. Smigielski et al. report a study in which experienced meditators practiced an open-awareness style of meditation under a single dose of psilocybin during a five-day mindfulness retreat (Smigielski, Scheidegger, et al., 2019). These meditators then underwent $\mathrm{fMRI}$ imaging one day after the retreat. Compared to controls, meditators exhibited reduced mPFC-PCC connectivity that correlated with ego dissolution. Moreover, lasting therapeutic outcomes were evident four months after administration. These results are significant because, despite being trained with an average $5000+$ hours, the treatment group showed lasting psychological gains beyond the benefits of extensive meditation alone (Smigielski, Scheidegger, et al., 2019). Interestingly, during the follow up fMRI, meditators in the psilocybin group were able to better modulate experiences of self-transcendence through 
their meditation associated with the mPFC-PCC decoupling (Smigielski, Scheidegger, et al., 2019) suggesting psychedelic-induced neural plasticity in these projections. This study also noted reduced connectivity between the MPFC and angular gyrus, another node of the DMN, that was associated with a loosening of self-reference (Smigielski, Scheidegger, et al., 2019). Increased self-transcendence has also been associated to cortical thinning of the PCC in regular ayahuasca users (Carlos Bouso et al., 2015). These research findings again highlight that psychedelics may facilitate lasting changes-in this case, self-transcendence through mPFC-PCC connectivity under meditation-that may also facilitate longer term psychological wellbeing.

An earlier fMRI psilocybin study found decreased functional connectivity between the PCC and mPFC. The link was interpreted as a reduced PCC influence on the mPFC and was related to ego dissolution (R. L. Carhart-Harris, Erritzoe, et al., 2012). However, this assertion was not supported by an effective connectivity analysis of the directed influence. Another study using ayahuasca failed to find significant decoupling between PCC and mPFC using fMRI. Instead, and similar to the research of mindfulness meditators (Brewer et al., 2011), the PCC and mPFC showed reduced self-connectivity (Palhano-Fontes et al., 2015). The deactivation of mPFC has been related more generally to the subjective effects of psilocybin (R. L. Carhart-Harris, Erritzoe, et al., 2012; Ruban \& Kolodziej, 2018). Decreased connectivity within or between the MPFC and PCC may indicate self-related attention, contextual judgement, emotional regulation and suppression of bottom-up signals as possible mechanisms of ego dissolution. This evidence points to the importance of the midline regions of the DMN under psychedelics. However, further specification of the roles of these connections is required before any more definitive conclusions can be drawn.

\section{Salience network}


The salience network (SN) serves important roles in sentience and conscious awareness. It detects and evaluates salient events (Menon \& Uddin, 2010), monitors the environmental features relevant to goal-directed thinking (Seeley et al., 2007) and acts as the switching mechanism, coordinating attention between internal and external stimuli (Liang et al., 2015) (Seeley et al., 2007). Moreover, the SN contributes to self-awareness (Menon, 2015). The SN is usually considered to be composed of the dorsal anterior cingulate cortex (dACC) and anterior insula (Al). Both are consistently coactivated across cognitive tasks (Swick, Ashley, \& Turken, 2011), although the dACC is more involved in response selection and conflict monitoring (Ide, Shenoy, Yu, \& Li, 2013) (Menon, 2011). The Al receives more multimodal sensory input (Averbeck \& Seo, 2008) (Brent A. Vogt \& Pandya, 1987), detects behaviourally relevant stimuli (Menon, 2015), and coordinates the dynamic interactions of networks involved in external oriented attention and internally self-oriented mental processes (Menon \& Uddin, 2010; Sridharan, Levitin, \& Menon, 2008). The SN is also associated with reasoning under uncertainty (Donoso, Collins, \& Koechlin, 2014; Alexander V. Lebedev et al., 2015; Singer, Critchley, \& Preuschoff, 2009), perception of time (Craig, 2009) and self-agency (Farrer \& Frith, 2002). Many of these associations share a similarity to ego dissolution effects under psychedelics. Psychedelic-induced alterations of self-agency, the perception of time (Shebloski \& Broadway, 2016) and reasoning under uncertainty indicate a possible role for the $\mathrm{SN}$ in the awareness of psychedelic experiences.

\section{Anterior cingulate cortex}

The ACC is a cortical midline hub region of the $S N$ involved in cognitive control and is frequently highlighted in connection with psychedelic-induced altered states. It is highly connected to hub regions of the DMN and plays a role in self-referential processing (Northoff et al., 2006). It is also situated between emotional and cognitive domains suggesting it may bridge and mediate emotional responses (Liotti et al., 2000) (Amit Etkin et al., 2011; Stevens et al., 2011). Similar to the PCC, the ACC is a large region with dorsal and ventral subdivisions. 
The dACC is connected to regions implicated in cognition, such as the (lateral) prefrontal cortex, parietal cortex, and premotor and supplementary motor areas (Asemi, Ramaseshan, Burgess, Diwadkar, \& Bressler, 2015; Heilbronner \& Hayden, 2016). dACC connectivity may be expressed in social pain. A task-based fMRI study exposed controls and participants under psilocybin to social exclusion cues. Under psilocybin, negative responses to social exclusion were reduced with decreased dACC connectivity (K. H. Preller et al., 2015). The dACC is a large cortical region with multiple functions; including performance monitoring (Ham et al., 2014). This may suggest reduced dACC activity represents abnormal self-monitoring in response to social cues under psilocybin. Changes in AMG responsiveness under psychedelics suggests that changes to top-down mechanisms may indeed mediate emotional responses (Katrin H. Preller et al., 2020) with the ACC implicated (Fusar-Poli et al., 2010) (Ghashghaei, Hilgetag, \& Barbas, 2007). Moreover, the rostral ACC (rACC) sub-area of the ACC is related to emotional expression (A. Etkin et al., 2006) and-under psilocybin-has been related to subjective scores on emotional subcategories of the 5D-ASC (more on this below) (Lewis, Preller, Braden, Riecken, \& Vollenweider, 2020). Magnetic resonance spectroscopy (MRS), after ingestion of ayahuasca, showed increased connectivity between the ACC and MTL regions associated with enhanced self-compassion (Sampedro et al., 2017). Increased connectivity between the ACC and PCC was also detected. This study suggested the ACC mediated the interaction between cognition and emotion that contributes to psychedelic afterglow effects and the enhanced mindfulness capacities measured in study participants. Mindfulness capacities cultivate a more present-centred default mode (Brewer et al., 2011), develop increased awareness of mental contents (Dahl, Lutz, \& Davidson, 2015; Schooler et al., 2011), and reduce maladaptive DMN activity associated with various forms of psychopathology (Ramirez Barrantes et al., 2019; Sampedro et al., 2017; Soler et al., 2014). Mindfulness functions may also help reduce ego resistance and their increase following psychedelics again suggests evidence of neuroplasticity. 
Despite its large size and differing functions, ACC subdivisions are not always reported in psychedelic literature (Stevens et al., 2011). This may contribute to conflicting findings. For example, past PET imaging evidence describes increased ACC and MTL activity under psilocybin (F. X. Vollenweider et al., 1997). However, contrasting evidence of reduced ACC neural oscillations in a network involving the PHC, PCC and ACC, measured by EEG, have also been reported under psilocybin (Kometer et al., 2015) while further evidence suggests increased synchronisation and entropy between the ACC and hippocampus, under psilocybin (Tagliazucchi et al., 2014). The lower spatial resolution of PET and EEG may explain these contradictory findings. Spatial specificity of ACC changes under psychedelics may help identify associations between its connectivity, function and phenomenology under psychedelics. For example, the ventral stream of ACC connects to affective brain regions including the AMG (Andrews-Hanna et al., 2014; Amit Etkin et al., 2011) that could be investigated in relation to therapeutic outcomes under psychedelics.

The structural size of the ACC has also been related to psychedelics. The subjective effects of a standardised psychedelic dose can vary widely across participants and this variance may be related to the structural volume of the rACC; previously noted in subjective scores on emotional subcategories of the 5D-ASC. rACC volume correlated with inter-individual subjective effects, suggesting its structural variation across volunteers may predict feelings of unity, bliss, spiritual experience, and insightfulness (Lewis et al., 2020). Structural increases of the ACC were noted in long term ayahuasca users and may be related to long term psychedelic use that is thought to preserve psychedelic induced neuropsychological changes (Carlos Bouso et al., 2015). This sample also showed reduced cortical thickness in the PCC (Carlos Bouso et al., 2015). Notably, these long-term users of ayahuasca displayed high levels of mindfulness, which may suggest a relationship between structural change of the SN and DMN following psychedelics that is related to the increased levels of mindfulness. These structural differences may have existed prior to exposure to ayahuasca and disposed 
participants towards mindfulness and psychedelic use. This highlights the potential bias in recruitment samples in psychedelic studies.

The ACC and PCC structural differences do however align with evidence of the reliance of mindfulness capacity on $\mathrm{SN}$ control over the DMN. While the DMN is responsible for mind wandering, awareness of mind wandering can be regulated by the SN. This is reflected in functional connectivity increases during awareness of mind wandering in meditation examined in fMRI (Hasenkamp, Wilson-Mendenhall, Duncan, \& Barsalou, 2012). SN hierarchical dominance over the DMN has been validated using spectral DCM analysis (K. J. Friston et al., 2014; Zhou et al., 2018) and its control over the DMN is cited as a crucial component in cognitive health and performance (Putcha, Ross, Cronin-Golomb, Janes, \& Stern, 2016). Its breakdown results in dysregulated DMN function (Bonnelle et al., 2012). SN role in performance monitoring and emotional mediation may account for the influence of psychedelics on self-awareness and affective regulation, and encourage a more mindful or present-centred focus.

\section{Frontoparietal control network}

The frontoparietal control network (FPCN) is an attention control network also known as the central executive network (CEN) or the frontoparietal central executive network (FPCEN) (Menon, 2011). It encompasses the dorsolateral and anterior prefrontal cortices, inferior parietal lobes, anterior insulae and ACC (Rens, Bode, Burianová, \& Cunnington, 2017; Vincent, Kahn, Snyder, Raichle, \& Buckner, 2008). Notably the latter two regions (anterior insulae and ACC) overlap with the SN. The lateral prefrontal cortex and inferior parietal lobule are associated with cognitive control and decision-making (Lord et al., 2018). The FPCN is also associated with access to conscious information (S. Dehaene \& Naccache, 2001) and is inhibited in states of deep sleep (Boly et al., 2008; Tagliazucchi et al., 2016). The functional 
role of the FPCN can be summarised as evaluations over time, involving uncertainty or abstraction in voluntary choices (Andrews-Hanna et al., 2014; Jin Fan, 2014; J. Fan et al., 2014; Rens et al., 2017) and involve the dorsal prefrontal and posterior parietal cortices as key regions tasked with negotiating acute uncertainty (Huettel, Song, \& McCarthy, 2005). Abnormalities in this network, following brain injury, have been associated with impaired selfawareness (Ham et al., 2014) which may indicate its relevance to ego dissolution.

Decreases of connectivity within FPCN are detected under both LSD and psilocybin (Barnett, Muthukumaraswamy, Carhart-Harris, \& Seth, 2019; Lord et al., 2018, 2019). However, the decrease of within-network connectivity is accompanied by increases in between network connectivity (i.e., deviation of functional connections from network pathways). This pattern of reduced-within and increased-between connectivity-coined as disintegration and desegregation (R. L. Carhart-Harris, Muthukumaraswamy, et al., 2016)-is documented across resting state networks under psychedelics (Varley et al., 2019) (Barnett et al., 2019) and alters the functional integration of brain regions (Petri et al., 2014), see Figure 6. FPCN desegregated connectivity is highlighted by several studies (Lord et al., 2018, 2019; Tagliazucchi et al., 2016) and speaks to the importance of connectivity in FPCN in psychedelic experiences.

'Connectome harmonics' research has revealed that patterns of psychedelic brain dynamics preserve new complex forms of coherence and stability (Atasoy et al., 2017; Lord et al., 2019; Varley et al., 2019). Increased power and energy of connectome harmonic brain states, observed under psilocybin, display higher spatial and temporal variability yet maintain novel forms of stability and have been related to an increased repertoire of brain states (Atasoy et al., 2017). Links between these changes and their relationship to disintegrated and desegregated connectivity across resting state networks are not fully understood however theories of their role upon the FPCN can be surmised. For example, evidence of FPCN desegregated connectivity (Lord et al., 2018, 2019; Tagliazucchi et al., 2016) may influence 
consciousness by enabling access to connectivity usually restricted from the FPCN. Desegregation may also be mechanistic to increased repertoires of brain states made available under psychedelics (Atasoy et al., 2017; Tagliazucchi et al., 2014). Both observations might also relate to the phenomenological richness of psychedelic experience and therapeutic outcomes thought to depend on increased access to (precise) bottom-up prediction errors necessary for the revision of priors. The coinciding disintegration of the FPCN (Barnett et al., 2019; Lord et al., 2018, 2019) and relaxation of priors (R. L. Carhart-Harris \& Friston, 2019) would also be mechanistic in this process, however may contribute to cognitive aspects of ego dissolution. Untangling the mechanistic relationship between FPCN changes under psychedelics and their role in ego dissolution needs more research; however, existing evidence points to the potential importance of its role in cognitive aspects of the psychedelic experience.
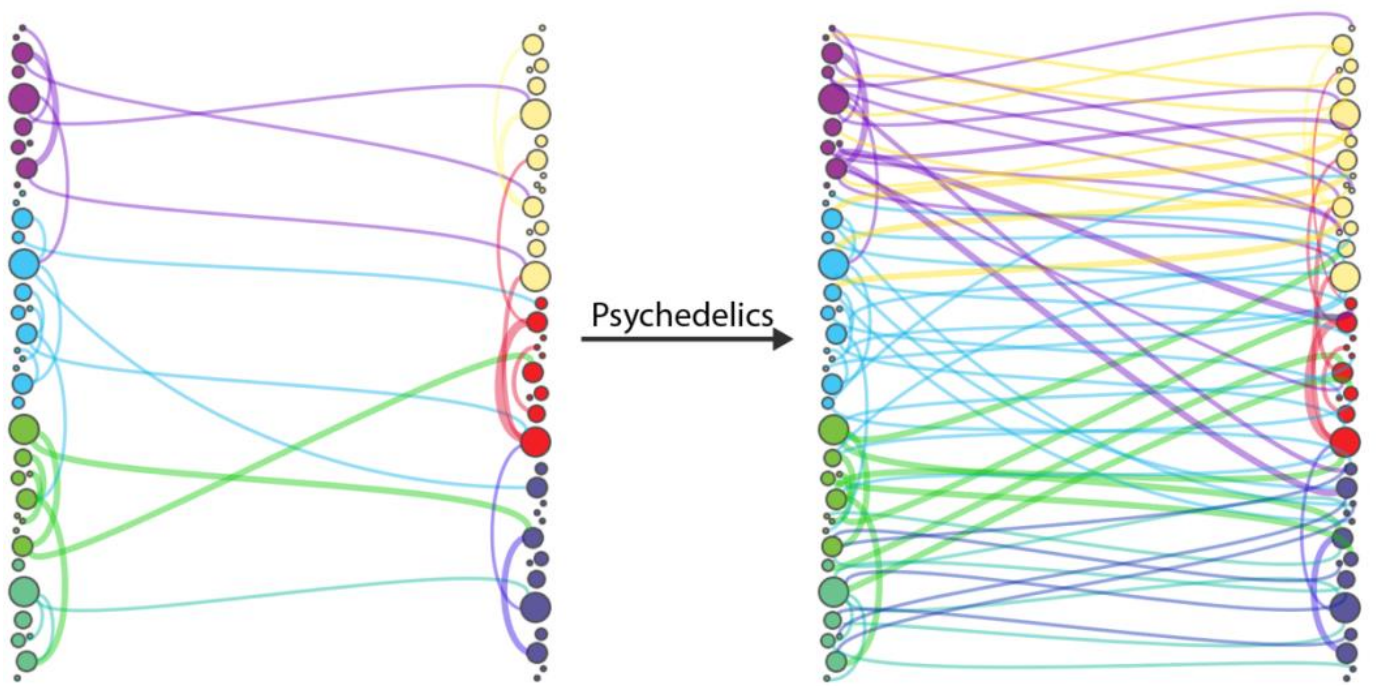

Figure 6. Illustration of desegregated connectivity under psilocybin, inspired by Petri et. al., 2014. Panel (a) illustrates integration between communities - organised by colour - observed in healthy adults. Panel b) illustrates greater integration and reduced constraint of connections between communities observed under psilocybin. For original schematic and methods see Petri et. al., 2014 (Petri et al., 2014).

Correlating cortical oscillatory changes with psychedelic experiences and therapeutic introspection may also help elucidate the role of the FPCN under psychedelics. Under 
psilocybin, an MEG study reported decreased (bilateral) beta-band activity in the FPCN (Muthukumaraswamy et al., 2013). Beta-band connectivity is suggested to encode long-term priors of behaviour and environmental stimuli (Betti, Della Penna, de Pasquale, \& Corbetta, 2020). Decreased oscillatory power of FPCN may be a measure related to change and revision of the association between behaviour and stimuli. Theta-band connectivity is proposed to support internally directed attention between the DMN and FPCN (Kam et al., 2019) and thus important in psychedelic research of brain oscillations. However, FPCN connectivity changes are not always reported under psychedelics (Felix Müller et al., 2018) and the source of inconsistencies—which are discussed later in detail—remain uncertain, although are not entirely unusual in imaging research. Added caution is further suggested by the variable functional topography of the FPCN between individuals (Marek \& Dosenbach, 2018). The importance of psychedelic effects upon the self can be explored through largescale integrated dynamics.

\section{Connectivity between large-scale resting-state networks}

The self in networks

The $\mathrm{SN}$ is related to the minimal aspects of conscious self-awareness (Limanowski \& Blankenburg, 2013) also known as the embodied aspect of self (Alexander V. Lebedev et al., 2015; Seth, 2013). The minimal or embodied aspect of self-awareness requires bottom-up processing to define physical boundaries, proprioception, interoception and the experience of oneself being rooted in (sensory) motor processes (Blanke \& Metzinger, 2009; Legrand \& Ruby, 2009). This aspect of self may be important for ego dissolution, indicated by decreased within-SN connectivity associated with ego dissolution in an fMRI psilocybin study (Alexander V. Lebedev et al., 2015). The DMN in contrast, is related to the narrative sense of self commensurate with personal identity, described in Bayesian terms as the brain predicting its own contribution to sensory input by top down inferences about the causes (i.e., latent or 
hidden states of the world) of sensory information (Clark, 2013; K. Friston, 2010; Metzinger, 2003).

Aberrant DMN-SN connectivity is important in clinical and theoretical models of various mood disorders including depression, anxiety and schizophrenia (Menon, 2011). Mood disorders associated with this connectivity suggest its importance in therapeutic outcomes and change to this connectivity under LSD is associated with positive mood and arousal (Atasoy et al., 2017). However, DMN-SN functional connectivity is more commonly linked to ego dissolution and shows reduced anticorrelation under psilocybin (R. L. Carhart-Harris, Leech, et al., 2012) and decreased segregation under LSD (R. L. Carhart-Harris, Muthukumaraswamy, et al., 2016). The relationship of DMN-SN connectivity to respective aspects of self suggests psychedelic influence on these networks may contribute to ego dissolution. Furthermore, evidence of their relationship to psychopathology speaks to the overlap between networks involved in the self and mental wellbeing. Further analysis of this connectivity and psychological changes under psychedelics may help determine the contribution of this network to ego dissolution and normal waking consciousness. These possibilities invite further investigation of the well-established anticorrelation between these networks.

\section{Coordinated balance between anticorrelated networks}

An important feature of intrinsic brain networks is their coordinated and balanced activity. Patterns of correlations and anticorrelations between networks constitute an aspect of functional integration supporting consciousness (Bak, Tang, \& Wiesenfeld, 1987; Demertzi et al., 2019). For example, the DMN focuses attention inward, in a task-free exploratory manner that—upon stimulation from the environment—rapidly deactivates in deference to externallyfocused networks like the DAN. The DAN is composed of the frontal eye fields and inferior parietal sulcus and is responsible for orientation to behaviourally salient cues (Ptak \& Schnider, 2010). Altered DAN connectivity has been reported in the psychedelic literature and 
has been related to an increased repertoire of brain states under psilocybin (Tagliazucchi et al., 2014).

The SN mediates the anticorrelation of the DMN and DAN (Fox et al., 2005) through dynamic mechanisms responsible for the coordination of attention between bottom-up perceptual features of the environment and top-down goals (Toga, 2015). The SN is hierarchically above the DAN and DMN, suggesting it may be a control network capable of selecting between these anticorrelated networks (Zhou et al., 2018). SN meditated switches enable attentional resources to transition efficiently from internal focus of DMN to an external focus (i.e., sensory attention) of DAN. Psilocybin reduces the anticorrelation between DMN-DAN connectivity deployment (R. L. Carhart-Harris, Leech, et al., 2012). Reduced anticorrelation indicates attentional resources may not be divided between external and internal realms of attention in the usual manner. Descriptions of ego dissolution that describe the dissolution of boundaries between the objective and subjective world signify the importance of investigations of the DMN-DAN anticorrelation. Like SN-DMN connectivity, competition between the DMN and DAN is correlated with disorders such as schizophrenia (Whitfield-Gabrieli et al., 2009), highlighting the potential importance of DMN-DAN anticorrelation in mental health and perceptual synthesis. A certain form of meditation, known as nondual awareness, tries to reduce the separation between external and internal reality, that intriguingly reduces the anticorrelation between brain areas that respond to intrinsic and extrinsic stimuli (Josipovic, Dinstein, Weber, \& Heeger, 2012). This finding reinforces the suggestion that DMN-DAN anticorrelation may be crucial to the loss of boundaries between the subjective and the objective world (Grof, 1980) that characterise ego dissolution.

Alterations to anti-correlated brain networks may also be involved in therapeutic outcomes following psychedelics; however, evidence is scarce. Whether therapeutic outcomes represent a direct result of altered anticorrelation or changes to connectivity between other systems such as the MTL under acute effects is unclear. It may be possible that the altered 
anticorrelations contribute to ego dissolution and promote neuroplasticity, leading to psychological changes. Furthermore, the role of these networks in higher order processing and attentional orientation could indicate access to information that is usually supressed and may contribute to therapeutic processes that reorganise patterns of thought. Evidence of DMN-DAN connectivity changes associated with enhanced mindfulness capacities may represent a link to reduced reactivity, judgemental thinking and enhanced self-kindness (Sampedro et al., 2017). Moreover anticorrelations under control of the SN also involve the FPCN (Zhou et al., 2018), which is known to be anti-correlated with the DMN (Andrews-Hanna et al., 2014; Bolton et al., 2020; Menon, 2018) (Chand, Wu, Hajjar, \& Qiu, 2017; Menon, 2011).

Control of the FPCN is managed by the SN recruitment of the anterior insula (AI), previously noted to be involved in the interactions between internal and external mental processes (Menon \& Uddin, 2010; Sridharan et al., 2008) and detect behaviourally relevant stimuli (Menon, 2015). Specifically, activation of the right Al engages the FPCN resulting in coactivation of the SN and FPCN (Greicius \& Menon, 2004; Menon, 2015; Raichle et al., 2001). While normally anti-correlated, correlated activation of the FPCN and DMN has been documented to correlate with symptoms of psychosis that relate to a disturbed sense of self and uncertainty of what is and is not real (Leptourgos et al., 2020). Changes to the functional integration of the FPCN, DMN and SN are associated with meditation practice (Doll, Hölzel, Boucard, Wohlschläger, \& Sorg, 2015) and the control of attention (Corbetta \& Shulman, 2002). These observations speak to the putative role of anticorrelations in ego dissolution that involve networks which orient attention and contribute to self and perspectival belief updating.

Subcortical brain regions that evince anticorrelation under psychedelics have also been investigated. For example, the claustrum is a subcortical region that has strong connectivity with the prefrontal cortex (S. P. Brown et al., 2017). It has been shown to activate with demanding cognitive tasks and the engagement of FPCN (Krimmel et al., 2019). A recent study showed that under psilocybin connectivity between the right claustrum and the DMN 
decreased while connectivity to the FPCN increased (Frederick S. Barrett, Krimmel, Griffiths, Seminowicz, \& Mathur, 2020). Claustrum connectivity changes may contribute to functional changes in cortical networks that support attention and self-consciousness. Further investigations comparing anticorrelation of brain networks in psychosis, meditation and classic psychedelics may help determine the functional importance of brain anticorrelations in ego dissolution, therapeutic outcomes and the consciousness of self.

\section{Cautions and methodological considerations}

\section{Conflicts within psychedelics findings}

The association between subjective effects and brain connectivity is often inconsistent among psychedelic studies. A brief overview of these discrepancies characterises the present state of understanding in psychedelic neuroscience. Ego dissolution is often associated with DMN hub regions under psychedelics. This is demonstrated by mPFC-PCC decoupling in fMRI (Smigielski, Scheidegger, et al., 2019) and decreased PCC alpha power in MEG, under psilocybin (Muthukumaraswamy et al., 2013). More generally, subjective effects under psilocybin are associated with decreases across the ACC/mPFC (R. L. Carhart-Harris, Erritzoe, et al., 2012). However, brain-wide connectivity changes are also associated with ego dissolution and extend beyond the DMN, under LSD (Tagliazucchi et al., 2016) and have been linked to connectivity between the anterior PHC and cortical communities including visual, somatosensory, salience, prefrontal and control areas under psilocybin (Alexander V. Lebedev et al., 2015). Furthermore, not all psychedelic investigations find changes to the DMN. For example, the expected changes in complexity of brain signals-measured with the fractal dimension-from DMN nodes in fMRI, under LSD and psilocybin, were not found (Varley et al., 2019). 
An LSD study employing $\mathrm{fMRI}, \mathrm{MEG}$ and arterial spin labelling (ASL) identified decreased RSC and PHC connectivity that correlated with ego dissolution and noted that activity of resting state networks such as the DMN was not correlated with ego dissolution (R. L. CarhartHarris, Muthukumaraswamy, et al., 2016). These findings challenge the reliability of DMN changes associated with ego dissolution. An EEG study also found cortical oscillations in the RSC, PHC and lateral orbitofrontal areas: however, in this study these changes were associated with spiritual experience and insightfulness and not with ego dissolution (Kometer et al., 2015). Other resting state networks suspected to have a role in psychedelic effects such as the FPCN are also sometimes reported as absent: in this case in fMRI scans under LSD (Felix Müller et al., 2018). Furthermore, effective connectivity involving the PCC was found to correlate with subjective effects of psilocybin in a DCM analysis of MEG (Muthukumaraswamy et al., 2013) yet evidence of its alteration in an fMRI study of LSD was absent (Felix Müller et al., 2018). See Table 2 for summary of connectivity results associated with ego dissolution (Millière, Carhart-Harris, Roseman, Trautwein, \& Berkovich-Ohana, 2018).

Table 2. Neural correlates of ego dissolution. Adapted from Milliere, 2018.

\begin{tabular}{|c|c|c|}
\hline Author & Substance & Correlates of ego dissolution \\
\hline Carhart-Harris et al., 2013 & Psilocybin & $\uparrow$ DMN-TPN functional connectivity \\
\hline $\begin{array}{l}\text { Muthukumaraswamy et al., } \\
2013\end{array}$ & Psilocybin & $\downarrow$ alpha power in PCC \\
\hline Lebedev et al., 2015 & Psilocybin & $\begin{array}{l}\downarrow \text { function in aPHC } \\
\downarrow \text { MTL-neocortex coupling } \\
\downarrow \text { integrity of salience network } \\
\downarrow \text { interhemispheric connectivity }\end{array}$ \\
\hline Tagliazucchi et al., 2016 & LSD & $\begin{array}{l}\uparrow \text { connectivity density in bilateral TPJ/angular gyrus } \\
\text { and insular cortex }\end{array}$ \\
\hline Carhart-Harris et al., 2016 & LSD & $\begin{array}{l}\downarrow \text { integrity of DMN } \\
\downarrow \text { PHC-RSC connectivity } \\
\downarrow \text { alpha and delta power in PCC }\end{array}$ \\
\hline Lebedev et al., 2016 & LSD & $\uparrow$ entropic brain activity \\
\hline Schartner et al., 2017 & Psilocybin & $\begin{array}{l}\uparrow \text { spontaneous MEG signal diversity (measured with } \\
\text { Lempel-Ziv complexity) }\end{array}$ \\
\hline Atasoy et al., 2017 & LSD & $\begin{array}{l}\downarrow \text { mean energy and energy fluctuations of low } \\
\text { frequency connectome harmonics }\end{array}$ \\
\hline
\end{tabular}




\section{Conflicts between psychedelic findings and non-psychedelic findings}

Occasionally psychedelic induced changes in connectivity—cited in association with subjective effects-are seen in non-psychedelic studies without subjective effects. For example, decreased activation within the DMN is cited in association to ego dissolution (R. L. Carhart-Harris, Muthukumaraswamy, et al., 2016). However, this also resembles the patterns of activity following administration of sertraline, a non-psychoactive drug (Klaassens et al., 2015; Felix Müller et al., 2018). Within the DMN, the deactivation of the PCC and mPFC has been suggested to underlie ego dissolution (R. L. Carhart-Harris, Erritzoe, et al., 2012). Although, this deactivation is also identified in response to antidepressants, with no noticeable psychoactive effects (Goldstein-Piekarski et al., 2018) and a similar activation pattern is also elicited by anxiety (Zhao et al., 2007). If DMN connectivity changes underlie alterations to perception reported under psychedelics, then we might expect similar connectivity changes to produce psychedelic-like subjective effects. Psychedelic experimental designs are at risk of inducing anxiety, especially when rapid onset of a psychedelic through intravenous injection is employed. It is therefore important to note known confounds when trying to identify neural mechanisms.

\section{Signal from the noise}

The results of neuroimaging studies suggest connectivity associations with ego dissolutionand other subjective effects of psychedelics-that have yet to be clearly determined. Examining and integrating cellular, regional, network and whole brain connectivity changes may best account for observed phenomenological changes in consciousness (Busch, Dubois, \& VanRullen, 2009; Stanislas Dehaene \& Changeux, 2011; Fahrenfort et al., 2012; Hipp, Engel, \& Siegel, 2011; Melloni et al., 2007; Palva, Palva, \& Kaila, 2005; Varela, Lachaux, 
Rodriguez, \& Martinerie, 2001). Psychedelic subjective effects will likely involve widespread changes to hierarchical connectivity subtending associative and sensory processing across the cortex and subcortex. Holistic accounts of brain changes under psychedelics - that identify reliable correlations between connectivity and subjective experience-may be followed by more thorough and advanced examinations, such as those using effective connectivity analyses. However, the accurate modelling of psychedelic brain connectivity is encumbered by challenges.

The anatomical coordinates of regions—such as the PCC—may be specified using various methods for subsequent region of interest $(\mathrm{ROI})$ based analysis. For example, independent component analysis (ICA) can be used to detect the peak activity of cardinal regions and their corresponding coordinates, or these coordinates may be borrowed from previous literature. Recent alternate methods have also been proposed, involving probabilistic mapping to improve consensus and inferences, see Dworetsky et al., 2021 (Dworetsky et al., 2021). These strategies may produce different connectivity results, when referring to the same regions. Variability due to individual differences in brain anatomy may further complicate accurate determination of anatomical coordinates. Commonly referred to networks may also be composed of different regions. Moreover, the application of global signal regression has been considered a cause of anti-correlated brain connectivity and debate over its application questions interpretations of anticorrelations (Murphy, Birn, Handwerker, Jones, \& Bandettini, 2009). Concerns also exist with the interpretation of the BOLD signal, and whether the BOLD signal is sensitive to changes in conscious experience (Laumann et al., 2017).

These concerns aside, understanding the mechanistic unfolding of processes after psychedelics can be advanced by imaging and analytic approaches. This requires standardisation of image processing pipelines including: the methods of drug administration, timing of imaging, environmental setting, sample size, balance of participant group averaged neuroanatomy and data pre-processing pipelines to clean (physiological) signals. Variation in 
pre-processing pipelines in particular can be a significant barrier to the accurate comparison of findings across neuroimaging studies. For comparison of motion correction pipelines see Parkes, Fulcher, Yücel, \& Fornito, 2018 (Parkes, Fulcher, Yücel, \& Fornito, 2018), for global signal regression see Almgren, Van de Steen, Razi, Friston, \& Marinazzo, 2020 and Aquino, Fulcher, Parkes, Sabaroedin, \& Fornito, 2020 (Almgren, Van de Steen, Razi, Friston, \& Marinazzo, 2020; Aquino, Fulcher, Parkes, Sabaroedin, \& Fornito, 2020) and for motion correction in diffusion imaging pipelines see Oldham et al., 2020 (Oldham et al., 2020). The optimisation and standardisation of these pipelines-in conjunction with replication and comparison between psychedelics-may be essential. Fortunately, growing interest in psychedelic therapeutic potential may motivate standardisation and replication.

\section{Unifying neuroimaging evidence of psychedelic mechanisms}

Closing gaps in the understanding of the neural mechanisms of subjective effects of psychedelics and formulating a unifying theory of $5 \mathrm{HT} 2 \mathrm{R}$ activity across the brain remains a goal of psychedelic research. Psychedelics have been suggested to drive the brain away from order towards a state of disorder in the entropic brain theory (R. L. Carhart-Harris et al., 2014). The flexibility of conscious states, spatial and temporal changes in connectivity and increased sensitivity to intrinsic and extrinsic perturbations under psychedelics supports this theory (R. L. Carhart-Harris et al., 2014) (R. L. Carhart-Harris, 2018a) (Lord et al., 2018, 2019). Observed criticality characteristics have used to understand the stable and coherent organisation of brain dynamics under psychedelics (Atasoy et al., 2017). Criticality is a widespread phenomenon in multi-scale and complex systems such as the brain. Criticality arises between states of order and disorder. It is marked by scale-free fluctuations that stretch from the finest to the coarsest scale and which can spontaneously jump between diverse spatiotemporal patterns (Cocchi, Gollo, Zalesky, \& Breakspear, 2017). However criticality has also been 
challenged as an unnecessarily complex explanation of drug induced states (Muthukumaraswamy \& Liley, 2018).

An empirical approach to measuring psychedelic mechanisms across the brain comes from a recent imaging study explaining broad FC patterns leading to subjective effects. This study measured time-dependent changes of psilocybin over the course of onset to peak effects at three timepoints (20, 40 and 70 minutes post oral administration) (Katrin H. Preller et al., 2020). Resting state FC was quantified using a data-driven global brain connectivity method, which suggested that psilocybin reduces the connectivity of associative regions and increases connectivity in sensory regions across subcortical and cortical areas (Katrin H. Preller et al., 2020; Franz X. Vollenweider \& Preller, 2020). The authors suggest that the disintegration of associative activity and synchronisation of sensory activity may explain subjective sensory and self-related experiences under psychedelics. Both of these theoretical and empirical accounts offer useful summaries of psychedelic subjective effects that complement an evaluation of psychedelics offered by the framework of hierarchical predictive coding.

\section{Hierarchical predictive coding}

Hierarchical predictive coding is a mechanistic account of how the brain processes information (K. Friston, 2008). It is supported by empirical evidence (Mumford, 1992; Rao \& Ballard, 1999) and informed by our understanding of synaptic communication (Adams, Stephan, Brown, Frith, \& Friston, 2013; Karl J. Friston, 2020; Hobson, Gott, \& Friston, 2021). Noting the neuromodulatory role of serotonergic neurotransmission (Picard \& Friston, 2014) and the influence of psychedelics upon these receptors, it provides a unifying account of psychedelic subjective and therapeutic effects. The premise of hierarchical predictive coding is that the brain is a constructive organ explaining (i.e., inferring) sensory impressions or the causes of input on sensory epithelia. Sensory signals from the outer world are transduced into electrical signals which ascend the brain's hierarchical architecture — in the form of prediction errorsto update (Bayesian) beliefs about hidden states in the world. These (sub personal) beliefs 
form the basis of perception and can be regarded as the brain making predictions about the causes of its sensations. As new impressions from the sensory epithelia ascend the hierarchy, the accuracy of prediction is tested against the new sensory evidence in a cyclical process described variously as predictive coding, Bayesian filtering or Bayesian belief updating. In short, bottom-up sensory evidence carries information (i.e., prediction errors) which update expectations about states of affairs (i.e., posterior perceptions based on prior beliefs). These expectations then provide revised predictions that descend through the hierarchy to resolve prediction errors at lower levels.

The computational formulation of hierarchical predictive coding goes on to consider neuromodulation in the construction of predictions, suggesting gain control (i.e., sensitivity) is a key player in selecting the most reliable or precise prediction errors for belief updating at higher hierarchical levels. Synaptic gain is thought to encode precision and synaptic gain control reflects predictions of precision that implement this (attentional) selection. Computationally, precision is the predictability of some random variable and may be interpreted as a level of confidence placed in a prediction or prediction error. At high levels of the hierarchy that may entail (declarative or experienced) conscious processing, precision refers to confidence in an inference or belief. The neuromodulation or sensitisation of neural populations is thought to determine the attenuation or augmentation of precision (i.e., confidence) in a source of information. Precision control therefore provides a synaptic mechanism describing the selection and attenuation of sensory channels and is proposed as a neurological explanation for many psychopathologies (H. Brown, Adams, Parees, Edwards, \& Friston, 2013). For example, in psychosis abnormal neuromodulation or sensitisation of neural populations may confound selection and attenuation of sensory channels, resulting in false inferences (Adams et al., 2013) (Ryota Kanai et al., 2015).

The role of psychedelics in neuromodulation derives from binding to the serotonergic $5 \mathrm{HT} 2 \mathrm{~A}$ receptors. Serotonergic receptors serve a crucial role in precision control underlying sentience 
and attention that is necessary to infer both the cause of sensory impressions and the consequences of self-initiated actions (Picard \& Friston, 2014). Imaging evidence and brain connectivity findings are consistent with the preferential stimulation of 5-HT2AR on deep pyramidal cells, which are densely distributed in visual and associative areas of the cortical hierarchy (R. L. Carhart-Harris, Erritzoe, et al., 2012) (R. L. Carhart-Harris, Muthukumaraswamy, et al., 2016) (Katrin H. Preller et al., 2018) (Roseman et al., 2016) (Katrin H. Preller et al., 2020) (Franz X. Vollenweider \& Preller, 2020) (R. L. Carhart-Harris \& Friston, 2019) (Saulin, Savli, \& Lanzenberger, 2012). Under predictive coding, these cells are thought to be the cells of origin of descending predictions to subcortical systems or lower hierarchical levels.

Consistent connectivity findings across visual and associative areas have been helpful in accounting for psychedelic hallucinogenic effects and ego dissolution, respectively. And can be framed within the hierarchical predictive coding framework. For example it has been suggested that 5 HT2AR serves a role in the active coping with stress and uncertainty $(R . L$. Carhart-Harris \& Nutt, 2017). Furthermore, links between pharmacology and psychology can be considered in terms of coordinated message passing of belief updating cortical hierarchies. For example, increased entropy and changes to synaptic gain control at the top of the hierarchy may influence the FPCN that is tasked with negotiating uncertainty and subsequent influence on cognition and priors. In short, hierarchical predictive coding provides a biological starting point to understand the effect of pharmacological interventions at the synaptic level, in terms of sentience and planning.

The leading synthesis of hierarchical predictive coding and psychedelic effects to date is the REBUS (RElaxation of Beliefs Under pSychedelics) model. REBUS describes the relaxation of precision at high levels of the hierarchy, leading to a greater influence of ascending prediction errors. In other words, a rebalancing of prior and sensory precision that enables new prior hypotheses and narratives to be engaged. In virtue of these expectations being at 
the apex of a hierarchical generative model, they are necessarily concerned with agency and active sentience of a multimodal sort and, on one reading, entail an implicit or explicit notion of self as agent.

Physiologically, the relaxation of high-level precision corresponds to a reduction in the synaptic gain or efficacy of particular regions in the cortical hierarchy. These changes are relatively consistent with neuroimaging findings, which show decreased within-network connectivity and altered oscillatory rhythms. The strength of the REBUS model is its ability to explain both the mechanisms of psychedelic subjective and therapeutic effects: see Figure 7. Understanding that synaptic efficacy depends upon neuromodulation and that neuromodulation (of synaptic gain) changes the inhibition-excitation balance of synaptic integration suggests how changes in serotonergic agonist activity can influence internalising disorders - disorders that are characterised by rigid ways of thinking and unduly precise prior beliefs. This is particularly relevant to psychiatry by suggesting psychedelics as a neuromodulator that affects the selection of ascending information-and thereby may be capable of subverting overly precise beliefs (i.e., ego resistance).

Similar adaptations of the REBUS model are described in neural annealing as reduced energy sinks (M. E. Johnson, 2019) and are surmised to enable new directions for thought patterns under psychedelics - without a complete breakdown of cognition (Atasoy et al., 2017) (Petri et al., 2014)). On this view, precision plays the role of an inverse temperature, where decreasing the precision of prior beliefs (i.e., increasing their temperature) makes it easier for higher level beliefs to jump from one to another. In other words, the energy landscape is flattened. A useful analogy that captures the flattened landscape of priors under ego dissolution-and subsequent promotion of flexibility and plasticity-is that of a skier descending a hill after a fresh snow fall: Tracks (i.e., ruts), which were preferentially travelled previously (likened to prior belief updating) are levelled by the snowfall (i.e., the grooming action of psychedelics on high level precision) resulting in a flattened landscape that enables 
travel in new directions without prior resistance. In short, psychedelics may simply flatten the 'rut' and stop belief updating getting 'stuck in a rut'.

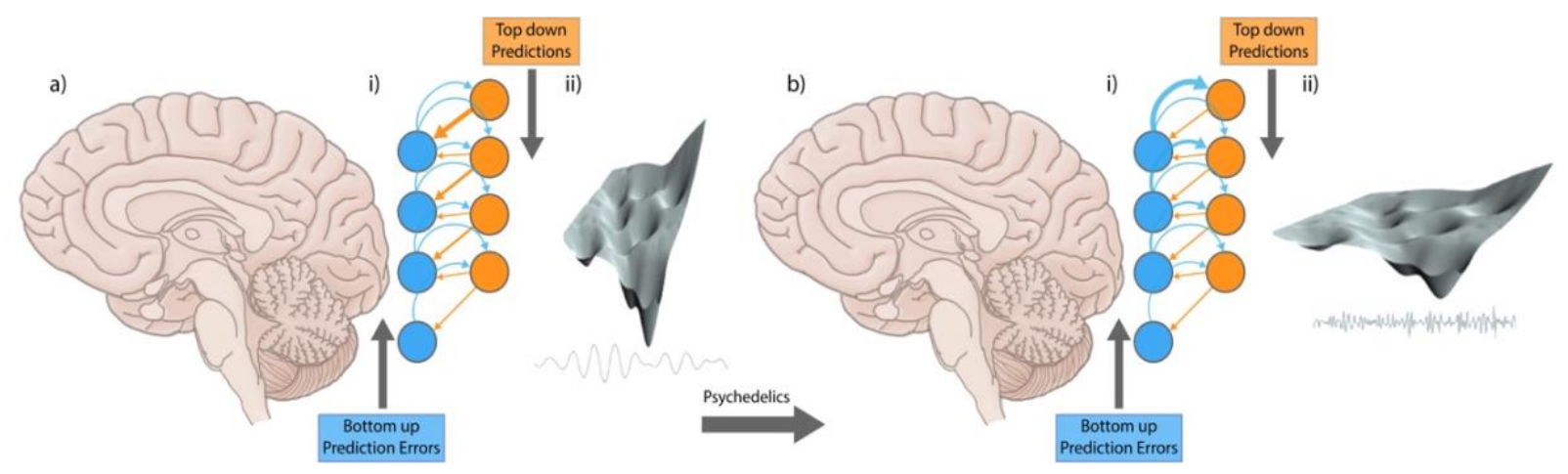

Figure 7. Panel a) i) Sensory input is compared with top-down predictions to form prediction errors that are passed on to higher levels of the hierarchy to revise Bayesian beliefs. These beliefs or representations then supply top-down predictions, which resolve the prediction errors at the lower level. This process is repeated to minimise the prediction error at each level. The predictive coding hierarchy tries to construct the best top-down explanation for bottom-up sensory input at each level of the hierarchy. Panel b) i) Psychedelics are thought to reduce precision and flatten the energy landscape of beliefs generated in high levels of the hierarchy supporting self-related beliefs, thereby producing the dissolving of self-related priors (i.e., ego dissolution). Panel a) ii) \& b) ii) Dissolution of precision of high-level priors flatten the curvature of the free energy landscape enabling neural dynamics to escape their local minima or basins of attraction, allowing greater attention to the sensory input and prediction errors (computationally expressed as a free energy landscape). The cognitive-therapeutic result of ego dissolution is the reduced precision or commitment to higher level beliefs in the high levels of the hierarchy that affords an opportunity to explore a landscape of alternative hypotheses of the causes of sensory impressions and the consequences of self-initiated actions. Change to these explanations can be therapeutic by enabling new ways to make sense of the world and lived exchanges with it. This notion of free energy landscapes is endorsed by empirical studies of electrical physiological responses and functional anatomy (Bastos et al., 2012). Borrowed from (R. L. Carhart-Harris \& Friston, 2019).

\section{Future research priorities}

Neuroimaging research provides a basis for interpreting psychedelic effects on consciousness and lasting behavioural outcomes. As has been described above, psychedelic changes span the hierarchical organisation of the brain-from binding to serotonergic receptors, to the downstream effects of oscillatory patterns on specialised regions and network connectivity. Measurement of correlates of psychedelic changes to neurons that form brain connectivity can contribute to our understanding of the biological underpinnings of psychedelic subjective 
effects. Analysis methods such as measuring the cytoarchitecture of the brain's hub regions may be a focus of future research. Cytoarchitecture research suggests that rich links between brain hubs can be explained by genetic factors while peripheral links are better explained by environmental influences (Fulcher \& Fornito, 2016). The desegregation of FC from network pathways under psychedelics (Lord et al., 2018; Luppi et al., 2021; Varley et al., 2019) also demonstrates deviation of connectivity mediating rich links that is consistent with the observations of increased sensitivity to intrinsic and extrinsic perturbations (i.e., environmental influences) under psychedelics.

Hierarchical interactions are necessarily directed (i.e., bottom-up and top-down). Functional connectivity measures are blind to directed connectivity. This suggests that more focus should be placed on the analysis of effective connectivity changes under psychedelics. Correlationbased whole brain FC techniques can be complemented by the ability of DCM to infer the directed causes of neuronal responses. This technique is capable of parcellating the brain hierarchy and identifying the direction and strength of message passing across subcortical, and cortical levels. Crucially, unlike functional connectivity, the effective connectivity estimates supplied by DCM include intrinsic, self connections; namely, the intrinsic excitability of different regions or neuronal populations. This is important in terms of estimating the effective precision or synaptic gain of a population under a hierarchical predictive coding formulation. In short, DCM can link synaptic level mechanisms described in hierarchical predictive processing to the directed connectivity changes of region level neural substrates.

\section{Separating subjective effects from therapeutic outcomes}

The necessity of subjective effects — for therapeutic outcomes — remains a debate that can be informed by further neurobiological study. Most psychedelic research suggests the psychological aspect of experience is crucial to therapeutic outcomes (Yaden \& Griffiths, 2020). This position can be understood through the influence of serotonin on precision control 
and suggests psychedelics act upon the very mechanisms of sentience and attention which may lead to therapeutic outcomes. Furthermore, the efficacy of these mechanisms is argued to depend on the psychological mindset of individuals and the setting of administration, in order to successfully ameliorate ego resistance (suggested here to be a prerequisite to positively felt ego dissolution). However, despite these factors, some research suggests that the biological effects of psychedelics may be divorced from the subjective psychedelic experience, while retaining therapeutic properties (Olson, 2020).

The theory that psychedelic therapeutic effects depend upon psychosocial contributions will be falsified if equal efficacy in the same range of pathologies can be achieved without subjective effects. Such an outcome suggests that the psychedelic aspect of psychedelics may be superfluous. This appears unlikely in the face of reported therapeutic experiences and outcomes, such as in the case of end-of-life psilocybin treatments (Cosimano, 2014). Psychedelic mechanisms separated from their psychological component may have utility, although a more plausible outcome may be a reduced efficacy in pathologies currently recommended for psychedelic therapy or findings that suggest the efficacy of drugs derived from psychedelics in alternate domains of medicine. In these eventualities, it may be argued that a unique class of drug has been invented and that the biopsychosocial approach to classic psychedelic administration for therapeutic uses remains efficacious.

Future pharmacology studies are sure to test this hypothesis and determine the limits of separating subjective experience from biologically induced therapeutic benefits. Neuroimaging research can also contribute to this line of inquiry. Research at the macroscopic level of brain regions, networks and their integration can address this question by delineating precise mechanisms. For example, measuring the effective connectivity between brain regions influenced by the $5 \mathrm{HT} 2 \mathrm{AR}$ involved in longitudinal studies may indicate whether changes associated with regions involved in consciousness correlate with wellbeing. This may indicate how therapeutic effects depend on changes in brain connectivity associated to 
consciousness and their separability from subjective effects. Meanwhile, interest in the manipulation of psychedelic molecules and research building upon pre-treatments acting as selective inhibitors of subjective effects (Katrin H. Preller et al., 2019; Quednow et al., 2012; F. X. Vollenweider, Vollenweider-Scherpenhuyzen, Babler, Vogel, \& Hell, 1998) will likely provide stronger understanding of the reliance of therapeutic outcomes upon subjective effects.

\section{Determinants of dose-response and behavioural measures of ego dissolution}

Uncertainty exists whether ego dissolution involves a gradual onset or a binary (i.e., all or nothing) experience. Evidence suggesting the binary (i.e., nonlinear) dose-response hypothesis is supported by PET investigation of plasma psilocin occupancy (the metabolite of psilocybin) and the reported subjective intensity of experience (Madsen et al., 2019). However visual schematics illustrating subjective responses of oceanic boundlessness (i.e., ego dissolution) counter this assertion by providing evidence of a gradual onset, see Figure 8 (Hirschfeld \& Schmidt, 2020).

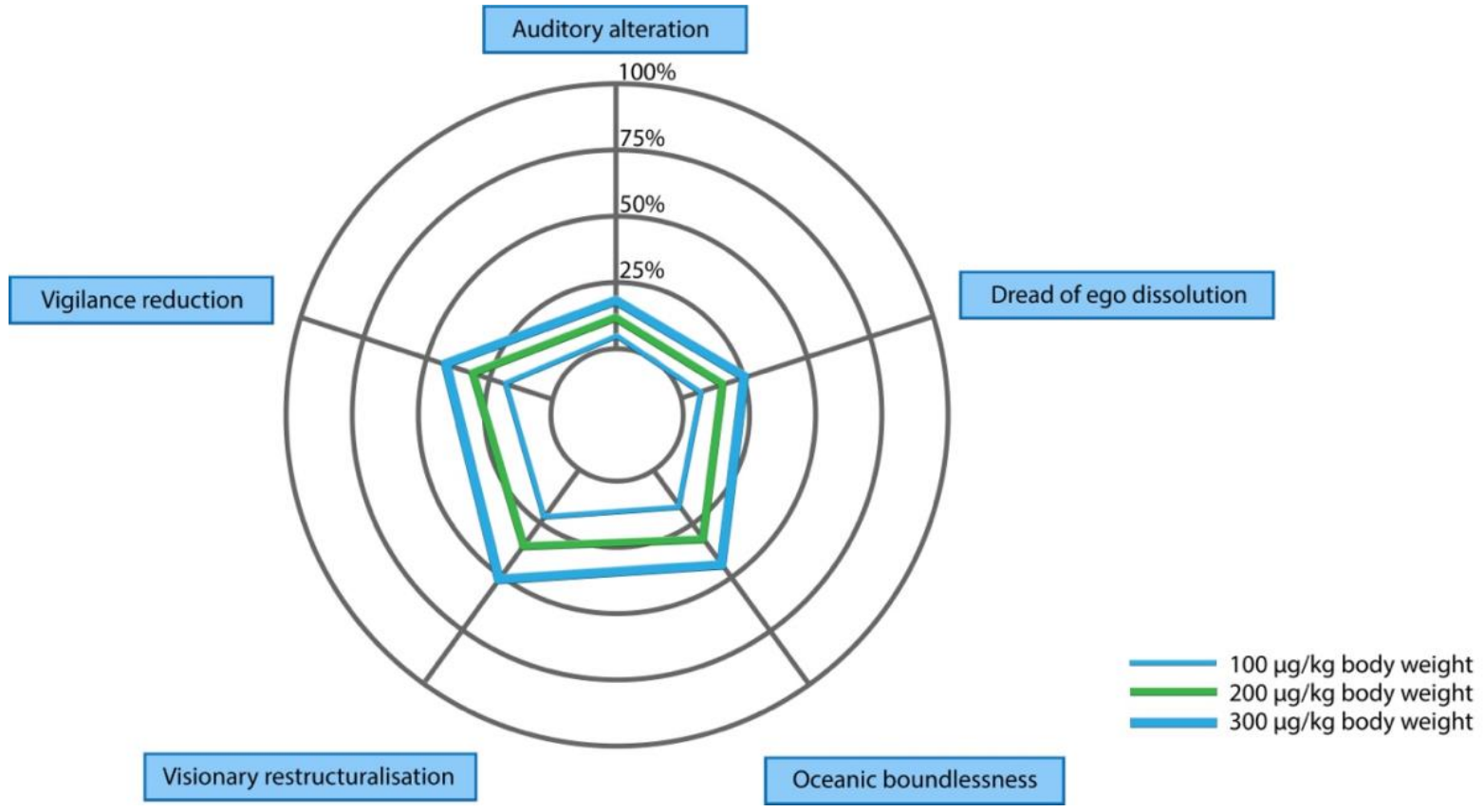

Figure 8. Ego dissolution rating by body weight adjusted psilocybin dose, adapted from Hirschfeld \& Schmidt, 2020 review of psilocybin studies using the 5D-ASC (5 dimensions of altered states of consciousness scale). Psilocybin doses assigned by varying body weight 
suggest ego dissolution (Oceanic boundlessness) may be amplified in a linear dosedependent manner (i.e., gradual) (Hirschfeld \& Schmidt, 2020).

Time dependent connectivity mechanisms of ego dissolution may be helpful to answer the dose-response question and have been used in the work of Preller et. al., 2020 (Katrin H. Preller et al., 2020). However, this study used a body-weight dose equivalent to $14 \mathrm{mg} / 70 \mathrm{~kg}$ while the standard "full" dose used in therapeutic trials is typically $25 \mathrm{mg}$. Sequential time dependent scanning and behavioural measures across high dose onset may require psychedelic experienced participants and enable better measurement of the correspondence between connectivity changes and the onset of ego dissolution. Follow up analysis may then observe whether a transition in brain connectivity accompanies reports of ego dissolution during brain imaging.

A related controversy pertains to psychedelic doses adjusted by body weight. Weight adjusted doses have been the standard form of dose administration across neuroimaging trials: however, recent evidence suggests no influence of body weight on subjective effects of psilocybin (Garcia-Romeu, Barrett, Carbonaro, Johnson, \& Griffiths, 2021). This research also marks the need for greater understanding of the mechanisms controlling the relationship between dose and response. Moreover, subjective responses can vary significantly following a standard or weight adjusted dose of a psychedelic (Katrin H. Preller et al., 2019). Several approaches to the dose-response question may be entertained. For example, greater understanding of relationship between subjective effects and the density and distribution of serotonin receptors may be informative. Measures of the volume and functional connectivity profiles of individual brain structures may also predict the dose-response relationship and warrants future investigation. For example, baseline global connectivity may be associated to subjective responses to psilocybin (Katrin H. Preller et al., 2020) and intensity of ego dissolution has been linked to a low diversity of connections in the anterior parahippocampus (Alexander V. Lebedev et al., 2015). Structural predictors of dose responses are also intriguing, following evidence that psychedelics can influence particular region volumes in long 
term users (Carlos Bouso et al., 2015) and rACC thickness can predict emotional subjective effects (Lewis et al., 2020). To date, no method has been established to predict dose-response relationships in psychedelic naive participants. Neuroimaging studies sensitive to associations between the density and distribution of receptors, region structural volume, measures of connectivity and the subjective response of participants may be valuable in determining predictors of dose-responses and enable the tailoring of doses for clinical applications. Posthoc analyses can also contribute to answering the dose-response question by determining how effective connectivity pathways between key brain regions and hierarchical strength (calculated by summing efferent and afferent region connectivity) (Zhou et al., 2018) between networks predict aspects of the experience reported on behavioural measures. Understanding the connectivity mechanisms of the dose-response relationship may provide biomarkers that determine the optimal dose in therapeutic contexts and, in turn, identify the mechanisms of subjective effects and ego dissolution.

Furthermore, improvements in existing behavioural measures administered after psychedelic ingestion are crucial to advancing research. At present, aspects of the psychedelic experience such as ego dissolution are lumped into a single measure of changes to sense of self and the relationship between subject and object. However neuroscientific and consciousness research suggest that the self can be dichotomised into different aspects related to distinct networks, such as the SN (i.e., minimal self) and DMN (i.e., narrative self). In associating connectivity patterns to ego dissolution, it may be essential to implement subjective measures sensitive to discriminations between minimal and narrative aspects of the self. These independent behavioural measures may then be associated with underlying connectivity and inform existing theories of SN and DMN contributions to ego dissolution. This will help determine network-dependent changes to consciousness under psychedelics with greater accuracy. 


\section{Comparative research}

Our knowledge of psychedelics may also benefit from protocols that compare connectivity changes observed across different classic psychedelics and a variety of other altered states of consciousness including psychosis and meditation (or even sleep). Parallels between the early stages of psychosis and psychedelic reports was one of the first insights into the nature of psychedelic induced conscious states. However, this parallel may have been biased by reports of negatively felt ego dissolution that was more likely in studies which did not appreciate the importance of supportive set and settings (Robin L. Carhart-Harris et al., 2018). Findings from these studies were more likely to resemble psychotic disturbances. Appreciation of the influence of context-on the valence of psychedelic experiences- motivates further study of both set and setting. Research testing the influence of factors contributing to the administration settings could manipulate imagery, lighting, sound, interactions with stimuli (e.g., walks in nature or experiences in virtual reality) and whether the administration of psychedelics occurs in an individual or group setting. Mindset may also be manipulated by controlling for subjects with specific characteristics, dispositions and training. The importance of mindset can also be demonstrated by three key manners in which psychedelic experiences differ from experiences of psychosis. These include consent that is given prior to administration, targeted use of psychedelic experience that may involve an intention for administration, and the temporary duration of the psychedelic experience. Provision and assurance of these factors are important contextual determinants expected to promote positively felt loss of self that differentiates psychedelic states from psychosis. Moreover, preparing mindset may influence the organised coherence of dysregulated and disintegrated connectivity under psychedelics (Atasoy et al., 2017). This hypothesis can be tested by testing for correlations between connectivity and participants' behavioural reports of positively felt ego dissolution (i.e., oceanic boundlessness on the 5D-ASC scale) and dread of ego dissolution (i.e., dread of ego dissolution on the 5D-ASC scale). Between subject studies can also be used to identify connectivity mechanism underlying specific facets of experience such as 
blissful state and sense of unity (R. L. Carhart-Harris, 2018b) that may demonstrate unique forms of coherence or stability of brain states (Atasoy et al., 2017) during ego dissolution.

The importance of supporting mindset factors speaks to the value of comparative research between meditation and psychedelics. Many authors have highlighted similarities and differences between meditation and psychedelics that provide a basis for future neuroimaging studies. The utility of meditation as an adjunct to psychedelic therapy is supported by research that found nominal anxiety in meditators (Smigielski, Kometer, et al., 2019) and enhanced depth of meditation (Smigielski, Scheidegger, et al., 2019). This synergistic approach-also possible with music (Kaelen et al., 2018)—can be used to evaluate the benefits to behavioural outcomes and accompanying connectivity changes. The importance of integrating meditation in psychedelic study design is further highlighted by evidence that the quality of psychedelic experiences may determine therapeutic outcomes (Roseman, Nutt, et al., 2018). Future study designs could integrate meditation training prior to psychedelic use to prepare participants, enhanced positive ego dissolution and measure connectivity changes associated to the inclusion of meditation. They may also test the effect of meditation programs after the psychedelic experience as a form of integration to cement hypothesised neuroplastic changes that may underwrite behavioural change. Some work in this direction (previously discussed) has been undertaken by Griffiths et. al., 2018 and Smigielski et. al., 2019: however, relatively less focus has been placed upon programs implemented post-hoc. In summary, equipping neuroimaging protocols with groups assigned to meditation programs can help identify the connectivity differences based on meditation and psychedelic synergy.

\section{Consciousness research}

Psychedelics show value as a tool for a neuroscientific understanding of consciousness. The search for the neural correlates of ego dissolution require the minimally sufficient neural prerequisites of consciousness to be identified, in order to claim the neural activity correlated

with consciousness has been determined (Chalmers, 2000). Ego dissolution may not 
encompass the minimally sufficient neural prerequisites of consciousness, because some important aspects of consciousness are retained in psychedelic experiences. However, they may still reveal some neural prerequisites of consciousness if change to connectivity induced by psychedelics is found to operate exclusively in conscious states (Kalat, 2014; Millière, 2017). Future neuroimaging research could identify neural mechanisms underlying psychedelic subjective effects and states of ego dissolution that are distinct from other states of transcendence, such as meditation.

\section{Plasticity}

Apart from understanding the brain connectivity that underlies ego dissolution and its potential to inform our understanding of consciousness, the related idea of psychedelic-induced neuroplasticity proposes a fruitful avenue for future psychedelic research. The notion that psychedelics leave the brain in a more plastic (i.e., malleable) state has a substantial body of evidence and similarly abundant evidence suggests lasting behavioural changes. However, further research is required to identify the synaptic changes and ensuing effects on effective connectivity in humans and determine what biological changes exist after psychedelics have been fully eliminated. Understanding this could elucidate the synaptic mechanisms supporting lasting impressions on consciousness that underwrite therapeutic benefits. One line of inquiry-connecting neural mechanisms to lasting behavioural change-is measures of entropy induced by psychedelics that have been related to personality change (A. V. Lebedev et al., 2016; Liechti, 2017). Psychedelic induced entropy may relate to increased openness, a personality trait connected to schizotypy. Schizotypy describes an imaginative state of mind that, in extreme presentations, shares similarities with psychosis. However, this facet of personality is also associated to beneficial outcomes (Mohr \& Claridge, 2015; Schultze-Lutter, Nenadic, \& Grant, 2019). 
Neuroimaging research can clarify the link between connectivity and behaviour (associated with openness) by estimating connectivity before and after psychedelic use. This may provide an indication of neuroplastic changes in the brain. Similar efforts already exist showing evidence of connectivity changes in the AMG lasting up to one month (F. S. Barrett et al., 2020) and mPFC-PCC in meditators performing meditation in the scanner post administration of psilocybin (Smigielski, Scheidegger, et al., 2019). These findings suggest underlying neuroplastic mechanisms may account for lasting connectivity changes and behavioural outcomes. Thorough investigations of neural plasticity across the brain remain a direction of future neuroimaging research and may discover important clinical applications such as advancing the understanding of the pathophysiology and prevention of neurodegeneration. These future studies can study the influence of psychedelic on regional connectivity changes over time after administration. This research can also be refined by investigating how contextual determinants and psychological support influence connectivity changes indicative of plasticity-and how biological processes may translate into psychological growth. Optimised integration programs following psychedelics that cement insights developed during ego dissolution may capitalise on hypothesised neuroplastic benefits of reduced ego resistance. Measuring connectivity differences following effective psychedelic integration can advance our ability to discern and alter brain connectivity for wellbeing.

\section{Conclusions}

Historical records indicate the use of classic psychedelics to alter consciousness and manifest insight has existed in cultures around the world since antiquity. Their popularisation in Western culture during the mid- $20^{\text {th }}$ century affected the thoughts and feeling of many who used them, leading to a revision of socio-political beliefs. The backlash of this threat to hierarchicalpolitical authority was the sensationalism of fears and legislation that cast shade over psychedelic research until the turn of the millennium. Motivated by academic psychiatry, researchers revisited the signs of potential indicated by early psychedelic research. Their 
findings challenged the legislation of psychedelics as substances with no therapeutic or scientific value. Since this ground-breaking research, which demonstrated the efficacy of psychedelics, research into their therapeutic applications has accelerated, and consciousness research has followed to understand the underlying mechanisms of therapeutic outcomes and subjective effects.

Psychedelic changes to consciousness are now understood to derive from the pharmacological action on serotonergic 5HT2A receptors. Agonist activity results in entropic changes influencing the synaptic gain of neural populations, which affects precision control, and which underwrites inferences about the causes of sensation and consequences of actions. Understanding the effect of serotonergic activity on whole brain connectivity poses significant challenges. Different brain regions have specialised functions, and these regions are functionally integrated into networks that serve roles underlying conscious processes. Applying an understanding of regional and network-level function in consciousness to psychedelic subjective effects, underwritten by changes to oscillatory rhythms (i.e., connectivity) within and between brain substrates, is a promising direction for future psychedelics neuroimaging research. Here, we reviewed regional and network-level analyses of psychedelic changes to brain connectivity, which identified mechanisms that may contribute to ego dissolution and therapeutic effects.

Neuroimaging endeavours are required to address a range of questions probing the nature of psychedelics. Answers to these questions depend on (repeated) neuroimaging experiments that tests hypotheses of connectivity changes, and advancement of behavioural measures. We will then be able to more precisely discriminate features of subjective experience associated with brain connectivity and provide further empirical evidence for unifying frameworks such as hierarchical predictive coding. 
Hierarchical predictive coding provides a biologically plausible computational model of sentience and serves as a model of neuronal function that is an essential building block to understanding neural processes. Hierarchical predictive coding explains serotonergic synaptic mechanism's role in making sense of the world through top-down predictions (or inferences) and bottom-up prediction errors (that drive belief updating). These neural mechanisms offer a means of synthesising psychological and neuroscientific interpretation of psychedelic neuroimaging observations. Understanding the link between psychology and neurology is crucial, following behavioural evidence that reaction to a dose of a psychedelic substance depends upon the environment in which is it is taken and mindset of the subject. This evidence indicates the importance of understanding the role of biopsychosocial factors. These factors may also be crucial to predicting and supporting therapeutic efficacy. Future therapeutic research is encouraged to support positively felt ego dissolution by cultivating reduced ego resistance. This may entail manipulating and optimising a subject's preparation, the administrative setting, and integration programs designed to cement psychedelic insights into lasting wellbeing. Further understanding of the interactions of biopsychosocial influences of serotonergic neural mechanisms can be aided by neuroimaging analyses that observe differences between the brain connectivity of successful and unsuccessful psychedelic interventions. This may help explain neural mechanisms underlying wellbeing, hypothesised to be underwritten by neuroplasticity, and suggests the important contribution of psychedelic neuroimaging to psychiatry.

Hierarchical predictive coding also offers a framework for understanding the neural mechanisms of consciousness. Psychedelics provide hierarchical predictive coding with a means of testing its modelling of sentient behaviour and advancing the understanding of serotonergic predictive processes in consciousness and attention. The alteration of these synaptic processes by psychedelics results in subjective effects, argued here to be an essential component of therapeutic outcomes. Hierarchical predictive coding explains subjective and therapeutic mechanisms at the neural level which suggests that further 
investigations that can quantify the changes to the hierarchical organisation of connectivity under psychedelics are essential. Dynamic causal modelling offers a method of testing changes to hierarchically organised connectivity and can be synthesised with hierarchical predictive coding to help model change to brain connectivity involved in consciousness and attention. Applying the understanding of predictive processes may also help synthesise interpretations across various neuroimaging analysis methods that describe macro and micro scale changes to the brain-and their association with subjective effects.

Through psychedelic alteration of serotonergic receptors, the hierarchical relationship between top-down associative processes and bottom-up sensations are collapsed. This collapse inhibits associative functions of regions and networks involved in conscious beliefs and perceptions and enables a landscape in which bottom-up influences can update beliefs about interactions with the world. This temporary collapse is reflected in the breakdown of self, evidenced by ego dissolution, and the updating of beliefs is demonstrated in therapeutic insights leading to changes in patterns of thought and perspective.

Interestingly, the introduction of psychedelics to western culture reflects a similar process of reidentification through challenge to hierarchical authority of top-down beliefs from political leadership and bottom-up grassroots influences. This suggests a common effect of psychedelics across neurology and sociology; in which boundaries limiting perception are broken. Moreover, it indicates a reflection between the hierarchical modelling of the brain and social organisation. Surmising that the experience of ego dissolution exists at the centre of the psychedelic experience and that its neural mechanisms may be close to the roots of the subject-object dichotomy may explain why phenomenological encounters with this experience can be intimate and fundamentally change self-identification and interactions with the world. Continued investigation of the neural mechanisms of ego dissolution and the dichotomy of separation between the self and the world may advance scientific understanding of sentience. 
The value of psychedelics may then, under appropriate conditions, be their power to catalyse meaningful philosophical and scientific understanding of the "u" (i.e., self) in the universe.

\section{Acknowledgements}

AR is funded by the Australian Research Council (Refs: DE170100128 \& DP200100757) and Australian National Health and Medical Research Council Investigator Grant (Ref: 1194910). AR is a CIFAR Azrieli Global Scholar in the Brain, Mind \& Consciousness Program. AR and KF are affiliated with The Wellcome Centre for Human Neuroimaging supported by core funding from Wellcome [203147/Z/16/Z].

\section{References}

Adams, R., Stephan, K., Brown, H., Frith, C., \& Friston, K. (2013). The Computational Anatomy of Psychosis. Frontiers in Psychiatry, 4(47). doi:10.3389/fpsyt.2013.00047

Adolphs, R. (2010). What does the amygdala contribute to social cognition? Annals of the New York Academy of Sciences, 1191(1), 42-61. doi:https://doi.org/10.1111/j.17496632.2010.05445.x

Ainley, V., Apps, M. A., Fotopoulou, A., \& Tsakiris, M. (2016). 'Bodily precision': a predictive coding account of individual differences in interoceptive accuracy. Philos Trans R Soc Lond B Biol Sci, 371(1708). doi:10.1098/rstb.2016.0003

Ainley, V., Tajadura-Jiménez, A., Fotopoulou, A., \& Tsakiris, M. (2012). Looking into myself: changes in interoceptive sensitivity during mirror self-observation. Psychophysiology, 49(11), 3936-3946.

Almgren, H., Van de Steen, F., Razi, A., Friston, K., \& Marinazzo, D. (2020). The effect of global signal regression on DCM estimates of noise and effective connectivity from resting state fMRI. NeuroImage, 208, 116435. doi:https://doi.org/10.1016/j.neuroimage.2019.116435

Aminoff, E. M., Kveraga, K., \& Bar, M. (2013). The role of the parahippocampal cortex in cognition. Trends Cogn Sci, 17(8), 379-390. doi:10.1016/j.tics.2013.06.009

Anand, K. S., \& Dhikav, V. (2012). Hippocampus in health and disease: An overview. Annals of Indian Academy of Neurology, 15(4), 239-246. doi:10.4103/09722327.104323

Andrade, R. (2011). Serotonergic regulation of neuronal excitability in the prefrontal cortex. Neuropharmacology, 61(3), 382-386. doi:10.1016/j.neuropharm.2011.01.015

Andrews-Hanna, J. R., Reidler, J. S., Sepulcre, J., Poulin, R., \& Buckner, R. L. (2010). Functional-anatomic fractionation of the brain's default network. Neuron, 65(4), 550562. doi:10.1016/j.neuron.2010.02.005

Andrews-Hanna, J. R., Smallwood, J., \& Spreng, R. N. (2014). The default network and selfgenerated thought: component processes, dynamic control, and clinical relevance. 
Annals of the New York Academy of Sciences, 1316(1), 29-52.

doi:10.1111/nyas.12360

Aquino, K. M., Fulcher, B. D., Parkes, L., Sabaroedin, K., \& Fornito, A. (2020). Identifying and removing widespread signal deflections from fMRI data: Rethinking the global signal regression problem. NeuroImage, 212, 116614. doi:10.1016/j.neuroimage.2020.116614

Arieli, A., Sterkin, A., Grinvald, A., \& Aertsen, A. (1996). Dynamics of ongoing activity: explanation of the large variability in evoked cortical responses. Science, 273(5283), 1868-1871. doi:10.1126/science.273.5283.1868

Asemi, A., Ramaseshan, K., Burgess, A., Diwadkar, V. A., \& Bressler, S. L. (2015). Dorsal anterior cingulate cortex modulates supplementary motor area in coordinated unimanual motor behavior. Frontiers in Human Neuroscience, 9, 309-309. doi:10.3389/fnhum.2015.00309

Atasoy, S., Roseman, L., Kaelen, M., Kringelbach, M. L., Deco, G., \& Carhart-Harris, R. L. (2017). Connectome-harmonic decomposition of human brain activity reveals dynamical repertoire re-organization under LSD. Scientific Reports, 7. doi:10.1038/s41598-017-17546-0

Auger, S. D., \& Maguire, E. A. (2018). Retrosplenial Cortex Indexes Stability beyond the Spatial Domain. The Journal of Neuroscience, 38(6), 1472. doi:10.1523/JNEUROSCI.2602-17.2017

Auger, S. D., Mullally, S. L., \& Maguire, E. A. (2012). Retrosplenial Cortex Codes for Permanent Landmarks. Plos One, 7(8), e43620. doi:10.1371/journal.pone.0043620

Averbeck, B. B., \& Seo, M. (2008). The Statistical Neuroanatomy of Frontal Networks in the Macaque. PLOS Computational Biology, 4(4), e1000050. doi:10.1371/journal.pcbi.1000050

Bak, P., Tang, C., \& Wiesenfeld, K. (1987). Bak, P., Tang, C. \& Wiesenfeld, K. Selforganized criticality: An explanation of 1/f noise. Phys. Rev. Lett. 59, 381-384. Physical Review Letters, 59, 381-384. doi:10.1103/PhysRevLett.59.381

Barnett, L., Muthukumaraswamy, S. D., Carhart-Harris, R. L., \& Seth, A. K. (2019). Decreased Directed Functional Connectivity in the Psychedelic State. bioRxiv, 703660. doi: $10.1101 / 703660$

Barrett, F. S., Doss, M. K., Sepeda, N. D., Pekar, J. J., \& Griffiths, R. R. (2020). Emotions and brain function are altered up to one month after a single high dose of psilocybin. Sci Rep, 10(1), 2214. doi:10.1038/s41598-020-59282-y

Barrett, F. S., Krimmel, S. R., Griffiths, R. R., Seminowicz, D. A., \& Mathur, B. N. (2020). Psilocybin acutely alters the functional connectivity of the claustrum with brain networks that support perception, memory, and attention. NeuroImage, 218, 116980. doi:https://doi.org/10.1016/j.neuroimage.2020.116980

Barrett, F. S., Robbins, H., Smooke, D., Brown, J. L., \& Griffiths, R. R. (2017). Qualitative and Quantitative Features of Music Reported to Support Peak Mystical Experiences during Psychedelic Therapy Sessions. Frontiers in Psychology, 8(1238). doi:10.3389/fpsyg.2017.01238

Barron, D. S., Tandon, N., Lancaster, J. L., \& Fox, P. T. (2014). Thalamic structural connectivity in medial temporal lobe epilepsy. Epilepsia, 55(6), e50-55. doi:10.1111/epi.12637

Bastos, A. M., Usrey, W. M., Adams, R. A., Mangun, G. R., Fries, P., \& Friston, K. J. (2012). Canonical microcircuits for predictive coding. Neuron, 76(4), 695-711. doi:10.1016/j.neuron.2012.10.038

Bedi, G., Hyman, D., \& de Wit, H. (2010). Is ecstasy an "empathogen"? Effects of $\pm 3,4-$ methylenedioxymethamphetamine on prosocial feelings and identification of 
emotional states in others. Biological Psychiatry, 68(12), 1134-1140.

doi:10.1016/j.biopsych.2010.08.003

Behrendt, R. P., \& Young, C. (2004). Hallucinations in schizophrenia, sensory impairment, and brain disease: a unifying model. Behav Brain Sci, 27(6), 771-787; discussion 787830. doi:10.1017/s0140525x04000184

Beliveau, V., Ganz, M., Feng, L., Ozenne, B., Højgaard, L., Fisher, P. M., . . Knudsen, G. M. (2017). A High-Resolution \&lt;em\&gt;In Vivo\&lt;/em\&gt; Atlas of the Human Brain\&\#039;s Serotonin System. The Journal of Neuroscience, 37(1), 120. doi:10.1523/JNEUROSCI.2830-16.2016

Bestmann, S., Harrison, L. M., Blankenburg, F., Mars, R. B., Haggard, P., Friston, K. J., \& Rothwell, J. C. (2008). Influence of uncertainty and surprise on human corticospinal excitability during preparation for action. Curr Biol, 18(10), 775-780. doi:10.1016/j.cub.2008.04.051

Betti, V., Della Penna, S., de Pasquale, F., \& Corbetta, M. (2020). Spontaneous Beta Band Rhythms in the Predictive Coding of Natural Stimuli. The Neuroscientist, 27(2), 184201. doi: $10.1177 / 1073858420928988$

Blanke, O., \& Arzy, S. (2005). The out-of-body experience: disturbed self-processing at the temporo-parietal junction. Neuroscientist, 11(1), 16-24. doi:10.1177/1073858404270885

Blanke, O., \& Metzinger, T. (2009). Full-body illusions and minimal phenomenal selfhood. Trends Cogn Sci, 13(1), 7-13. doi:10.1016/j.tics.2008.10.003

Bogenschutz, M. P., Forcehimes, A. A., Pommy, J. A., Wilcox, C. E., Barbosa, P. C., \& Strassman, R. J. (2015). Psilocybin-assisted treatment for alcohol dependence: a proof-of-concept study. J Psychopharmacol, 29(3), 289-299. doi:10.1177/0269881114565144

Bolton, T., Wotruba, D., Buechler, R., Theodoridou, A., Michels, L., Kollias, S., . . Van De Ville, D. (2020). Triple Network Model Dynamically Revisited: Lower Salience Network State Switching in Pre-psychosis. Frontiers in Physiology, 11. doi:10.3389/fphys.2020.00066

Boly, M., Phillips, C., Tshibanda, L., Vanhaudenhuyse, A., Schabus, M., Dang-Vu, T. T., .. . Laureys, S. (2008). Intrinsic brain activity in altered states of consciousness: how conscious is the default mode of brain function? Ann N Y Acad Sci, 1129, 119-129. doi:10.1196/annals.1417.015

Bonnelle, V., Ham, T. E., Leech, R., Kinnunen, K. M., Mehta, M. A., Greenwood, R. J., \& Sharp, D. J. (2012). Salience network integrity predicts default mode network function after traumatic brain injury. Proc Natl Acad Sci U S A, 109(12), 4690-4695. doi:10.1073/pnas.1113455109

Bouso, J. C., Dos Santos, R. G., Alcazar-Corcoles, M. A., \& Hallak, J. E. C. (2018). Serotonergic psychedelics and personality: A systematic review of contemporary research. Neurosci Biobehav Rev, 87, 118-132. doi:10.1016/j.neubiorev.2018.02.004

Bréchet, L., Grivaz, P., Gauthier, B., \& Blanke, O. (2018). Common Recruitment of Angular Gyrus in Episodic Autobiographical Memory and Bodily Self-Consciousness. Frontiers in Behavioral Neuroscience, 12(270). doi:10.3389/fnbeh.2018.00270

Brewer, J. A., Worhunsky, P. D., Gray, J. R., Tang, Y.-Y., Weber, J., \& Kober, H. (2011). Meditation experience is associated with differences in default mode network activity and connectivity. Proceedings of the National Academy of Sciences, 108(50), 2025420259. doi:10.1073/pnas.1112029108

Brown, H., Adams, R. A., Parees, I., Edwards, M., \& Friston, K. (2013). Active inference, sensory attenuation and illusions. Cognitive processing, 14(4), 411-427. doi:10.1007/s10339-013-0571-3 
Brown, S. P., Mathur, B. N., Olsen, S. R., Luppi, P.-H., Bickford, M. E., \& Citri, A. (2017). New Breakthroughs in Understanding the Role of Functional Interactions between the Neocortex and the Claustrum. The Journal of neuroscience : the official journal of the Society for Neuroscience, 37(45), 10877-10881. doi:10.1523/JNEUROSCI.183717.2017

Buckner, R. L., Andrews-Hanna, J. R., \& Schacter, D. L. (2008). The brain's default network: anatomy, function, and relevance to disease. Ann N Y Acad Sci, 1124, 1-38. doi:10.1196/annals.1440.011

Busch, N. A., Dubois, J., \& VanRullen, R. (2009). The Phase of Ongoing EEG Oscillations Predicts Visual Perception. The Journal of Neuroscience, 29(24), 7869-7876. doi:10.1523/jneurosci.0113-09.2009

Buzsáki, G., \& Llinás, R. (2017). Space and time in the brain. Science (New York, N.Y.), 358(6362), 482-485. doi:10.1126/science.aan8869

Byock, I. (2018). Taking Psychedelics Seriously. Journal of Palliative Medicine, 21(4), 417421. doi:10.1089/jpm.2017.0684

Calhoun, V. (2011). Intrinsic Functional and Structural Networks in Schizophrenia, Bipolar Disorder, and Healthy Controls. Paper presented at the Biological Psychiatry.

Carbonaro, T. M., Bradstreet, M. P., Barrett, F. S., MacLean, K. A., Jesse, R., Johnson, M. W., \& Griffiths, R. R. (2016). Survey study of challenging experiences after ingesting psilocybin mushrooms: Acute and enduring positive and negative consequences. Journal of Psychopharmacology, 30(12), 1268-1278. doi:10.1177/0269881116662634

Carhart-Harris, R. (2007). Waves of the Unconscious: The Neurophysiology of $<\mathrm{i}>$ Dreamlike $</ \mathrm{i}>$ Phenomena and Its Implications for the Psychodynamic Model of the Mind. Neuropsychoanalysis, 9(2), 183-211. doi:10.1080/15294145.2007.10773557

Carhart-Harris, R., Kaelen, M., \& Nutt, D. (2014). How do hallucinogens work on the brain? Psychologist, 27(9), 662-665. Retrieved from <Go to ISI>://WOS:000341172000027

Carhart-Harris, R. L. (2018a). The entropic brain - revisited. Neuropharmacology, 142, 167178. doi:10.1016/j.neuropharm.2018.03.010

Carhart-Harris, R. L. (2018b). How do psychedelics work? Current Opinion in Psychiatry, 1. doi:10.1097/YCO.0000000000000467

Carhart-Harris, R. L., Bolstridge, M., Rucker, J., Day, C. M. J., Erritzoe, D., Kaelen, M., . . . Nutt, D. J. (2016). Psilocybin with psychological support for treatment-resistant depression: an open-label feasibility study. Lancet Psychiatry, 3(7), 619-627. doi:10.1016/s2215-0366(16)30065-7

Carhart-Harris, R. L., Erritzoe, D., Haijen, E., Kaelen, M., \& Watts, R. (2018). Psychedelics and connectedness. Psychopharmacology, 235(2), 547-550. doi:10.1007/s00213-0174701-y

Carhart-Harris, R. L., Erritzoe, D., Williams, T., Stone, J. M., Reed, L. J., Colasanti, A., . . Nutt, D. J. (2012). Neural correlates of the psychedelic state as determined by fMRI studies with psilocybin. Proceedings of the National Academy of Sciences, 109(6), 2138-2143. doi:10.1073/pnas.1119598109

Carhart-Harris, R. L., Erritzoe, D., Williams, T., Stone, J. M., Reed, L. J., Colasanti, A., . . Nutt, D. J. (2012). Neural correlates of the psychedelic state as determined by fMRI studies with psilocybin. Proceedings of the National Academy of Sciences of the United States of America, 109(6), 2138-2143. Retrieved from http://www.jstor.org.ezproxy.lib.monash.edu.au/stable/41477085

https://www.ncbi.nlm.nih.gov/pmc/articles/PMC3277566/pdf/pnas.201119598.pdf 
Carhart-Harris, R. L., \& Friston, K. J. (2010). The default-mode, ego-functions and freeenergy: a neurobiological account of Freudian ideas. Brain, 133(Pt 4), 1265-1283. doi:10.1093/brain/awq010

Carhart-Harris, R. L., \& Friston, K. J. (2019). REBUS and the Anarchic Brain: Toward a Unified Model of the Brain Action of Psychedelics. Pharmacological Reviews, 71(3), 316-344. doi:10.1124/pr.118.017160

Carhart-Harris, R. L., \& Goodwin, G. M. (2017). The Therapeutic Potential of Psychedelic Drugs: Past, Present, and Future. Neuropsychopharmacology : official publication of the American College of Neuropsychopharmacology, 42(11), 2105-2113. doi:10.1038/npp.2017.84

Carhart-Harris, R. L., Kaelen, M., Whalley, M. G., Bolstridge, M., Feilding, A., \& Nutt, D. J. (2015). LSD enhances suggestibility in healthy volunteers. Psychopharmacology, 232(4), 785-794. doi:10.1007/s00213-014-3714-z

Carhart-Harris, R. L., Leech, R., Erritzoe, D., Williams, T. M., Stone, J. M., Evans, J., . . Nutt, D. J. (2012). Functional Connectivity Measures After Psilocybin Inform a Novel Hypothesis of Early Psychosis. Schizophrenia Bulletin, 39(6), 1343-1351. doi:10.1093/schbul/sbs117

Carhart-Harris, R. L., Leech, R., Hellyer, P. J., Shanahan, M., Feilding, A., Tagliazucchi, E., . . Nutt, D. (2014). The entropic brain: a theory of conscious states informed by neuroimaging research with psychedelic drugs. Frontiers in Human Neuroscience, 8. doi:10.3389/fnhum.2014.00020

Carhart-Harris, R. L., Leech, R., Williams, T. M., Erritzoe, D., Abbasi, N., Bargiotas, T., . . . Nutt, D. J. (2012). Implications for psychedelic-assisted psychotherapy: functional magnetic resonance imaging study with psilocybin. British Journal of Psychiatry, 200(3), 238-244. doi:10.1192/bjp.bp.111.103309

Carhart-Harris, R. L., Muthukumaraswamy, S., Roseman, L., Kaelen, M., Droog, W., Murphy, K., . . Nutt, D. J. (2016). Neural correlates of the LSD experience revealed by multimodal neuroimaging. Proceedings of the National Academy of Sciences of the United States of America, 113(17), 4853-4858. Retrieved from https://www-jstororg.ezproxy.lib.monash.edu.au/stable/26469438

http://spiral.imperial.ac.uk/bitstream/10044/1/50366/5/PNAS-2016-Carhart-Harris-48538.pdf

Carhart-Harris, R. L., \& Nutt, D. J. (2017). Serotonin and brain function: a tale of two receptors. Journal of Psychopharmacology, 31(9), 1091-1120. doi:10.1177/0269881117725915

Carhart-Harris, R. L., Roseman, L., Bolstridge, M., Demetriou, L., Pannekoek, J. N., Wall, M. B., . . . Nutt, D. J. (2017). Psilocybin for treatment-resistant depression: fMRImeasured brain mechanisms. Scientific Reports, 7(1), 13187. doi:10.1038/s41598017-13282-7

Carhart-Harris, R. L., Roseman, L., Haijen, E., Erritzoe, D., Watts, R., Branchi, I., \& Kaelen, M. (2018). Psychedelics and the essential importance of context. Journal of Psychopharmacology, 32(7), 725-731. doi:10.1177/0269881118754710

Carlos Bouso, J., Palhano-Fontes, F., Rodriguez-Fornells, A., Ribeiro, S., Sanches, R., Crippa, J. A. S., . . Riba, J. (2015). Long-term use of psychedelic drugs is associated with differences in brain structure and personality in humans. European Neuropsychopharmacology, 25(4), 483-492. doi:10.1016/j.euroneuro.2015.01.008

Carod-Artal, F. J. (2015). Hallucinogenic drugs in pre-Columbian Mesoamerican cultures. Neurologia, 30(1), 42-49. doi:10.1016/j.nrl.2011.07.003

Chalmers, D. (2000). What Is a Neural Correlate of Consciousness. Neural Correlates of Consciousness. doi:10.1093/acprof:oso/9780195311105.003.0003 
Chand, G. B., Wu, J., Hajjar, I., \& Qiu, D. (2017). Interactions of the Salience Network and Its Subsystems with the Default-Mode and the Central-Executive Networks in Normal Aging and Mild Cognitive Impairment. Brain Connectivity, 7(7), 401-412. doi:10.1089/brain.2017.0509

Clark, A. (2013). Whatever next? Predictive brains, situated agents, and the future of cognitive science. Behav Brain Sci, 36(3), 181-204. doi:10.1017/s0140525x12000477

Cocchi, L., Gollo, L. L., Zalesky, A., \& Breakspear, M. (2017). Criticality in the brain: A synthesis of neurobiology, models and cognition. Progress in Neurobiology, 158, 132-152. doi:https://doi.org/10.1016/j.pneurobio.2017.07.002

Corbetta, M., \& Shulman, G. (2002). Control of Goal-Directed and Stimulus-Driven Attention in the Brain. Nature reviews. Neuroscience, 3, 201-215. doi:10.1038/nrn755

Cosimano, M. P. (2014). My Experience as a Guide in the Johns Hopkins Psilocybin Research Project. MAPS Bulletin, 24.

Cowan, W. M., Harter, D. H., \& Kandel, E. R. (2000). The emergence of modern neuroscience: some implications for neurology and psychiatry. Annu Rev Neurosci, 23, 343-391. doi:10.1146/annurev.neuro.23.1.343

Craig, A. D. (2009). How do you feel--now? The anterior insula and human awareness. Nat Rev Neurosci, 10(1), 59-70. doi:10.1038/nrn2555

Cummins, C., \& Lyke, J. (2013). Peak Experiences of Psilocybin Users and Non-Users. Journal of Psychoactive Drugs, 45(2), 189-194. doi:10.1080/02791072.2013.785855

Dahl, C. J., Lutz, A., \& Davidson, R. J. (2015). Reconstructing and deconstructing the self: cognitive mechanisms in meditation practice. Trends Cogn Sci, 19(9), 515-523. doi:10.1016/j.tics.2015.07.001

Davidson, R. J., Putnam, K. M., \& Larson, C. L. (2000). Dysfunction in the neural circuitry of emotion regulation--a possible prelude to violence. Science, 289(5479), 591-594. doi:10.1126/science.289.5479.591

Dehaene, S., \& Changeux, J.-P. (2011). Experimental and Theoretical Approaches to Conscious Processing. Neuron, 70(2), 200-227. doi:https://doi.org/10.1016/j.neuron.2011.03.018

Dehaene, S., \& Naccache, L. (2001). Towards a cognitive neuroscience of consciousness: basic evidence and a workspace framework. Cognition, 79(1-2), 1-37. doi:10.1016/s0010-0277(00)00123-2

Demertzi, A., Tagliazucchi, E., Dehaene, S., Deco, G., Barttfeld, P., Raimondo, F., . . Sitt, J. (2019). Human consciousness is supported by dynamic complex patterns of brain signal coordination. Science Advances, 5, eaat7603. doi:10.1126/sciadv.aat7603

Dillon, D. G., \& Pizzagalli, D. A. (2007). Inhibition of Action, Thought, and Emotion: A Selective Neurobiological Review. Applied \& preventive psychology : journal of the American Association of Applied and Preventive Psychology, 12(3), 99-114. doi:10.1016/j.appsy.2007.09.004

Dinis-Oliveira, R. J. (2017). Metabolism of psilocybin and psilocin: clinical and forensic toxicological relevance. Drug Metabolism Reviews, 49(1), 84-91. doi:10.1080/03602532.2016.1278228

Disner, S. G., Beevers, C. G., Haigh, E. A., \& Beck, A. T. (2011). Neural mechanisms of the cognitive model of depression. Nat Rev Neurosci, 12(8), 467-477. doi:10.1038/nrn3027

Dittrich, A. (1998). The standardized psychometric assessment of altered states of consciousness (ASCs) in humans. Pharmacopsychiatry, 31 Suppl 2, 80-84. doi:10.1055/s-2007-979351

Doll, A., Hölzel, B. K., Boucard, C. C., Wohlschläger, A. M., \& Sorg, C. (2015). Mindfulness is associated with intrinsic functional connectivity between default mode 
and salience networks. Frontiers in Human Neuroscience, 9, 461. doi:10.3389/fnhum.2015.00461

Donegan, N. H., Sanislow, C. A., Blumberg, H. P., Fulbright, R. K., Lacadie, C., Skudlarski, P., ... Wexler, B. E. (2003). Amygdala hyperreactivity in borderline personality disorder: implications for emotional dysregulation. Biol Psychiatry, 54(11), 12841293. doi:10.1016/s0006-3223(03)00636-X

Donoso, M., Collins, A. G., \& Koechlin, E. (2014). Human cognition. Foundations of human reasoning in the prefrontal cortex. Science, 344(6191), 1481-1486. doi:10.1126/science. 1252254

dos Santos, R. G., Carlos Bouso, J., Angel Alcazar-Corcoles, M., \& Hallak, J. E. C. (2018). Efficacy, tolerability, and safety of serotonergic psychedelics for the management of mood, anxiety, and substance-use disorders: a systematic review of systematic reviews. Expert Review of Clinical Pharmacology, 11(9), 889-902. doi:10.1080/17512433.2018.1511424

Dos Santos, R. G., \& Hallak, J. E. C. (2020). Therapeutic use of serotoninergic hallucinogens: A review of the evidence and of the biological and psychological mechanisms. Neurosci Biobehav Rev, 108, 423-434. doi:10.1016/j.neubiorev.2019.12.001

Dworetsky, A., Seitzman, B. A., Adeyemo, B., Neta, M., Coalson, R. S., Petersen, S. E., \& Gratton, C. (2021). Probabilistic mapping of human functional brain networks identifies regions of high group consensus. NeuroImage, 118164. doi:https://doi.org/10.1016/j.neuroimage.2021.118164

Dyck, E. (2005). Flashback: psychiatric experimentation with LSD in historical perspective. Can J Psychiatry, 50(7), 381-388. doi:10.1177/070674370505000703

Epstein, R. A. (2008). Parahippocampal and retrosplenial contributions to human spatial navigation. Trends in Cognitive Sciences, 12(10), 388-396. doi:10.1016/j.tics.2008.07.004

Etkin, A., Egner, T., \& Kalisch, R. (2011). Emotional processing in anterior cingulate and medial prefrontal cortex. Trends in Cognitive Sciences, 15(2), 85-93. doi:10.1016/j.tics.2010.11.004

Etkin, A., Egner, T., Peraza, D. M., Kandel, E. R., \& Hirsch, J. (2006). Resolving emotional conflict: a role for the rostral anterior cingulate cortex in modulating activity in the amygdala. Neuron, 51(6), 871-882. doi:10.1016/j.neuron.2006.07.029

Euston, D. R., Gruber, A. J., \& McNaughton, B. L. (2012). The role of medial prefrontal cortex in memory and decision making. Neuron, 76(6), 1057-1070. doi:10.1016/j.neuron.2012.12.002

Fahrenfort, J. J., Snijders, T. M., Heinen, K., van Gaal, S., Scholte, H. S., \& Lamme, V. A. F. (2012). Neuronal integration in visual cortex elevates face category tuning to conscious face perception. Proceedings of the National Academy of Sciences, 109(52), 21504-21509. doi:10.1073/pnas.1207414110

Fan, J. (2014). An information theory account of cognitive control. Frontiers in Human Neuroscience, 8(680). doi:10.3389/fnhum.2014.00680

Fan, J., Van Dam, N. T., Gu, X., Liu, X., Wang, H., Tang, C. Y., \& Hof, P. R. (2014). Quantitative characterization of functional anatomical contributions to cognitive control under uncertainty. J Cogn Neurosci, 26(7), 1490-1506. doi:10.1162/jocn_a_00554

Farrer, C., \& Frith, C. D. (2002). Experiencing oneself vs another person as being the cause of an action: the neural correlates of the experience of agency. NeuroImage, 15(3), 596-603. doi:10.1006/nimg.2001.1009 
Feldman, H., \& Friston, K. J. (2010). Attention, uncertainty, and free-energy. Frontiers in Human Neuroscience, 4, 215.

Felleman, D. J., \& Van Essen, D. C. (1991). Distributed hierarchical processing in the primate cerebral cortex. Cereb Cortex, 1(1), 1-47. doi:10.1093/cercor/1.1.1

Festinger, L. (1962). Cognitive dissonance. Sci Am, 207, 93-102. doi:10.1038/scientificamerican1062-93

Fogwe LA, R. V., Mesfin FB. (2021). Neuroanatomy, Hippocampus. Treasure Island (FL): StatPearls Publishing.

Fotopoulou, A., \& Tsakiris, M. (2017). Mentalizing homeostasis: The social origins of interoceptive inference. Neuropsychoanalysis, 19(1), 3-28. doi:10.1080/15294145.2017.1294031

Fox, M. D., \& Raichle, M. E. (2007). Spontaneous fluctuations in brain activity observed with functional magnetic resonance imaging. Nature Reviews Neuroscience, 8(9), 700-711. doi:10.1038/nrn2201

Fox, M. D., Snyder, A. Z., Vincent, J. L., Corbetta, M., Van Essen, D. C., \& Raichle, M. E. (2005). The human brain is intrinsically organized into dynamic, anticorrelated functional networks. Proceedings of the National Academy of Sciences of the United States of America, 102(27), 9673. doi:10.1073/pnas.0504136102

Friston, K. (2008). Hierarchical Models in the Brain. PLOS Computational Biology, 4(11), e1000211. doi:10.1371/journal.pcbi.1000211

Friston, K. (2009). The free-energy principle: a rough guide to the brain? Trends in Cognitive Sciences, 13(7), 293-301. doi:https://doi.org/10.1016/j.tics.2009.04.005

Friston, K. (2010). The free-energy principle: a unified brain theory? Nature Reviews Neuroscience, 11, 127. doi:10.1038/nrn2787

https://www.nature.com/articles/nrn2787\#supplementary-information

Friston, K. (2018). Does predictive coding have a future? Nature Neuroscience, 21(8), 10191021. doi:10.1038/s41593-018-0200-7

Friston, K. J. (2011). Functional and effective connectivity: a review. Brain Connect, 1(1), 13-36. doi:10.1089/brain.2011.0008

Friston, K. J. (2020). Bayesian Dysconnections. American Journal of Psychiatry, 177(12), 1110-1112. doi:10.1176/appi.ajp.2020.20091421

Friston, K. J., Kahan, J., Biswal, B., \& Razi, A. (2014). A DCM for resting state fMRI. Neuroimage, 94, 396-407. doi:10.1016/j.neuroimage.2013.12.009

Fulcher, B. D., \& Fornito, A. (2016). A transcriptional signature of hub connectivity in the mouse connectome. Proceedings of the National Academy of Sciences, 113(5), 14351440. doi:10.1073/pnas. 1513302113

Fusar-Poli, P., Allen, P., Bhattacharyya, S., Crippa, J. A., Mechelli, A., Borgwardt, S., . . McGuire, P. (2010). Modulation of effective connectivity during emotional processing by Delta 9-tetrahydrocannabinol and cannabidiol. Int $J$ Neuropsychopharmacol, 13(4), 421-432. doi:10.1017/s1461145709990617

Garcia-Romeu, A., Barrett, F. S., Carbonaro, T. M., Johnson, M. W., \& Griffiths, R. R. (2021). Optimal dosing for psilocybin pharmacotherapy: Considering weight-adjusted and fixed dosing approaches. Journal of Psychopharmacology, 35(4), 353-361. doi:10.1177/0269881121991822

Garcia-Romeu, A., Griffiths, R. R., \& Johnson, M. W. (2014). Psilocybin-occasioned mystical experiences in the treatment of tobacco addiction. Current drug abuse reviews, 7(3), 157-164. doi:10.2174/1874473708666150107121331

Geyer, M. A., \& Vollenweider, F. X. (2008). Serotonin research: contributions to understanding psychoses. Trends in Pharmacological Sciences, 29(9), 445-453. doi:https://doi.org/10.1016/j.tips.2008.06.006 
Ghashghaei, H. T., Hilgetag, C. C., \& Barbas, H. (2007). Sequence of information processing for emotions based on the anatomic dialogue between prefrontal cortex and amygdala. NeuroImage, 34(3), 905-923. doi:10.1016/j.neuroimage.2006.09.046

Goldstein-Piekarski, A. N., Staveland, B. R., Ball, T. M., Yesavage, J., Korgaonkar, M. S., \& Williams, L. M. (2018). Intrinsic functional connectivity predicts remission on antidepressants: a randomized controlled trial to identify clinically applicable imaging biomarkers. Translational psychiatry, 8(1), 57. doi:10.1038/s41398-018-0100-3

Gotink, R. A., Vernooij, M. W., Ikram, M. A., Niessen, W. J., Krestin, G. P., Hofman, A., . . . Hunink, M. G. M. (2018). Meditation and yoga practice are associated with smaller right amygdala volume: the Rotterdam study. Brain imaging and behavior, 12(6), 1631-1639. doi:10.1007/s11682-018-9826-z

Greicius, M. D., \& Menon, V. (2004). Default-Mode Activity during a Passive Sensory Task: Uncoupled from Deactivation but Impacting Activation. J Cogn Neurosci, 16(9), 1484-1492. doi:10.1162/0898929042568532

Griffa, A., Baumann, P. S., Klauser, P., Mullier, E., Cleusix, M., Jenni, R., . . Hagmann, P. (2019). Brain connectivity alterations in early psychosis: from clinical to neuroimaging staging. Translational psychiatry, 9(1), 62. doi:10.1038/s41398-0190392-y

Griffiths, R. R., Johnson, M. W., Carducci, M. A., Umbricht, A., Richards, W. A., Richards, B. D., ... Klinedinst, M. A. (2016). Psilocybin produces substantial and sustained decreases in depression and anxiety in patients with life-threatening cancer: A randomized double-blind trial. Journal of Psychopharmacology, 30(12), 1181-1197. doi:10.1177/0269881116675513

Griffiths, R. R., Johnson, M. W., Richards, W. A., Richards, B. D., Jesse, R., MacLean, K. A., ... Klinedinst, M. A. (2018). Psilocybin-occasioned mystical-type experience in combination with meditation and other spiritual practices produces enduring positive changes in psychological functioning and in trait measures of prosocial attitudes and behaviors. Journal of Psychopharmacology, 32(1), 49-69. doi:10.1177/0269881117731279

Griffiths, R. R., Johnson, M. W., Richards, W. A., Richards, B. D., McCann, U., \& Jesse, R. (2011). Psilocybin occasioned mystical-type experiences: immediate and persisting dose-related effects. Psychopharmacology, 218(4), 649-665. doi:10.1007/s00213011-2358-5

Griffiths, R. R., Richards, W. A., McCann, U., \& Jesse, R. (2006). Psilocybin can occasion mystical-type experiences having substantial and sustained personal meaning and spiritual significance. Psychopharmacology, 187(3), 268-283. doi:10.1007/s00213006-0457-5

Grof, S. (1980). LSD Psychotherapy. Alameda, CA: Hunter House Publishers.

Gusnard, D. A., Akbudak, E., Shulman, G. L., \& Raichle, M. E. (2001). Medial Prefrontal Cortex and Self-Referential Mental Activity: Relation to a Default Mode of Brain Function. Proceedings of the National Academy of Sciences of the United States of America, 98(7), 4259-4264. Retrieved from http://www.jstor.org.ezproxy.lib.monash.edu.au/stable/3055404

https://www.ncbi.nlm.nih.gov/pmc/articles/PMC31213/pdf/pq004259.pdf

Hagmann, P., Cammoun, L., Gigandet, X., Meuli, R., Honey, C. J., Wedeen, V. J., \& Sporns, O. (2008). Mapping the structural core of human cerebral cortex. PLoS biology, 6(7), e159-e159. doi:10.1371/journal.pbio.0060159

Halberstadt, A. L. (2015). Recent advances in the neuropsychopharmacology of serotonergic hallucinogens. Behav Brain Res, 277, 99-120. doi:10.1016/j.bbr.2014.07.016 
Halgren, E., Walter, R. D., Cherlow, D. G., \& Crandall, P. H. (1978). Mental phenomena evoked by electrical stimulation of the human hippocampal formation and amygdala. Brain, 101(1), 83-117. doi:10.1093/brain/101.1.83

Ham, T. E., Bonnelle, V., Hellyer, P., Jilka, S., Robertson, I. H., Leech, R., \& Sharp, D. J. (2014). The neural basis of impaired self-awareness after traumatic brain injury. Brain : a journal of neurology, 137(Pt 2), 586-597. doi:10.1093/brain/awt350

Hariri, A. R., Bookheimer, S. Y., \& Mazziotta, J. C. (2000). Modulating emotional responses: effects of a neocortical network on the limbic system. Neuroreport, 11(1), 43-48. doi:10.1097/00001756-200001170-00009

Hartogsohn, I. (2018). The Meaning-Enhancing Properties of Psychedelics and Their Mediator Role in Psychedelic Therapy, Spirituality, and Creativity. Frontiers in Neuroscience, 12. doi:10.3389/fnins.2018.00129

Hasenkamp, W., Wilson-Mendenhall, C. D., Duncan, E., \& Barsalou, L. W. (2012). Mind wandering and attention during focused meditation: a fine-grained temporal analysis of fluctuating cognitive states. NeuroImage, 59(1), 750-760. doi:10.1016/j.neuroimage.2011.07.008

Heilbronner, S. R., \& Hayden, B. Y. (2016). Dorsal Anterior Cingulate Cortex: A Bottom-Up View. Annu Rev Neurosci, 39, 149-170. doi:10.1146/annurev-neuro-070815-013952

Hermle, L., Fünfgeld, M., Oepen, G., Botsch, H., Borchardt, D., Gouzoulis, E., . . . Spitzer, M. (1992). Mescaline-induced psychopathological, neuropsychological, and neurometabolic effects in normal subjects: experimental psychosis as a tool for psychiatric research. Biol Psychiatry, 32(11), 976-991. doi:10.1016/00063223(92)90059-9

Herzog, R., Mediano, P. A. M., Rosas, F. E., Carhart-Harris, R., Perl, Y. S., Tagliazucchi, E., $\&$ Cofre, R. (2020). A mechanistic model of the neural entropy increase elicited by psychedelic drugs. Scientific Reports, 10(1), 17725. doi:10.1038/s41598-020-74060-6

Heuschkel, K., \& Kuypers, K. P. C. (2020). Depression, Mindfulness, and Psilocybin: Possible Complementary Effects of Mindfulness Meditation and Psilocybin in the Treatment of Depression. A Review. Frontiers in Psychiatry, 11, 224-224. doi:10.3389/fpsyt.2020.00224

Hipp, J. F., Engel, A. K., \& Siegel, M. (2011). Oscillatory synchronization in large-scale cortical networks predicts perception. Neuron, 69(2), 387-396. doi:10.1016/j.neuron.2010.12.027

Hirschfeld, T., \& Schmidt, T. T. (2020). How does it feel to be on psilocybin? Dose-response relationships of subjective experiences in humans. bioRxiv, 2020.2006.2009.142802. doi:10.1101/2020.06.09.142802

Hobson, J. A., Gott, J. A., \& Friston, K. J. (2021). Minds and Brains, Sleep and Psychiatry. Psychiatric Research and Clinical Practice, 3(1), 12-28. doi:https://doi.org/10.1176/appi.prcp.20200023

Hohwy, J. (2016). The Self-Evidencing Brain. Noûs, 50(2), 259-285. doi:10.1111/nous.12062

Holmes, J., \& Nolte, T. (2019). "Surprise" and the Bayesian Brain: Implications for Psychotherapy Theory and Practice. Frontiers in Psychology, 10(592). doi:10.3389/fpsyg.2019.00592

Hopper, J. W., Frewen, P. A., van der Kolk, B. A., \& Lanius, R. A. (2007). Neural correlates of reexperiencing, avoidance, and dissociation in PTSD: symptom dimensions and emotion dysregulation in responses to script-driven trauma imagery. J Trauma Stress, 20(5), 713-725. doi:10.1002/jts.20284

Houben, M., Claes, L., Sleuwaegen, E., Berens, A., \& Vansteelandt, K. (2018). Emotional reactivity to appraisals in patients with a borderline personality disorder: a daily life 
study. Borderline Personality Disorder and Emotion Dysregulation, 5(1), 18. doi:10.1186/s40479-018-0095-7

Huettel, S. A., Song, A. W., \& McCarthy, G. (2005). Decisions under uncertainty: probabilistic context influences activation of prefrontal and parietal cortices. The Journal of neuroscience : the official journal of the Society for Neuroscience, 25(13), 3304-3311. doi:10.1523/JNEUROSCI.5070-04.2005

Ide, J. S., Shenoy, P., Yu, A. J., \& Li, C. S. (2013). Bayesian prediction and evaluation in the anterior cingulate cortex. The Journal of Neuroscience, 33(5), 2039-2047. doi:10.1523/jneurosci.2201-12.2013

Illman, N. A., Butler, C. R., Souchay, C., \& Moulin, C. J. A. (2012). Déjà experiences in temporal lobe epilepsy. Epilepsy research and treatment, 2012, 539567-539567. doi:10.1155/2012/539567

Inserra, A., De Gregorio, D., \& Gobbi, G. (2021). Psychedelics in Psychiatry: Neuroplastic, Immunomodulatory, and Neurotransmitter Mechanisms. Pharmacological Reviews, 73(1), 202. doi:10.1124/pharmrev.120.000056

Johnson, M., Richards, W., \& Griffiths, R. (2008). Human hallucinogen research: guidelines for safety. In (Vol. 22, pp. 603-620). London, England.

Johnson, M. E. (2019). Neural Annealing: Toward a Neural Theory of Everything. Retrieved from https://opentheory.net/2019/11/neural-annealing-toward-a-neural-theory-ofeverything/

Johnson, M. W., Garcia-Romeu, A., Cosimano, M. P., \& Griffiths, R. R. (2014). Pilot study of the 5-HT2AR agonist psilocybin in the treatment of tobacco addiction. Journal of psychopharmacology (Oxford, England), 28(11), 983-992. doi:10.1177/0269881114548296

Johnson, M. W., Griffiths, R. R., Hendricks, P. S., \& Henningfield, J. E. (2018). The abuse potential of medical psilocybin according to the 8 factors of the Controlled Substances Act. Psychedelics: New Doors, Altered Perceptions, 142, 143-166. doi:10.1016/j.neuropharm.2018.05.012

Josipovic, Z., Dinstein, I., Weber, J., \& Heeger, D. (2012). Influence of meditation on anticorrelated networks in the brain. Frontiers in Human Neuroscience, 5, 183. Retrieved from https://www.frontiersin.org/article/10.3389/fnhum.2011.00183

https://www.ncbi.nlm.nih.gov/pmc/articles/PMC3250078/pdf/fnhum-05-00183.pdf

Kaelen, M., Giribaldi, B., Raine, J., Evans, L., Timmerman, C., Rodriguez, N., . . CarhartHarris, R. (2018). The hidden therapist: evidence for a central role of music in psychedelic therapy. Psychopharmacology, 235(2), 505-519. doi:10.1007/s00213017-4820-5

Kaelen, M., Roseman, L., Kahan, J., Santos-Ribeiro, A., Orban, C., Lorenz, R., . . CarhartHarris, R. (2016). LSD modulates music-induced imagery via changes in parahippocampal connectivity. European Neuropsychopharmacology, 26(7), 10991109. doi:https://doi.org/10.1016/j.euroneuro.2016.03.018

Kaiser, R. H., Andrews-Hanna, J. R., Wager, T. D., \& Pizzagalli, D. A. (2015). Large-Scale Network Dysfunction in Major Depressive Disorder: A Meta-analysis of RestingState Functional Connectivity. JAMA Psychiatry, 72(6), 603-611. doi:10.1001/jamapsychiatry.2015.0071

Kalat, J. W. (2014). Consciousness and the Brain: Deciphering How the Brain Codes our Thoughts. Journal of Undergraduate Neuroscience Education, 12(2), R5-R6. Retrieved from https://www.ncbi.nlm.nih.gov/pmc/articles/PMC3971003/

Kam, J. W. Y., Lin, J. J., Solbakk, A.-K., Endestad, T., Larsson, P. G., \& Knight, R. T. (2019). Default network and frontoparietal control network theta connectivity 
supports internal attention. Nature Human Behaviour. doi:10.1038/s41562-019-07170

Kanai, R., Komura, Y., Shipp, S., \& Friston, K. (2015). Cerebral hierarchies: predictive processing, precision and the pulvinar. Philos Trans $R$ Soc Lond B Biol Sci, 370(1668). doi:10.1098/rstb.2014.0169

Kanai, R., Komura, Y., Shipp, S., \& Friston, K. (2015). Cerebral hierarchies: predictive processing, precision and the pulvinar. Philosophical Transactions of the Royal Society B: Biological Sciences, 370(1668), 20140169. doi:10.1098/rstb.2014.0169

Kaplan, J., Gimbel, S., \& Harris, S. (2016). Neural correlates of maintaining one's political beliefs in the face of counterevidence. Scientific Reports, 6, 39589. doi:10.1038/srep39589

Keng, S.-L., Smoski, M. J., \& Robins, C. J. (2011). Effects of mindfulness on psychological health: a review of empirical studies. Clinical psychology review, 31(6), 1041-1056. doi:10.1016/j.cpr.2011.04.006

Kiebel, S. J., Daunizeau, J., \& Friston, K. J. (2008). A Hierarchy of Time-Scales and the Brain. PLOS Computational Biology, 4(11), e1000209. doi:10.1371/journal.pcbi.1000209

Kim, M., \& Maguire, E. A. (2018). Hippocampus, retrosplenial and parahippocampal cortices encode multi-compartment 3D space in a hierarchical manner. bioRxiv, 200576. doi:10.1101/200576

Klaassens, B. L., van Gorsel, H. C., Khalili-Mahani, N., van der Grond, J., Wyman, B. T., Whitcher, B., . . . van Gerven, J. M. (2015). Single-dose serotonergic stimulation shows widespread effects on functional brain connectivity. NeuroImage, 122, 440450. doi:10.1016/j.neuroimage.2015.08.012

Kometer, M., Pokorny, T., Seifritz, E., \& Volleinweider, F. X. (2015). Psilocybin-induced spiritual experiences and insightfulness are associated with synchronization of neuronal oscillations. Psychopharmacology, 232(19), 3663-3676. doi:10.1007/s00213-015-4026-7

Komorowski, A., James, G. M., Philippe, C., Gryglewski, G., Bauer, A., Hienert, M., . . . Lanzenberger, R. (2017). Association of Protein Distribution and Gene Expression Revealed by PET and Post-Mortem Quantification in the Serotonergic System of the Human Brain. Cereb Cortex, 27(1), 117-130. doi:10.1093/cercor/bhw355

Kraehenmann, R., Preller, K. H., Scheidegger, M., Pokorny, T., Bosch, O. G., Seifritz, E., \& Vollenweider, F. X. (2015). Psilocybin-Induced Decrease in Amygdala Reactivity Correlates with Enhanced Positive Mood in Healthy Volunteers. Biological Psychiatry, 78(8), 572-581. doi:10.1016/j.biopsych.2014.04.010

Kraehenmann, R., Schmidt, A., Friston, K., Preller, K. H., Seifritz, E., \& Vollenweider, F. X. (2016). The mixed serotonin receptor agonist psilocybin reduces threat-induced modulation of amygdala connectivity. Neuroimage-Clinical, 11, 53-60. doi:10.1016/j.nicl.2015.08.009

Krimmel, S. R., White, M. G., Panicker, M. H., Barrett, F. S., Mathur, B. N., \& Seminowicz, D. A. (2019). Resting state functional connectivity and cognitive task-related activation of the human claustrum. NeuroImage, 196, 59-67. doi:10.1016/j.neuroimage.2019.03.075

LaFarge, L. (2011). Defense and Resistance. In Textbook of Psychoanalysis (2 ed.). Laumann, T. O., Snyder, A. Z., Mitra, A., Gordon, E. M., Gratton, C., Adeyemo, B., . . . Petersen, S. E. (2017). On the Stability of BOLD fMRI Correlations. Cerebral Cortex, 27(10), 4719-4732. doi:10.1093/cercor/bhw265

Lebedev, A. V., Kaelen, M., Lövdén, M., Nilsson, J., Feilding, A., Nutt, D. J., \& CarhartHarris, R. L. (2016). LSD-induced entropic brain activity predicts subsequent 
personality change. Human Brain Mapping, 37(9), 3203-3213.

doi:10.1002/hbm.23234

Lebedev, A. V., Lövdén, M., Rosenthal, G., Feilding, A., Nutt, D. J., \& Carhart-Harris, R. L. (2015). Finding the self by losing the self: Neural correlates of ego-dissolution under psilocybin. Human Brain Mapping, 36(8), 3137-3153. doi:10.1002/hbm.22833

Leech, R., \& Sharp, D. J. (2014). The role of the posterior cingulate cortex in cognition and disease. Brain : a journal of neurology, 137(Pt 1), 12-32. doi:10.1093/brain/awt162

Legrand, D., \& Ruby, P. (2009). What is self-specific? Theoretical investigation and critical review of neuroimaging results [American Psychological Association doi:10.1037/a0014172]. Retrieved

Lemche, E., Surguladze, S. A., Brammer, M. J., Phillips, M. L., Sierra, M., David, A. S., . . Giampietro, V. P. (2016). Dissociable brain correlates for depression, anxiety, dissociation, and somatization in depersonalization-derealization disorder. CNS Spectr, 21(1), 35-42. doi:10.1017/s1092852913000588

Leptourgos, P., Fortier-Davy, M., Carhart-Harris, R., Corlett, P., Dupuis, D., Halberstadt, A., ... Jardri, R. (2020). Hallucinations Under Psychedelics and in the Schizophrenia Spectrum: An Interdisciplinary and Multiscale Comparison. Schizophrenia Bulletin. doi:10.1093/schbul/sbaa117

Letheby, C. (2016). The epistemic innocence of psychedelic states. Consciousness and Cognition, 39, 28-37. doi:10.1016/j.concog.2015.11.012

Letheby, C., \& Gerrans, P. (2017a). Self unbound: ego dissolution in psychedelic experience. Neuroscience of Consciousness, 2017(1). doi:10.1093/nc/nix016

Letheby, C., \& Gerrans, P. (2017b). Self unbound: ego dissolution in psychedelic experience. Open Forum Infectious Diseases, 4(1). doi:10.1093/nc/nix016

Lewis, C. R., Preller, K. H., Braden, B. B., Riecken, C., \& Vollenweider, F. X. (2020). Rostral Anterior Cingulate Thickness Predicts the Emotional Psilocybin Experience. Biomedicines, 8(2). doi:10.3390/biomedicines 8020034

Li, S., Hu, N., Zhang, W., Tao, B., Dai, J., Gong, Y., . . Lui, S. (2019). Dysconnectivity of Multiple Brain Networks in Schizophrenia: A Meta-Analysis of Resting-State Functional Connectivity. Frontiers in Psychiatry, 10(482). doi:10.3389/fpsyt.2019.00482

Liang, X., He, Y., Salmeron, B. J., Gu, H., Stein, E. A., \& Yang, Y. (2015). Interactions between the salience and default-mode networks are disrupted in cocaine addiction. The Journal of neuroscience : the official journal of the Society for Neuroscience, 35(21), 8081-8090. doi:10.1523/JNEUROSCI.3188-14.2015

Liechti, M. E. (2017). Modern Clinical Research on LSD. Neuropsychopharmacology, 42(11), 2114-2127. doi:10.1038/npp.2017.86

Limanowski, J., \& Blankenburg, F. (2013). Minimal self-models and the free energy principle. Frontiers in Human Neuroscience, 7, 547-547. doi:10.3389/fnhum.2013.00547

Liotti, M., Mayberg, H. S., Brannan, S. K., McGinnis, S., Jerabek, P., \& Fox, P. T. (2000). Differential limbic\&\#x2013; cortical correlates of sadness and anxiety in healthy subjects: implications for affective disorders. Biological Psychiatry, 48(1), 30-42. doi:10.1016/S0006-3223(00)00874-X

Lord, L.-D., Expert, P., Atasoy, S., Roseman, L., Rapuano, K., Lambiotte, R., . . Cabral, J. (2018). Altered trajectories in the dynamical repertoire of functional network states under psilocybin. bioRxiv, 376491. doi:10.1101/376491

Lord, L.-D., Expert, P., Atasoy, S., Roseman, L., Rapuano, K., Lambiotte, R., . . Cabral, J. (2019). Dynamical exploration of the repertoire of brain networks at rest is modulated 
by psilocybin. NeuroImage, 199, 127-142.

doi:https://doi.org/10.1016/j.neuroimage.2019.05.060

Luppi, A. I., Carhart-Harris, R. L., Roseman, L., Pappas, I., Menon, D. K., \& Stamatakis, E. A. (2021). LSD alters dynamic integration and segregation in the human brain. NeuroImage, 227, 117653. doi:https://doi.org/10.1016/j.neuroimage.2020.117653

Ly, C., Greb, A. C., Cameron, L. P., Wong, J. M., Barragan, E. V., Wilson, P. C., . . Olson, D. E. (2018). Psychedelics Promote Structural and Functional Neural Plasticity. Cell reports, 23(11), 3170-3182. doi:10.1016/j.celrep.2018.05.022

Maclean, K. A., Leoutsakos, J.-M. S., Johnson, M. W., \& Griffiths, R. R. (2012). Factor Analysis of the Mystical Experience Questionnaire: A Study of Experiences Occasioned by the Hallucinogen Psilocybin. Journal for the Scientific Study of Religion, 51(4), 721-737. doi:10.1111/j.1468-5906.2012.01685.x

Madsen, M. K., Fisher, P. M., Burmester, D., Dyssegaard, A., Stenbæk, D. S., Kristiansen, S., ... Knudsen, G. M. (2019). Psychedelic effects of psilocybin correlate with serotonin 2A receptor occupancy and plasma psilocin levels. Neuropsychopharmacology, 44(7), 1328. doi:10.1038/s41386-019-0324-9

Majic, T., Schmidt, T. T., \& Gallinat, J. (2015). Peak experiences and the afterglow phenomenon: when and how do therapeutic effects of hallucinogens depend on psychedelic experiences? J Psychopharmacol, 29(3), 241-253. doi:10.1177/0269881114568040

Malone, T. C., Mennenga, S. E., Guss, J., Podrebarac, S. K., Owens, L. T., Bossis, A. P., . . . Ross, S. (2018). Individual Experiences in Four Cancer Patients Following Psilocybin-Assisted Psychotherapy. Frontiers in Pharmacology, 9. doi:10.3389/fphar.2018.00256

Marek, S., \& Dosenbach, N. U. F. (2018). The frontoparietal network: function, electrophysiology, and importance of individual precision mapping. Dialogues in clinical neuroscience, 20(2), 133-140. Retrieved from https://www.ncbi.nlm.nih.gov/pubmed/30250390

https://www.ncbi.nlm.nih.gov/pmc/articles/PMC6136121/

https://www.ncbi.nlm.nih.gov/pmc/articles/PMC6136121/pdf/DialoguesClinNeurosci-20133.pdf

Marshall, P. (2005). Mind Beyond the Brain: Reducing Valves and Metaphysics. doi:10.1093/0199279438.003.0009

Matias, S., Lottem, E., Dugué, G. P., \& Mainen, Z. F. (2017). Activity patterns of serotonin neurons underlying cognitive flexibility. eLife, 6, e20552. doi:10.7554/eLife.20552

Mediano, P. A. M., Rosas, F. E., Timmermann, C., Roseman, L., Nutt, D. J., Feilding, A., . . . Carhart-Harris, R. L. (2020). Effects of external stimulation on psychedelic state neurodynamics. bioRxiv, 2020.2011.2001.356071. doi:10.1101/2020.11.01.356071

Melloni, L., Molina, C., Pena, M., Torres, D., Singer, W., \& Rodriguez, E. (2007). Synchronization of neural activity across cortical areas correlates with conscious perception. The Journal of Neuroscience, 27(11), 2858-2865. doi:10.1523/jneurosci.4623-06.2007

Menon, V. (2011). Large-scale brain networks and psychopathology: a unifying triple network model. Trends Cogn Sci, 15(10), 483-506. doi:10.1016/j.tics.2011.08.003

Menon, V. (2015). Salience Network (Vol. 2): Elsevier.

Menon, V. (2018). The Triple Network Model, Insight, and Large-Scale Brain Organization in Autism. Biological Psychiatry, 84(4), 236-238.

doi:10.1016/j.biopsych.2018.06.012 
Menon, V., \& Uddin, L. Q. (2010). Saliency, switching, attention and control: a network model of insula function. Brain structure \& function, 214(5-6), 655-667.

doi:10.1007/s00429-010-0262-0

Mertens, L. J., Wall, M. B., Roseman, L., Demetriou, L., Nutt, D. J., \& Carhart-Harris, R. L. (2020). Therapeutic mechanisms of psilocybin: Changes in amygdala and prefrontal functional connectivity during emotional processing after psilocybin for treatmentresistant depression. J Psychopharmacol, 34(2), 167-180. doi:10.1177/0269881119895520

Metzinger, T. (2003). Being no one: The self-model theory of subjectivity. Cambridge, MA, US: MIT Press.

Milad, M. R., \& Quirk, G. J. (2002). Neurons in medial prefrontal cortex signal memory for fear extinction. Nature, 420(6911), 70-74. doi:10.1038/nature01138

Miller, L., Balodis, I. M., McClintock, C. H., Xu, J., Lacadie, C. M., Sinha, R., \& Potenza, M. N. (2019). Neural Correlates of Personalized Spiritual Experiences. Cerebral Cortex, 29(6), 2331-2338. doi:10.1093/cercor/bhy 102

Millière, R. (2017). Looking for the Self: Phenomenology, Neurophysiology and Philosophical Significance of Drug-induced Ego Dissolution. Frontiers in Human Neuroscience, 11(245). doi:10.3389/fnhum.2017.00245

Millière, R., Carhart-Harris, R. L., Roseman, L., Trautwein, F.-M., \& Berkovich-Ohana, A. (2018). Psychedelics, Meditation, and Self-Consciousness. Frontiers in Psychology, 9(1475). doi:10.3389/fpsyg.2018.01475

Mohr, C., \& Claridge, G. (2015). Schizotypy--do not worry, it is not all worrisome. Schizophr Bull, 41 Suppl 2, S436-443. doi:10.1093/schbul/sbu185

Mueller, F., Lenz, C., Dolder, P. C., Harder, S., Schmid, Y., Lang, U. E., . . Borgwardt, S. (2017). Acute effects of LSD on amygdala activity during processing of fearful stimuli in healthy subjects. Translational psychiatry, 7(4), e1084-e1084. doi:10.1038/tp.2017.54

Müller, F., Dolder, P. C., Schmidt, A., Liechti, M. E., \& Borgwardt, S. (2018). Altered network hub connectivity after acute LSD administration. NeuroImage. Clinical, 18, 694-701. doi:10.1016/j.nicl.2018.03.005

Müller, F., Lenz, C., Dolder, P., Lang, U., Schmidt, A., Liechti, M., \& Borgwardt, S. (2017). Increased thalamic resting-state connectivity as a core driver of LSD-induced hallucinations. Acta Psychiatrica Scandinavica. Retrieved from https://onlinelibrary.wiley.com/doi/abs/10.1111/acps.12818

files/132/acps.html

Mumford, D. (1992). On the computational architecture of the neocortex. Biological Cybernetics, 66(3), 241-251. doi:10.1007/BF00198477

Murphy, K., Birn, R. M., Handwerker, D. A., Jones, T. B., \& Bandettini, P. A. (2009). The impact of global signal regression on resting state correlations: are anti-correlated networks introduced? NeuroImage, 44(3), 893-905. doi:10.1016/j.neuroimage.2008.09.036

Muthukumaraswamy, S. D., Carhart-Harris, R. L., Moran, R. J., Brookes, M. J., Williams, T. M., Errtizoe, D., . . . Nutt, D. J. (2013). Broadband Cortical Desynchronization Underlies the Human Psychedelic State. The Journal of Neuroscience, 33(38), 1517115183. doi:10.1523/jneurosci.2063-13.2013

Muthukumaraswamy, S. D., \& Liley, D. T. J. (2018). 1/f electrophysiological spectra in resting and drug-induced states can be explained by the dynamics of multiple oscillatory relaxation processes. NeuroImage, 179, 582-595.

doi:10.1016/j.neuroimage.2018.06.068 
Nichols, D. E. (2016). Psychedelics. Pharmacological Reviews, 68(2), 264-355. doi:10.1124/pr.115.011478

Northoff, G., Heinzel, A., de Greck, M., Bermpohl, F., Dobrowolny, H., \& Panksepp, J. (2006). Self-referential processing in our brain--a meta-analysis of imaging studies on the self. NeuroImage, 31(1), 440-457. doi:10.1016/j.neuroimage.2005.12.002

Nour, M. M., Evans, L., Nutt, D., \& Carhart-Harris, R. L. (2016). Ego-Dissolution and Psychedelics: Validation of the Ego-Dissolution Inventory (EDI). Frontiers in Human Neuroscience, 10. doi:10.3389/fnhum.2016.00269

Nutt, D., \& Carhart-Harris, R. (2020). The Current Status of Psychedelics in Psychiatry. JAMA Psychiatry. doi:10.1001/jamapsychiatry.2020.2171

O'Driscoll, K., \& Leach, J. P. (1998). "No longer Gage": an iron bar through the head. Early observations of personality change after injury to the prefrontal cortex. BMJ (Clinical research ed.), 317(7174), 1673-1674. doi:10.1136/bmj.317.7174.1673a

Ochsner, K. N., \& Gross, J. J. (2005). The cognitive control of emotion. Trends Cogn Sci, 9(5), 242-249. doi:10.1016/j.tics.2005.03.010

Oldham, S., Arnatkeviciūtè, A., Smith, R. E., Tiego, J., Bellgrove, M. A., \& Fornito, A. (2020). The efficacy of different preprocessing steps in reducing motion-related confounds in diffusion MRI connectomics. bioRxiv, 2020.2003.2025.008979. doi:10.1101/2020.03.25.008979

Olson, D. E. (2020). The Subjective Effects of Psychedelics May Not Be Necessary for Their Enduring Therapeutic Effects. ACS Pharmacology \& Translational Science. doi:10.1021/acsptsci.0c00192

Osmond, H. (1957). A review of the clinical effects of psychotomimetic agents. Ann NY Acad Sci, 66(3), 418-434. doi:10.1111/j.1749-6632.1957.tb40738.x

Palhano-Fontes, F., Andrade, K. C., Tofoli, L. F., Santos, A. C., Crippa, J. A., Hallak, J. E., . . . de Araujo, D. B. (2015). The psychedelic state induced by ayahuasca modulates the activity and connectivity of the default mode network. Plos One, 10(2), e0118143. doi:10.1371/journal.pone.0118143

Palva, J. M., Palva, S., \& Kaila, K. (2005). Phase Synchrony among Neuronal Oscillations in the Human Cortex. The Journal of Neuroscience, 25(15), 3962-3972. doi:10.1523/jneurosci.4250-04.2005

Parkes, L., Fulcher, B., Yücel, M., \& Fornito, A. (2018). An evaluation of the efficacy, reliability, and sensitivity of motion correction strategies for resting-state functional MRI. NeuroImage, 171, 415-436. doi:https://doi.org/10.1016/j.neuroimage.2017.12.073

Payne, J. E., Chambers, R., \& Liknaitzky, P. (2021). Combining Psychedelic and Mindfulness Interventions: Synergies to Inform Clinical Practice. ACS Pharmacology \& Translational Science, 4(2), 416-423. doi:10.1021/acsptsci.1c00034

Pazos, A., Probst, A., \& Palacios, J. M. (1987). Serotonin receptors in the human brain--IV. Autoradiographic mapping of serotonin-2 receptors. Neuroscience, 21(1), 123-139. doi:10.1016/0306-4522(87)90327-7

Petri, G., Expert, P., Turkheimer, F., Carhart-Harris, R., Nutt, D., Hellyer, P. J., \& Vaccarino, F. (2014). Homological scaffolds of brain functional networks. Journal of The Royal Society Interface, 11(101), 20140873. doi:10.1098/rsif.2014.0873

Phelps, E. A., \& LeDoux, J. E. (2005). Contributions of the amygdala to emotion processing: from animal models to human behavior. Neuron, 48(2), 175-187. doi:10.1016/j.neuron.2005.09.025

Phillips, M. L., Drevets, W. C., Rauch, S. L., \& Lane, R. (2003). Neurobiology of emotion perception I: The neural basis of normal emotion perception. Biol Psychiatry, 54(5), 504-514. doi:10.1016/s0006-3223(03)00168-9 
Picard, F., \& Friston, K. (2014). Predictions, perception, and a sense of self. Neurology, 83. doi:10.1212/WNL.0000000000000798

Poulet, J. F. A., \& Crochet, S. (2019). The Cortical States of Wakefulness. Frontiers in Systems Neuroscience, 12, 64-64. doi:10.3389/fnsys.2018.00064

Preller, K. H., Burt, J. B., Ji, J. L., Schleifer, C. H., Adkinson, B. D., Stämpfli, P., . . . Anticevic, A. (2018). Changes in global and thalamic brain connectivity in LSDinduced altered states of consciousness are attributable to the 5-HT2A receptor. eLife, 7, e35082. doi:10.7554/eLife.35082

Preller, K. H., Duerler, P., Burt, J. B., Ji, J. L., Adkinson, B., Stämpfli, P., . . Vollenweider, F. X. (2020). Psilocybin Induces Time-Dependent Changes in Global Functional Connectivity. Biological Psychiatry, 88(2), 197-207. doi:https://doi.org/10.1016/j.biopsych.2019.12.027

Preller, K. H., Pokorny, T., Kraehenmann, R., Scheidegger, M., Dziobek, I., Staempfli, P., \& Vollenweider, F. X. (2015). The 5-HT2A/1A agonist psilocybin reduces social pain and enhances empathy in healthy volunteers. European Neuropsychopharmacology, 25, S301-S301. doi:10.1016/s0924-977x(15)30364-3

Preller, K. H., Razi, A., Zeidman, P., Stämpfli, P., Friston, K. J., \& Vollenweider, F. X. (2019). Effective connectivity changes in LSD-induced altered states of consciousness in humans. Proceedings of the National Academy of Sciences, 116(7), 2743-2748. doi:10.1073/pnas.1815129116

Price, J., Cole, V., \& Goodwin, G. M. (2009). Emotional side-effects of selective serotonin reuptake inhibitors: qualitative study. British Journal of Psychiatry, 195(3), 211-217. doi:10.1192/bjp.bp.108.051110

Ptak, R., \& Schnider, A. (2010). The Dorsal Attention Network Mediates Orienting toward Behaviorally Relevant Stimuli in Spatial Neglect. The Journal of Neuroscience, 30(38), 12557. doi:10.1523/JNEUROSCI.2722-10.2010

Putcha, D., Ross, R. S., Cronin-Golomb, A., Janes, A. C., \& Stern, C. E. (2016). Salience and Default Mode Network Coupling Predicts Cognition in Aging and Parkinson's Disease. Journal of the International Neuropsychological Society : JINS, 22(2), 205215. doi:10.1017/S1355617715000892

Quednow, B. B., Kometer, M., Geyer, M. A., \& Vollenweider, F. X. (2012). PsilocybinInduced Deficits in Automatic and Controlled Inhibition are Attenuated by Ketanserin in Healthy Human Volunteers. Neuropsychopharmacology, 37(3), 630-640. doi:10.1038/npp.2011.228

Raichle, M. E. (2015). The brain's default mode network. Annu Rev Neurosci, 38, 433-447. doi:10.1146/annurev-neuro-071013-014030

Raichle, M. E., MacLeod, A. M., Snyder, A. Z., Powers, W. J., Gusnard, D. A., \& Shulman, G. L. (2001). A default mode of brain function. Proc Natl Acad Sci U S A, 98(2), 676682. doi:10.1073/pnas.98.2.676

Ramirez Barrantes, R., Arancibia, M., Stojanova, J., Aspé-Sánchez, M., Córdova, C., \& Henríquez, R. A. (2019). Default Mode Network, Meditation, and Age-Associated Brain Changes: What Can We Learn from the Impact of Mental Training on WellBeing as a Psychotherapeutic Approach? Neural Plasticity, 2019, 1-15. doi:10.1155/2019/7067592

Rao, R. P. N., \& Ballard, D. H. (1999). Predictive coding in the visual cortex: a functional interpretation of some extra-classical receptive-field effects. Nature Neuroscience, 2(1), 79-87. doi:10.1038/4580

Razi, A., \& Friston, K. J. (2016). The Connected Brain: Causality, models, and intrinsic dynamics. IEEE Signal Processing Magazine, 33(3), 14-35. doi:10.1109/MSP.2015.2482121 
Razi, A., Kahan, J., Rees, G., \& Friston, K. J. (2015). Construct validation of a DCM for resting state fMRI. Neuroimage, 106, 1-14. doi:10.1016/j.neuroimage.2014.11.027

Razi, A., Seghier, M. L., Zhou, Y., McColgan, P., Zeidman, P., Park, H. J., . . Friston, K. J. (2017). Large-scale DCMs for resting-state fMRI. Netw Neurosci, 1(3), 222-241. doi:10.1162/NETN_a_00015

Rens, N., Bode, S., Burianová, H., \& Cunnington, R. (2017). Proactive Recruitment of Frontoparietal and Salience Networks for Voluntary Decisions. Frontiers in Human Neuroscience, 11(610). doi:10.3389/fnhum.2017.00610

Ritchey, M., Wang, S. F., Yonelinas, A. P., \& Ranganath, C. (2019). Dissociable medial temporal pathways for encoding emotional item and context information. Neuropsychologia, 124, 66-78. doi:10.1016/j.neuropsychologia.2018.12.015

Robinson, O. J., Overstreet, C., Allen, P. S., Letkiewicz, A., Vytal, K., Pine, D. S., \& Grillon, C. (2013). The role of serotonin in the neurocircuitry of negative affective bias: serotonergic modulation of the dorsal medial prefrontal-amygdala 'aversive amplification' circuit. NeuroImage, 78, 217-223. doi:10.1016/j.neuroimage.2013.03.075

Roseman, L., Demetriou, L., Wall, M. B., Nutt, D. J., \& Carhart-Harris, R. L. (2018). Increased amygdala responses to emotional faces after psilocybin for treatmentresistant depression. Neuropharmacology, 142, 263-269. doi:https://doi.org/10.1016/j.neuropharm.2017.12.041

Roseman, L., Nutt, D. J., \& Carhart-Harris, R. L. (2018). Quality of Acute Psychedelic Experience Predicts Therapeutic Efficacy of Psilocybin for Treatment-Resistant Depression. Frontiers in Pharmacology, 8, 974. doi:10.3389/fphar.2017.00974

Roseman, L., Sereno, M., Leech, R., Kaelen, M., Orban, C., McGonigle, J., . . CarhartHarris, R. (2016). LSD alters eyes-closed functional connectivity within the early visual cortex in a retinotopic fashion. Journal of Vision, 16(12), 133-133. doi:10.1167/16.12.133

Ross, S., Bossis, A., Guss, J., Agin-Liebes, G., Malone, T., Cohen, B., . . Schmidt, B. L. (2016). Rapid and sustained symptom reduction following psilocybin treatment for anxiety and depression in patients with life-threatening cancer: a randomized controlled trial. Journal of Psychopharmacology, 30(12), 1165-1180. doi:10.1177/0269881116675512

Ruban, A., \& Kolodziej, A. A. (2018). Changes in default-mode network activity and functional connectivity as an indicator of psychedelic-assisted psychotherapy effectiveness. Neuropsychiatria I Neuropsychologia, 13(3), 91-97. doi:10.5114/nan.2018.81249

Ruck, C. A., Bigwood, J., Staples, D., Ott, J., \& Wasson, R. G. (1979). Entheogens. J Psychedelic Drugs, 11(1-2), 145-146. doi:10.1080/02791072.1979.10472098

Sampedro, F., de la Fuente Revenga, M., Valle, M., Roberto, N., Domínguez-Clavé, E., Elices, M., . . Riba, J. (2017). Assessing the Psychedelic "After-Glow" in Ayahuasca Users: Post-Acute Neurometabolic and Functional Connectivity Changes Are Associated with Enhanced Mindfulness Capacities [Formatted Article]. The International Journal of Neuropsychopharmacology.

Santos, A., Mier, D., Kirsch, P., \& Meyer-Lindenberg, A. (2011). Evidence for a general face salience signal in human amygdala. NeuroImage, 54(4), 3111-3116. doi:https://doi.org/10.1016/j.neuroimage.2010.11.024

Saulin, A., Savli, M., \& Lanzenberger, R. (2012). Serotonin and molecular neuroimaging in humans using PET. Amino Acids, 42(6), 2039-2057. doi:10.1007/s00726-011-1078-9 
Schooler, J. W., Smallwood, J., Christoff, K., Handy, T. C., Reichle, E. D., \& Sayette, M. A. (2011). Meta-awareness, perceptual decoupling and the wandering mind. Trends Cogn Sci, 15(7), 319-326. doi:10.1016/j.tics.2011.05.006

Schultze-Lutter, F., Nenadic, I., \& Grant, P. (2019). Psychosis and Schizophrenia-Spectrum Personality Disorders Require Early Detection on Different Symptom Dimensions. Frontiers in Psychiatry, 10, 476-476. doi:10.3389/fpsyt.2019.00476

Seeley, W. W., Menon, V., Schatzberg, A. F., Keller, J., Glover, G. H., Kenna, H., . . . Greicius, M. D. (2007). Dissociable intrinsic connectivity networks for salience processing and executive control. The Journal of Neuroscience, 27(9), 2349-2356. doi:10.1523/jneurosci.5587-06.2007

Seth, A. K. (2013). Interoceptive inference, emotion, and the embodied self. Trends Cogn Sci, 17(11), 565-573. doi:10.1016/j.tics.2013.09.007

Shebloski, K. L., \& Broadway, J. M. (2016). Commentary: Effects of psilocybin on time perception and temporal control of behavior in humans. Frontiers in Psychology, 7. doi:10.3389/fpsyg.2016.00736

Shin, L. M., Orr, S. P., Carson, M. A., Rauch, S. L., Macklin, M. L., Lasko, N. B., . . . Pitman, R. K. (2004). Regional cerebral blood flow in the amygdala and medial prefrontal cortex during traumatic imagery in male and female Vietnam veterans with PTSD. Arch Gen Psychiatry, 61(2), 168-176. doi:10.1001/archpsyc.61.2.168

Simon, R., \& Engström, M. (2015). The default mode network as a biomarker for monitoring the therapeutic effects of meditation. Frontiers in Psychology, 6(776). doi:10.3389/fpsyg.2015.00776

Singer, T., Critchley, H. D., \& Preuschoff, K. (2009). A common role of insula in feelings, empathy and uncertainty. Trends Cogn Sci, 13(8), 334-340. doi:10.1016/j.tics.2009.05.001

Smigielski, L., Kometer, M., Scheidegger, M., Krähenmann, R., Huber, T., \& Vollenweider, F. X. (2019). Characterization and prediction of acute and sustained response to psychedelic psilocybin in a mindfulness group retreat. Scientific Reports, 9(1), 14914. doi:10.1038/s41598-019-50612-3

Smigielski, L., Scheidegger, M., Kometer, M., \& Vollenweider, F. X. (2019). Psilocybinassisted mindfulness training modulates self-consciousness and brain default mode network connectivity with lasting effects. NeuroImage, 196, 207-215. doi:10.1016/j.neuroimage.2019.04.009

Soler, J., Franquesa, A., Feliu-Soler, A., Cebolla, A., García-Campayo, J., Tejedor, R., . . . Portella, M. J. (2014). Assessing decentering: validation, psychometric properties, and clinical usefulness of the Experiences Questionnaire in a Spanish sample. Behav Ther, 45(6), 863-871. doi:10.1016/j.beth.2014.05.004

Solms, M. L. (2018). The Neurobiological Underpinnings of Psychoanalytic Theory and Therapy. Frontiers in Behavioral Neuroscience, 12, 294-294. doi:10.3389/fnbeh.2018.00294

Speth, J., Speth, C., Kaelen, M., Schloerscheidt, A. M., Feilding, A., Nutt, D. J., \& CarhartHarris, R. L. (2016). Decreased mental time travel to the past correlates with defaultmode network disintegration under lysergic acid diethylamide. Journal of Psychopharmacology, 30(4), 344-353. doi:10.1177/0269881116628430

Spruston, N. (2008). Pyramidal neurons: dendritic structure and synaptic integration. Nat Rev Neurosci, 9(3), 206-221. doi:10.1038/nrn2286

Sridharan, D., Levitin, D. J., \& Menon, V. (2008). A critical role for the right fronto-insular cortex in switching between central-executive and default-mode networks.

Proceedings of the National Academy of Sciences, 105(34), 12569. doi:10.1073/pnas.0800005105 
Stein, T., Moritz, C., Quigley, M., Cordes, D., Haughton, V., \& Meyerand, E. (2000). Functional connectivity in the thalamus and hippocampus studied with functional MR imaging. AJNR Am J Neuroradiol, 21 (8), 1397-1401.

Steriade, M., \& Llinas, R. R. (1988). The functional states of the thalamus and the associated neuronal interplay. Physiol Rev, 68(3), 649-742. doi:10.1152/physrev.1988.68.3.649

Stevens, F. L., Hurley, R. A., Taber, K. H., Hurley, R. A., Hayman, L. A., \& Taber, K. H. (2011). Anterior Cingulate Cortex: Unique Role in Cognition and Emotion. The Journal of Neuropsychiatry and Clinical Neurosciences, 23(2), 121-125. doi:10.1176/jnp.23.2.jnp121

Strassman, R. J., Qualls, C. R., Uhlenhuth, E. H., \& Kellner, R. (1994). Dose-response study of N,N-dimethyltryptamine in humans. II. Subjective effects and preliminary results of a new rating scale. Arch Gen Psychiatry, 51(2), 98-108. doi:10.1001/archpsyc.1994.03950020022002

Studerus, E., Gamma, A., \& Vollenweider, F. X. (2010). Psychometric evaluation of the altered states of consciousness rating scale (OAV). Plos One, 5(8), e12412-e12412. doi:10.1371/journal.pone.0012412

Supekar, K., Uddin, L. Q., Prater, K., Amin, H., Greicius, M. D., \& Menon, V. (2010). Development of functional and structural connectivity within the default mode network in young children. NeuroImage, 52(1), 290-301. doi:10.1016/j.neuroimage.2010.04.009

Swanson, L. R. (2018). Unifying Theories of Psychedelic Drug Effects. Frontiers in Pharmacology, 9(172). doi:10.3389/fphar.2018.00172

Swick, D., Ashley, V., \& Turken, U. (2011). Are the neural correlates of stopping and not going identical? Quantitative meta-analysis of two response inhibition tasks. NeuroImage, 56(3), 1655-1665. doi:10.1016/j.neuroimage.2011.02.070

Tagliazucchi, E., Carhart-Harris, R., Leech, R., Nutt, D., \& Chialvo, D. R. (2014). Enhanced repertoire of brain dynamical states during the psychedelic experience. Human Brain Mapping, 35(11), 5442-5456. doi:10.1002/hbm.22562

Tagliazucchi, E., Roseman, L., Kaelen, M., Orban, C., Muthukumaraswamy, S. D., Murphy, K., . . . Carhart-Harris, R. (2016). Increased Global Functional Connectivity Correlates with LSD-Induced Ego Dissolution. Current biology: CB, 26(8), 10431050. doi:10.1016/j.cub.2016.02.010

Tavor, I., Parker Jones, O., Mars, R. B., Smith, S. M., Behrens, T. E., \& Jbabdi, S. (2016). Task-free MRI predicts individual differences in brain activity during task performance. Science (New York, N.Y.), 352(6282), 216-220. doi:10.1126/science.aad8127

Toga, A. W. (2015). Brain Mapping: An Encyclopedic Reference. 1, 1-2538.

Tompson, S., Chua, H. F., \& Kitayama, S. (2016). Connectivity between mPFC and PCC predicts post-choice attitude change: The self-referential processing hypothesis of choice justification. Hum Brain Mapp, 37(11), 3810-3820. doi:10.1002/hbm.23277

Torrico, T. J., \& Munakomi, S. (2019). Neuroanatomy, Thalamus. In StatPearls. Treasure Island (FL): StatPearls Publishing

StatPearls Publishing LLC.

Tsodyks, M., Kenet, T., Grinvald, A., \& Arieli, A. (1999). Linking spontaneous activity of single cortical neurons and the underlying functional architecture. Science, 286(5446), 1943-1946. doi:10.1126/science.286.5446.1943

Tylš, F., Palenicek, T., \& Horacek, J. (2016). Neurobiology of the Effects of Psilocybin in Relation to Its Potential Therapeutic Targets. In (pp. 782-793). 
Urgesi, C., Aglioti, S. M., Skrap, M., \& Fabbro, F. (2010). The Spiritual Brain: Selective Cortical Lesions Modulate Human Self-Transcendence. Neuron, 65(3), 309-319. doi:10.1016/j.neuron.2010.01.026

Van Essen, D. C., \& Dierker, D. L. (2007). Surface-based and probabilistic atlases of primate cerebral cortex. Neuron, 56(2), 209-225. doi:10.1016/j.neuron.2007.10.015

Vann Jones, S. A., \& O'Kelly, A. (2020). Psychedelics as a Treatment for Alzheimer's Disease Dementia. Frontiers in synaptic neuroscience, 12, 34-34. doi:10.3389/fnsyn.2020.00034

Vann, S. D., Aggleton, J. P., \& Maguire, E. A. (2009). What does the retrosplenial cortex do? Nature Reviews Neuroscience, 10(11), 792-802. doi:10.1038/nrn2733

Varela, F., Lachaux, J. P., Rodriguez, E., \& Martinerie, J. (2001). The Brainweb: Phase Synchronization and Large-Scale Integration. Nature reviews. Neuroscience, 2, 229239. doi:10.1038/35067550

Varley, T., Carhart-Harris, R., Roseman, L., Menon, D. K., \& Stamatakis, E. (2019). Serotonergic Psychedelics LSD \&amp; Psilocybin Increase the Fractal Dimension of Cortical Brain Activity in Spatial and Temporal Domains. bioRxiv, I can list detials here

, 517847. doi:10.1101/517847

Vincent, J. L., Kahn, I., Snyder, A. Z., Raichle, M. E., \& Buckner, R. L. (2008). Evidence for a frontoparietal control system revealed by intrinsic functional connectivity. $J$ Neurophysiol, 100(6), 3328-3342. doi:10.1152/jn.90355.2008

Voets, N. L., Menke, R. A. L., Jbabdi, S., Husain, M., Stacey, R., Carpenter, K., \& Adcock, J. E. (2015). Thalamo-Cortical Disruption Contributes to Short-Term Memory Deficits in Patients with Medial Temporal Lobe Damage. Cerebral Cortex, 25(11), 4584-4595. doi:10.1093/cercor/bhv109

Vogt, B. A., \& Laureys, S. (2005). Posterior cingulate, precuneal and retrosplenial cortices: cytology and components of the neural network correlates of consciousness. Prog Brain Res, 150, 205-217. doi:10.1016/s0079-6123(05)50015-3

Vogt, B. A., \& Pandya, D. N. (1987). Cingulate cortex of the rhesus monkey: II. Cortical afferents. Journal of Comparative Neurology, 262(2), 271-289. doi:https://doi.org/10.1002/cne.902620208

Vollenweider, F. X., Leenders, K. L., Scharfetter, C., Maguire, P., Stadelmann, O., \& Angst, J. (1997). Positron emission tomography and fluorodeoxyglucose studies of metabolic hyperfrontality and psychopathology in the psilocybin model of psychosis. Neuropsychopharmacology, 16(5), 357-372. doi:10.1016/s0893-133x(96)00246-1

Vollenweider, F. X., \& Preller, K. H. (2020). Psychedelic drugs: neurobiology and potential for treatment of psychiatric disorders. Nature Reviews Neuroscience, 21(11), 611-624. doi:10.1038/s41583-020-0367-2

Vollenweider, F. X., Vollenweider-Scherpenhuyzen, M. F. I., Babler, A., Vogel, H., \& Hell, D. (1998). Psilocybin induces schizophrenia-like psychosis in humans via a serotonin2 agonist action. Neuroreport, 9(17), 3897-3902. doi:10.1097/00001756-19981201000024

Wagner, M. T., Mithoefer, M. C., Mithoefer, A. T., MacAulay, R. K., Jerome, L., YazarKlosinski, B., \& Doblin, R. (2017). Therapeutic effect of increased openness: Investigating mechanism of action in MDMA-assisted psychotherapy. $J$ Psychopharmacol, 31(8), 967-974. doi:10.1177/0269881117711712

Ward, A. M., Schultz, A. P., Huijbers, W., Van Dijk, K. R., Hedden, T., \& Sperling, R. A. (2014). The parahippocampal gyrus links the default-mode cortical network with the medial temporal lobe memory system. Hum Brain Mapp, 35(3), 1061-1073. doi:10.1002/hbm.22234 
Wasson, G. R., Hofmann, Albert., Ruck Carl A.P. (1978). The Road to Eleusis: Unveiling the Secret of the Mysteries. New York: Harcourt Brace Jovanovich, Inc.

Watts, R., Day, C., Krzanowski, J., Nutt, D., \& Carhart-Harris, R. (2017). Patients' Accounts of Increased "Connectedness" and "Acceptance" After Psilocybin for TreatmentResistant Depression. Journal of Humanistic Psychology, 57(5), 520-564. doi:10.1177/0022167817709585

Weniger, G., Lange, C., Sachsse, U., \& Irle, E. (2009). Reduced amygdala and hippocampus size in trauma-exposed women with borderline personality disorder and without posttraumatic stress disorder. J Psychiatry Neurosci, 34(5), 383-388.

Whitfield-Gabrieli, S., \& Ford, J. M. (2012). Default mode network activity and connectivity in psychopathology. Annu Rev Clin Psychol, 8, 49-76. doi:10.1146/annurev-clinpsy032511-143049

Whitfield-Gabrieli, S., Thermenos, H. W., Milanovic, S., Tsuang, M. T., Faraone, S. V., McCarley, R. W., . . Seidman, L. J. (2009). Hyperactivity and hyperconnectivity of the default network in schizophrenia and in first-degree relatives of persons with schizophrenia. Proc Natl Acad Sci U S A, 106(4), 1279-1284. doi:10.1073/pnas.0809141106

Winkelman, M., \& Hoffman, M. (2015). Hallucinogens and Entheogens. In (pp. vol 2: 126132).

Wise, T., Marwood, L., Perkins, A. M., Herane-Vives, A., Joules, R., Lythgoe, D. J., . . Arnone, D. (2017). Instability of default mode network connectivity in major depression: a two-sample confirmation study. Translational psychiatry, 7(4), e1105e1105. doi:10.1038/tp.2017.40

Wolff, M., Evens, R., Mertens, L. J., Koslowski, M., Betzler, F., Gründer, G., \& Jungaberle, H. (2020). Learning to Let Go: A Cognitive-Behavioral Model of How Psychedelic Therapy Promotes Acceptance. Frontiers in Psychiatry, 11, 5-5. doi:10.3389/fpsyt.2020.00005

Wolland, A. M., \& Hagelsteen, J. H. (1991). [Prosopagnosia. A rare disorder of visual perception]. Tidsskr Nor Laegeforen, 111(29), 3505-3506.

Yaden, D. B., \& Griffiths, R. R. (2020). The Subjective Effects of Psychedelics Are Necessary for Their Enduring Therapeutic Effects. ACS Pharmacology \& Translational Science. doi:10.1021/acsptsci.0c00194

Zamaria, J. A. (2016). A Phenomenological Examination of Psilocybin and its Positive and Persisting Aftereffects. Neuroquantology, 14(2), 285-296. doi:10.14704/nq.2016.14.2.943

Zeki, S. (1991). Cerebral akinetopsia (visual motion blindness). A review. Brain, 114 ( Pt 2), 811-824. doi:10.1093/brain/114.2.811

Zeki, S. (2005). The Ferrier Lecture 1995 behind the seen: The functional specialization of the brain in space and time. Philos. Trans. R. Soc. Lond. B. Biol. Sci., 360(1458), $1145-1183$.

Zeki, S., \& Shipp, S. (1988). The functional logic of cortical connections. Nature, 335, 311317.

Zeng, L.-L., Shen, H., Liu, L., Wang, L., Li, B., Fang, P., . . . Hu, D. (2012). Identifying major depression using whole-brain functional connectivity: a multivariate pattern analysis. Brain, 135(5), 1498-1507. doi:10.1093/brain/aws059

Zhang, R., \& Volkow, N. D. (2019). Brain default-mode network dysfunction in addiction. NeuroImage, 200, 313-331. doi:https://doi.org/10.1016/j.neuroimage.2019.06.036

Zhao, X.-H., Wang, P.-J., Li, C.-B., Hu, Z.-H., Xi, Q., Wu, W.-Y., \& Tang, X.-W. (2007). Altered default mode network activity in patient with anxiety disorders: An fMRI 
study. European Journal of Radiology, 63(3), 373-378.

doi:https://doi.org/10.1016/j.ejrad.2007.02.006

Zhou, Y., Friston, K. J., Zeidman, P., Chen, J., Li, S., \& Razi, A. (2018). The Hierarchical Organization of the Default, Dorsal Attention and Salience Networks in Adolescents and Young Adults. Cereb Cortex, 28(2), 726-737. doi:10.1093/cercor/bhx307 